\title{
THE MOST METAL-POOR STARS. II. CHEMICAL ABUNDANCES OF 190 METAL-POOR STARS INCLUDING 10 NEW STARS WITH $[\mathrm{Fe} / \mathrm{H}] \leqslant-3.5^{*, \dagger, \sharp}$
}

\author{
David Yong ${ }^{1}$, John E. Norris ${ }^{1}$, M. S. Bessell ${ }^{1}$, N. Christlieb ${ }^{2}$, M. Asplund ${ }^{1,3}$, Timothy C. Beers ${ }^{4,5}$, \\ P. S. BARKLEM ${ }^{6}$, ANNA Frebel ${ }^{7}$, AND S. G. Ryan ${ }^{8}$ \\ ${ }^{1}$ Research School of Astronomy and Astrophysics, The Australian National University, Weston, ACT 2611, Australia; \\ yong@mso.anu.edu.au, jen@mso.anu.edu.au, bessell@mso.anu.edu.au, martin@mso.anu.edu.au \\ 2 Zentrum für Astronomie der Universität Heidelberg, Landessternwarte, Königstuhl 12, \\ D-69117 Heidelberg, Germany; n.christlieb@1sw.uni-heidelberg.de \\ ${ }^{3}$ Max-Planck Institute for Astrophysics, Karl-Schwarzschild Str. 1, D-85741 Garching, Germany \\ ${ }^{4}$ National Optical Astronomy Observatory, Tucson, AZ 85719, USA \\ ${ }^{5}$ Department of Physics \& Astronomy and JINA (Joint Institute for Nuclear Astrophysics), \\ Michigan State University, East Lansing, MI 48824, USA; beers@pa.msu.edu \\ ${ }^{6}$ Department of Physics and Astronomy, Uppsala University, Box 515, SE-75120 Uppsala, Sweden; paul.barklem@physics.uu.se \\ ${ }^{7}$ Massachusetts Institute of Technology, Kavli Institute for Astrophysics and Space Research, Cambridge, MA 02139, USA; afrebel@mit.edu \\ ${ }^{8}$ Centre for Astrophysics Research, School of Physics, Astronomy \& Mathematics, University of Hertfordshire, \\ College Lane, Hatfield, Hertfordshire AL10 9AB, UK; s.g.ryan@ herts.ac.uk \\ Received 2012 April 12; accepted 2012 July 19; published 2012 December 13
}

\begin{abstract}
We present a homogeneous chemical abundance analysis of 16 elements in 190 metal-poor Galactic halo stars (38 program and 152 literature objects). The sample includes 171 stars with $[\mathrm{Fe} / \mathrm{H}] \leqslant-2.5$, of which 86 are extremely metal poor, $[\mathrm{Fe} / \mathrm{H}] \leqslant-3.0$. Our program stars include 10 new objects with $[\mathrm{Fe} / \mathrm{H}] \leqslant-3.5$. We identify a sample of "normal" metal-poor stars and measure the trends between $[\mathrm{X} / \mathrm{Fe}]$ and $[\mathrm{Fe} / \mathrm{H}]$, as well as the dispersion about the mean trend for this sample. Using this mean trend, we identify objects that are chemically peculiar relative to "normal" stars at the same metallicity. These chemically unusual stars include CEMP-no objects, one star with high $[\mathrm{Si} / \mathrm{Fe}]$, another with high $[\mathrm{Ba} / \mathrm{Sr}]$, and one with unusually low $[\mathrm{X} / \mathrm{Fe}]$ for all elements heavier than $\mathrm{Na}$. The $\mathrm{Sr}$ and $\mathrm{Ba}$ abundances indicate that there may be two nucleosynthetic processes at lowest metallicity that are distinct from the main $r$-process. Finally, for many elements, we find a significant trend between $[\mathrm{X} / \mathrm{Fe}]$ versus $T_{\text {eff }}$, which likely reflects non-LTE and/or three-dimensional effects. Such trends demonstrate that care must be exercised when using abundance measurements in metal-poor stars to constrain chemical evolution and/or nucleosynthesis predictions.
\end{abstract}

Key words: early universe - Galaxy: formation - Galaxy: halo - nuclear reactions, nucleosynthesis, abundances stars: abundances

Online-only material: color figures, machine-readable tables

\section{INTRODUCTION}

The atmospheres of low-mass stars contain detailed information on the chemical composition of the interstellar medium at the time and place of their birth. Thus, studies of the most metal-poor stars of the Galactic halo arguably offer the best means with which to understand the properties of the first stars (e.g., Chamberlain \& Aller 1951; Wallerstein et al. 1963; Carney \& Peterson 1981; Bessell \& Norris 1984; McWilliam et al. 1995; Ryan et al. 1996; Norris et al. 2001; Johnson 2002; Cayrel et al. 2004; Beers \& Christlieb 2005; Cohen et al. 2008; Lai et al. 2008; Frebel \& Norris 2011). In recent times, four stars with an iron content less than $\sim 1 / 30,000$ that of the Sun have been discovered-HE 0107-5240 (Christlieb et al. 2002, 2004), HE 1327-2326 (Frebel et al. 2005; Aoki et al. 2006), and HE 0557-4840 (Norris et al. 2007), within the Hamburg/ESO

\footnotetext{
* This paper includes data gathered with the $6.5 \mathrm{~m}$ Magellan Telescopes located at Las Campanas Observatory, Chile.

$\dagger$ Some of the data presented herein were obtained at the W. M. Keck

Observatory, which is operated as a scientific partnership among the California Institute of Technology, the University of California, and the National

Aeronautics and Space Administration. The Observatory was made possible

by the generous financial support of the W. M. Keck Foundation.

$¥$ Based on observations collected at the European Organisation for

Astronomical Research in the Southern Hemisphere, Chile (proposal

281.D-5015).
}

Survey (HES; Wisotzki et al. 1996), and SDSS J102915+172927 (Caffau et al. 2011), in the Sloan Digital Sky Survey Data Release 7 (Abazajian et al. 2009). The abundance patterns of these stars can constrain yields from zero-metallicity supernovae (e.g., Limongi et al. 2003; Umeda \& Nomoto 2005; Meynet et al. 2006; Tominaga et al. 2007; Heger \& Woosley 2010; Limongi \& Chieffi 2012).

Chemical abundance studies of metal-poor stars with increasing accuracy and precision have, in some cases, revealed extremely small scatter in abundance ratios at low metallicity (Cayrel et al. 2004; Arnone et al. 2005). Such results place strong constraints on the yields of the progenitor stars, as well as on the relative contributions of intrinsic scatter and measurement errors. Meanwhile, parallel studies have started to identify a variety of chemically diverse objects (e.g., Aoki et al. 2005; Cohen et al. 2007; Lai et al. 2009), which may indicate that "we are beginning to see the anticipated and long sought stochastic effects of individual supernova events contributing to the Fepeak material within a single star" (Cohen et al. 2008, abstract).

As the numbers of metal-poor stars with detailed chemical abundance measurements have grown, databases have been compiled (Suda et al. 2008, 2011; Frebel 2010) that facilitate studies of the global characteristics of metal-poor stars (see also Roederer 2009, who assembled and studied a compilation of nearly 700 halo stars). However, it is difficult, perhaps 
impossible, to understand whether the trends and dispersions in $[\mathrm{X} / \mathrm{Fe}]^{9}$ versus $[\mathrm{Fe} / \mathrm{H}]$ are real, or an artifact of an inhomogeneous comparison. Moreover, in the absence of a careful, homogeneous analysis, subtle effects may be overlooked.

In order to make further progress in this field, there is a clear need for a detailed homogeneous chemical abundance analysis of a large sample of metal-poor stars. To this end, Barklem et al. (2005) studied 253 stars, presenting chemical abundance measurements for some 22 elements. Their study included 49 new stars with $[\mathrm{Fe} / \mathrm{H}]<-3$, but only one of which had $[\mathrm{Fe} / \mathrm{H}] \leqslant-3.5$, thereby highlighting the difficulty of finding stars in this metallicity regime.

This is the second paper in our series on the discovery and analysis of the most metal-poor stars. Here, we present a homogeneous chemical abundance analysis for 38 program stars and a further 152 literature stars. Our program stars include 10 new objects with $[\mathrm{Fe} / \mathrm{H}] \leqslant-3.5$, and the combined sample includes 86 extremely metal-poor stars, $[\mathrm{Fe} / \mathrm{H}] \leqslant-3.0$. To our knowledge, this represents one of the largest homogeneous chemical abundance analyses of the most metal-poor stars to date based on model atmosphere analysis of equivalent widths measured in high-resolution, high signal-to-noise ratio $(\mathrm{S} / \mathrm{N})$ spectra. The outline of the paper is as follows. In Section 2, we describe the analysis of the 38 program stars. In Section 3, we compare our chemical abundances with those of the FIRST STARS group (Cayrel et al. 2004; Spite et al. 2005; François et al. 2007). In Section 4, we describe our homogeneous re-analysis of 207 literature stars; in Section 5, we compare these results with the literature values. In Section 6, we consider non-LTE effects. Finally, our results, interpretation, and conclusions are presented in Sections 7 and 8.

\section{OBSERVATIONS AND ANALYSIS OF 38 PROGRAM STARS}

\subsection{Stellar Parameters: $T_{\text {eff }}, \operatorname{logg}$, $\xi_{t}$, and $[\mathrm{Fe} / \mathrm{H}]$}

In Norris et al. (2013a; Paper I) we describe high-resolution spectroscopic observations of 38 program stars (obtained using the Keck, Magellan, and VLT telescopes), including the discovery and sample selection, equivalent-width measurements, radial velocities, and line list. Our sample comprises 34 stars original to the present work, together with four for which published abundances already exist, or which are the subject of analyses currently underway. In Paper I, we also describe the temperature scale, which consists of spectrophotometry and Balmer-line analysis. We refer the reader to these works for the details of the observational data upon which the present analysis is based.

With effective temperatures, $T_{\text {eff }}$, and equivalent widths in hand, our analysis proceeded in the following manner. Surface gravities, ${ }^{10} \log g$, were taken from the $Y^{2}$ isochrones (Demarque et al. 2004), assuming an age of $10 \mathrm{Gyr}$ and $[\alpha / \mathrm{Fe}]=+0.3$. We note that changing the age from $10 \mathrm{Gyr}$ to $13 \mathrm{Gyr}$ (or $7 \mathrm{Gyr}$ ) would only introduce a systematic difference in $\log g$ of $\leqslant 0.1 \mathrm{dex}$. We also note that these isochrones only extend down

\footnotetext{
9 We adopt the usual spectroscopic notations that $[A / B] \equiv \log _{10}\left(N_{A} / N_{B}\right)_{\star}$ $\log _{10}\left(N_{A} / N_{B}\right)_{\odot}$, and that $\log \varepsilon(B)=A(B) \equiv \log _{10}\left(N_{B} / N_{\mathrm{H}}\right)+12.00$, for elements $A$ and $B$.

10 For 29 program stars, the spectrophotometric and Balmer-line analyses provided agreement on the evolutionary status: dwarf, subgiant, giant, or horizontal branch. For the nine remaining program stars, there was disagreement between spectrophotometric and Balmer-line analyses on the evolutionary status: dwarf vs. subgiant in all cases. We therefore conducted two analyses of each of these nine stars, one assuming a "dwarf" gravity and the other assuming a "subgiant" gravity.
}

to $[\mathrm{Fe} / \mathrm{H}]=-3.5$; therefore, the surface gravity we obtain for more metal-poor stars involves a linear extrapolation, from $[\mathrm{Fe} / \mathrm{H}]=-3.5$ down to $[\mathrm{Fe} / \mathrm{H}]=-4.1$, for the most metalpoor stars in our program sample. For our four most metal-poor stars, we note that the average difference between the surface gravity inferred using $[\mathrm{Fe} / \mathrm{H}]=-3.5$ (the boundary value of the $Y^{2}$ isochrones) and the extrapolated surface gravity using the actual $[\mathrm{Fe} / \mathrm{H}]$ is $0.06 \mathrm{dex}$ (for the giant/subgiant case) and 0.01 dex (for the dwarf case). Initial estimates of the metallicity came from the medium-resolution follow-up spectroscopy.

Model atmospheres were taken from the $\alpha$-enhanced, $[\alpha / \mathrm{Fe}]=+0.4$, NEWODF grid of ATLAS9 models by Castelli \& Kurucz (2003). These one-dimensional (1D), plane-parallel, local thermodynamic equilibrium (LTE) models were computed using a microturbulent velocity of $2 \mathrm{~km} \mathrm{~s}^{-1}$ and no convective overshooting. Interpolation within the grid was necessary to produce models with the required combination of $T_{\text {eff }}, \log g$, and $[\mathrm{M} / \mathrm{H}]$. The interpolation software, kindly provided by Dr. Carlos Allende Prieto, has been used extensively (e.g., Reddy et al. 2003; Allende Prieto et al. 2004), and involves linear interpolation in three dimensions $\left(T_{\text {eff }}, \log g\right.$, and $\left.[\mathrm{M} / \mathrm{H}]\right)$ to produce the required model.

The final tool in our analysis kit was the LTE stellar lineanalysis program MOOG (Sneden 1973). The particular version of MOOG that we used includes a proper treatment of continuum scattering (see Sobeck et al. 2011 for further details). We refer the reader to Cayrel et al. (2004) and Sobeck et al. (2011) for a discussion regarding the importance of Raleigh scattering (Griffin et al. 1982) at blue wavelengths in metal-poor stars.

Having computed the abundance for each line, the microturbulent velocity was determined, in the usual way, by forcing the abundances from Fe I lines to have no trend with the reduced equivalent width, $\log \left(W_{\lambda} / \lambda\right)$. The metallicity, $[\mathrm{Fe} / \mathrm{H}]$, was inferred exclusively from Fe I lines. While we are mindful that such lines are more susceptible to non-LTE effects than Fe II lines (e.g., Asplund 2005), we were unable to measure any Fe II lines for a number of program stars. Higher-quality spectra are necessary to measure additional Fe II lines in our sample. In Section 6, we shall compare iron abundances derived from neutral and ionized species for those stars for which data are available.

With an updated estimate of the metallicity, $[\mathrm{Fe} / \mathrm{H}]_{\text {star }}$, we then compared this value with the metallicity assumed when generating the model atmosphere, $[\mathrm{M} / \mathrm{H}]_{\text {model }}$. If the difference exceeded 0.1 dex, we computed an updated model atmosphere with $[\mathrm{M} / \mathrm{H}]_{\text {new }}=[\mathrm{Fe} / \mathrm{H}]_{\text {star }}$. Based on the new metallicity, the surface gravity was revised and the star was re-analyzed. When this was required, we note that the abundance from Fe I lines and the metallicity of the model atmosphere converged within one or two iterations. That is, the inferred abundance, $[\mathrm{Fe} / \mathrm{H}]$, is only weakly dependent on the metallicity of the model, $[\mathrm{M} / \mathrm{H}]$, provided the initial guess is close to the final value. During the analysis process, we removed $\mathrm{Fe}$ I lines for which the abundance differed from (1) the median abundance by more than $0.5 \mathrm{dex}$ or (2) the median abundance by more than $3 \sigma$. (Lines yielding abundances higher or lower than the median value by more than 0.5 dex or $3 \sigma$ were rejected.) This criterion resulted in the rejection of a handful of lines for a given star. The largest number of rejected lines for a given star was 6, leaving 33 accepted lines, while the largest fraction of rejected lines was 3 , leaving 15 accepted lines.

In the course of our analysis, another consideration was whether or not a given Fe line might be blended with $\mathrm{CH}$ 
Table 1

Model Atmosphere Parameters and $[\mathrm{Fe} / \mathrm{H}]$ for the 38 Program Stars

\begin{tabular}{|c|c|c|c|c|c|c|c|c|}
\hline Star & $\begin{array}{c}\text { RA2000 } \\
\text { (2) }\end{array}$ & $\begin{array}{c}\mathrm{DEC}_{2000^{\mathrm{a}}} \\
\text { (3) }\end{array}$ & $\begin{array}{l}T_{\text {eff }} \\
(\mathrm{K}) \\
(4)\end{array}$ & $\begin{array}{c}\log g \\
(\mathrm{cgs}) \\
(5)\end{array}$ & $\begin{array}{c}\xi_{t} \\
\left(\mathrm{~km} \mathrm{~s}^{-1}\right) \\
(6)\end{array}$ & $\begin{array}{c}{[\mathrm{M} / \mathrm{H}]_{\text {model }}} \\
\text { (7) }\end{array}$ & $\begin{array}{c}{[\mathrm{Fe} / \mathrm{H}]_{\text {derived }}} \\
(8)\end{array}$ & $\begin{array}{c}\text { C-rich }{ }^{\mathrm{b}} \\
(9)\end{array}$ \\
\hline 52972-1213-507 & 091849.9 & +374426.8 & 6463 & 4.34 & 1.2 & -3.0 & -2.98 & 1 \\
\hline $53327-2044-515^{\mathrm{c}}$ & 014036.2 & +234458.1 & 5703 & 4.68 & 0.8 & -4.0 & -4.00 & 1 \\
\hline $53327-2044-515^{d}$ & 014036.2 & +234458.1 & 5703 & 3.36 & 1.5 & -4.1 & -4.09 & 1 \\
\hline 53436-1996-093 & 112813.6 & +384148.9 & 6449 & 4.38 & 1.3 & -3.5 & -3.53 & 0 \\
\hline 54142-2667-094 & 085136.7 & +101803.2 & 6456 & 3.87 & 1.4 & -3.0 & -2.96 & 0 \\
\hline BS $16545-089$ & 112427.5 & +365028.8 & 6486 & 3.82 & 1.4 & -3.4 & -3.44 & 0 \\
\hline CS 30336-049 & 204523.5 & -284235.9 & 4725 & 1.19 & 2.1 & -4.1 & -4.10 & 0 \\
\hline HE 0049-3948 & 005213.4 & -393236.9 & 6466 & 3.78 & 0.8 & -3.7 & -3.68 & 0 \\
\hline HE 0057-5959 & 005954.0 & -594329.9 & 5257 & 2.65 & 1.5 & -4.1 & -4.08 & 1 \\
\hline HE $0102-1213$ & 010528.0 & -115731.1 & 6100 & 3.65 & 1.5 & -3.3 & -3.28 & 0 \\
\hline HE $0146-1548$ & 014834.7 & -153324.4 & 4636 & 0.99 & 2.1 & -3.5 & -3.46 & 1 \\
\hline HE $0207-1423$ & 021000.7 & -140911.1 & 5023 & 2.07 & 1.3 & -3.0 & -2.95 & 1 \\
\hline HE $0228-4047^{c}$ & 023033.7 & -403354.8 & 6515 & 4.35 & 1.6 & -3.8 & -3.75 & 0 \\
\hline HE $0228-4047^{\mathrm{d}}$ & 023033.7 & -403354.8 & 6515 & 3.80 & 1.7 & -3.8 & -3.75 & 0 \\
\hline HE $0231-6025$ & 023230.6 & -601211.2 & 6437 & 4.36 & 1.8 & -3.1 & -3.10 & 0 \\
\hline HE $0253-1331$ & 025606.7 & -131927.0 & 6474 & 4.34 & 1.5 & -3.0 & -3.01 & 0 \\
\hline HE 0314-1739 & 031701.8 & -172854.9 & 6570 & 4.25 & 1.1 & -2.9 & -2.86 & 0 \\
\hline HE $0355-3728^{c}$ & 035636.5 & -443403.4 & 6418 & 4.39 & 1.4 & -3.4 & -3.41 & 0 \\
\hline HE $0355-3728^{d}$ & 035636.5 & -443403.4 & 6418 & 3.84 & 1.5 & -3.4 & -3.41 & 0 \\
\hline HE $0945-1435^{\mathrm{c}}$ & 094750.7 & -144906.9 & 6344 & 4.43 & 1.2 & -3.8 & -3.77 & 0 \\
\hline HE $0945-1435^{\mathrm{d}}$ & 094750.7 & -144906.9 & 6344 & 3.71 & 1.4 & -3.8 & -3.78 & 0 \\
\hline $\mathrm{HE} 1055+0104^{\mathrm{c}}$ & 105804.4 & +004836.0 & 6287 & 4.43 & 1.3 & -2.9 & -2.87 & 0 \\
\hline HE $1055+0104^{d}$ & 105804.4 & +004836.0 & 6287 & 3.79 & 1.5 & -2.9 & -2.89 & 0 \\
\hline HE $1116-0054^{c}$ & 111847.8 & -011119.4 & 6454 & 4.37 & 1.6 & -3.5 & -3.49 & 0 \\
\hline HE $1116-0054^{\mathrm{d}}$ & 111847.8 & -011119.4 & 6454 & 3.80 & 1.6 & -3.5 & -3.48 & 0 \\
\hline HE $1142-1422$ & 114459.2 & -143849.6 & 6238 & 2.80 & 3.4 & -2.8 & -2.84 & 0 \\
\hline HE $1201-1512^{c}$ & 120337.0 & -152933.0 & 5725 & 4.67 & 0.9 & -3.9 & -3.86 & 1 \\
\hline HE $1201-1512^{d}$ & 120337.0 & -152933.0 & 5725 & 3.39 & 1.5 & -3.9 & -3.92 & 1 \\
\hline HE 1204-0744 & 120646.2 & -080044.1 & 6500 & 4.30 & 1.8 & -2.7 & -2.71 & 0 \\
\hline HE $1207-3108$ & 120954.0 & -312510.6 & 5294 & 2.85 & 0.9 & -2.7 & -2.70 & 0 \\
\hline HE $1320-2952$ & 132254.9 & -300805.3 & 5106 & 2.26 & 1.5 & -3.7 & -3.69 & 0 \\
\hline HE $1346-0427^{\mathrm{c}}$ & 134925.1 & -044214.8 & 6255 & 4.47 & 1.2 & -3.6 & -3.57 & 0 \\
\hline HE $1346-0427^{d}$ & 134925.1 & -044214.8 & 6255 & 3.69 & 1.4 & -3.6 & -3.58 & 0 \\
\hline $\mathrm{HE} 1402-0523^{\mathrm{c}}$ & 140438.0 & -053813.5 & 6418 & 4.38 & 1.0 & -3.2 & -3.17 & 0 \\
\hline HE $1402-0523^{\mathrm{d}}$ & 140438.0 & -053813.5 & 6418 & 3.82 & 1.2 & -3.2 & -3.19 & 0 \\
\hline HE $1506-0113$ & 150914.3 & -012456.6 & 5016 & 2.01 & 1.6 & -3.5 & -3.54 & 1 \\
\hline HE $2020-5228$ & 202417.1 & -521902.3 & 6305 & 3.79 & 1.4 & -2.9 & -2.93 & 0 \\
\hline HE $2032-5633$ & 203624.9 & -562305.8 & 6457 & 3.78 & 1.8 & -3.6 & -3.63 & 0 \\
\hline HE $2047-5612$ & 205122.1 & -560052.9 & 6128 & 3.68 & 0.9 & -3.1 & -3.14 & 0 \\
\hline HE $2135-1924$ & 213804.7 & -191104.4 & 6449 & 4.37 & 1.2 & -3.3 & -3.31 & 0 \\
\hline HE $2136-6030$ & 214039.5 & -601626.4 & 6409 & 3.85 & 2.0 & -2.9 & -2.88 & 0 \\
\hline HE $2139-5432$ & 214242.4 & -541842.9 & 5416 & 3.04 & 0.8 & -4.0 & -4.02 & 1 \\
\hline HE $2141-0726$ & 214406.6 & -071248.9 & 6551 & 4.26 & 1.5 & -2.7 & -2.72 & 0 \\
\hline HE $2142-5656$ & 214620.4 & -564219.1 & 4939 & 1.85 & 2.1 & -2.9 & -2.87 & 1 \\
\hline HE $2202-4831$ & 220605.8 & -481653.0 & 5331 & 2.95 & 1.2 & -2.8 & -2.78 & 1 \\
\hline HE $2246-2410$ & 224859.6 & -235439.0 & 6431 & 4.36 & 1.5 & -3.0 & -2.96 & 0 \\
\hline HE $2247-7400$ & 225119.4 & -734423.6 & 4829 & 1.56 & 2.0 & -2.9 & -2.87 & 1 \\
\hline
\end{tabular}

Notes.

${ }^{\text {a }}$ Coordinates are from the 2MASS database (Skrutskie et al. 2006).

b $1=$ CEMP object, adopting the Aoki et al. (2007) definition and $0=$ C-normal (see Section 7.1 for details).

${ }^{\mathrm{c}}$ For this set of results, a dwarf gravity is assumed (see Section 2.1 for details).

${ }^{\mathrm{d}}$ For this set of results, a subgiant gravity is assumed (see Section 2.1 for details).

molecular lines. Therefore, we repeated the entire analysis using a subset of lines which spectrum synthesis suggests are not blended with $\mathrm{CH}$ (see Norris et al. 1997, 2010a for further details). For the microturbulent velocity and metallicity, the results from the two approaches are very similar. Once we had measured the $[\mathrm{C} / \mathrm{Fe}]$ abundance ratio, we adopted the results using the $\mathrm{CH}$-free line list if the program star was a carbonenhanced metal-poor (CEMP) object (we applied the Aoki et al.
2007 CEMP definition). Details on the $\mathrm{C}$ measurements and CEMP definition are provided in Sections 2.2 and 7.1. In Table 1, we present the stellar parameters for the program stars. The evolutionary status, $T_{\text {eff }}$ versus $\log g$, for the program stars is shown in Figure 1.

We note here that for the nine stars for which we conducted separate analyses using a dwarf gravity and a subgiant gravity, the average difference in iron abundance between the dwarf and 


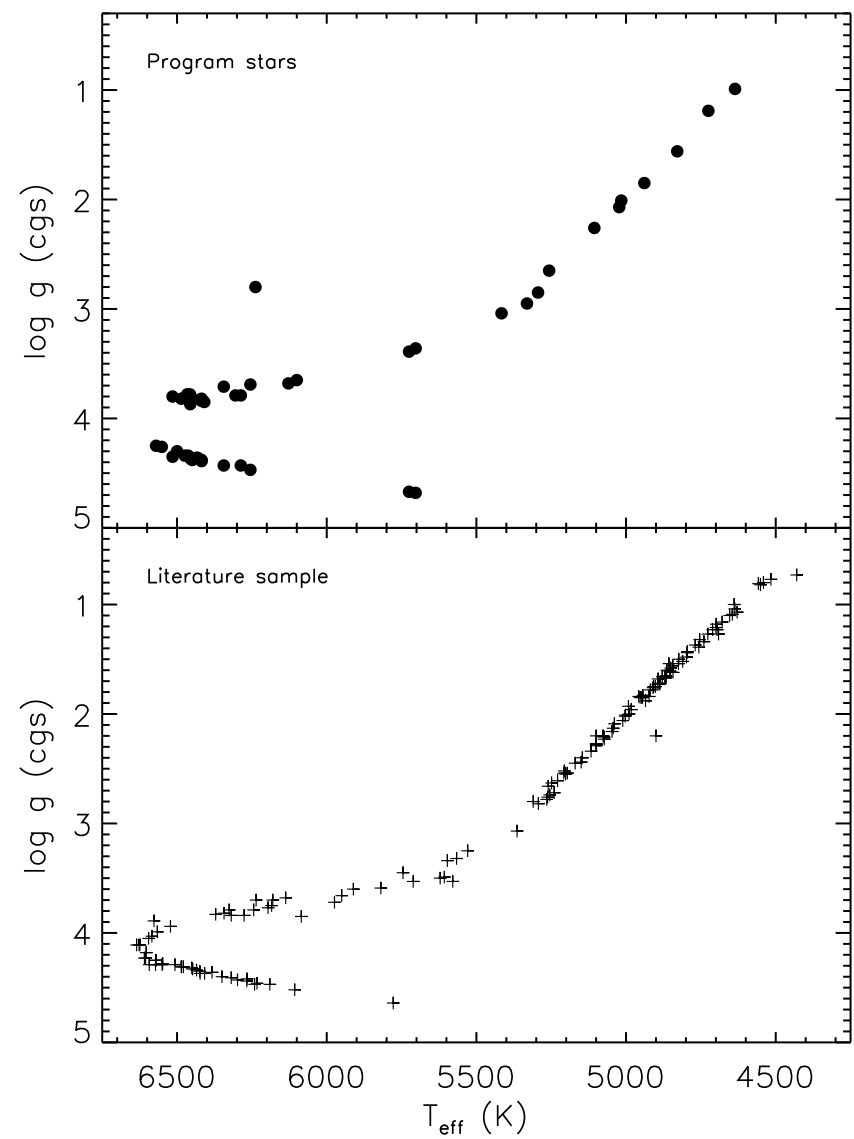

Figure 1. $T_{\text {eff }}$ vs. $\log g$ for our sample (upper panel) and for the literature sample (lower panel). Note the location of the horizontal-branch star (HE 1142-1422) in the upper panel.

subgiant analyses is $\left\langle[\mathrm{Fe} / \mathrm{H}]_{\text {dwarf }}-[\mathrm{Fe} / \mathrm{H}]_{\text {subgiant }}\right\rangle=0.02 \pm$ 0.01 dex $(\sigma=0.03)$; the relative abundance is $\left\langle[\mathrm{X} / \mathrm{Fe}]_{\text {dwarf }}-\right.$ $\left.[\mathrm{X} / \mathrm{Fe}]_{\text {subgiant }}\right\rangle=0.05 \pm 0.02 \mathrm{dex}(\sigma=0.16)$. Given these modest differences, for a given star we average the abundance ratios $[\mathrm{Fe} / \mathrm{H}]$ and $[\mathrm{X} / \mathrm{Fe}]$ for the dwarf and subgiant cases. Unless noted otherwise, we use these average values for a given star throughout the remainder of the paper. We report the values for the individual analyses in the relevant tables.

\subsection{Element Abundances}

The abundances of atomic lines were computed using our measured equivalent widths and $\log g f$ values from Paper I, final model atmospheres, and MOOG. Lines of Sc II, Mn I, Co I, and $\mathrm{Ba}$ II are affected by hyperfine splitting. In our analysis, we treated these lines appropriately, using the data from Kurucz \& Bell (1995). Additionally, Ba II lines are affected by isotopic splitting. Our $\mathrm{Ba}$ abundances were computed assuming the McWilliam (1998) $r$-process isotopic composition and hyperfine splitting. For a restricted number of elements in some stars, we determined upper limits to the chemical abundance, based on equivalent-width limits presented in Paper I.

Given the low metallicities of our sample and the $\mathrm{S} / \mathrm{N}$ of our spectra, we are not well positioned to measure the abundance of oxygen. That said, we have determined the abundance (or its upper limit) of this element for six C-rich (i.e., CEMP) stars in our sample, which we shall discuss further in Paper IV (Norris et al. 2013b). For HE 2139-5432, the oxygen abundance was derived from analysis of the $7771.94 \AA$,
$7774.17 \AA$, and $7775.39 \AA$ lines. The measured equivalent widths for these lines are $20.1 \mathrm{~m} \AA, 18.7 \mathrm{~m} \AA$, and $12.2 \mathrm{~m} \AA$ and the adopted $\log g f$ values are $0.324,0.174$, and -0.046 , respectively. Thus, we obtained an LTE abundance of $A(\mathrm{O})=$ $7.82(\sigma=0.05)$ for HE 2139-5432, which corresponds to $[\mathrm{O} / \mathrm{Fe}]=+3.15$ (we adopt the Asplund et al. 2009 solar abundances). For HE 0146-1548 and HE 1506-0113, the O limits $([\mathrm{O} / \mathrm{Fe}]<+1.63$ and +2.32 , respectively) were determined using an equivalent width of $<10 \mathrm{~m} \AA$ for the $6300.30 \AA$ line, adopting $\log g f=-9.820$. Finally, for 53327-2044-515, HE 0057-5959, and HE 1201-1512, the O limits ([O/Fe] < $+2.81,+2.77$, and +2.64 , respectively) were determined using an equivalent width of $10 \mathrm{~m} \AA$ for the $7771.94 \AA$ line (where for 53327-2044-515 and HE 1201-1512 the abundance value for each star is the mean of the low- and high-gravity solutions).

For $\mathrm{C}$ and $\mathrm{N}$, abundances (or upper limits) were determined from analysis of the $(0-0)$ and $(1-1)$ bands of the $A-X$ electronic transitions of the $\mathrm{CH}$ molecule (4290 $\AA$ to $4330 \AA$ ) and the NH molecule ( $3350 \AA$ to $3370 \AA$ ). We compared synthetic spectra, generated using MOOG, with the observed spectra and adjusted the input abundance until the two spectra matched. The macroturbulent broadening was determined using a Gaussian representing the combined effects of the instrumental profile, atmospheric turbulence, and stellar rotation. The width of this Gaussian was estimated during the course of the spectrum synthesis fitting, and the $\mathrm{C}$ and $\mathrm{N}$ abundances are thus (slightly) sensitive to the adopted broadening. Following the analysis described in Norris et al. (2010b), the $\mathrm{CH}$ line list was that compiled by B. Plez et al. (2009, private communication). We used a dissociation energy of $3.465 \mathrm{eV}$. The abundances of $\mathrm{C}$ and $\mathrm{O}$ are coupled through the $\mathrm{CO}$ molecule. In the absence of an $\mathrm{O}$ abundance measurement, we arbitrarily adopted a halo-like value of $[\mathrm{O} / \mathrm{Fe}]=+0.4$, and note that for our program stars, the derived $\mathrm{C}$ abundance is insensitive to the adopted $\mathrm{O}$ abundance (i.e., for a handful of stars, we adopted $[\mathrm{O} / \mathrm{Fe}]=0.0$ and $[\mathrm{O} / \mathrm{Fe}]=+1.5$, and the derived $\mathrm{C}$ abundance was unchanged). The NH line list was the same as in Johnson et al. (2007), in which the Kurucz- $g f$ values were reduced by a factor of two, and the dissociation energy was $3.450 \mathrm{eV}$. Given the low S/N at these wavelengths, we smoothed the observed spectra with a 5 pixel boxcar function to increase the $\mathrm{S} / \mathrm{N}$ (at the expense of spectral resolution). The $\mathrm{N}$ abundance was adjusted until the synthetic spectra matched the observed spectra. In Figures 2-4, we show examples of the spectrum synthesis.

As with the analysis of Fe lines, we repeated the element abundance analysis using the subset of lines believed to be unaffected by $\mathrm{CH}$ blends, combined with the stellar parameters obtained using the set of "CH clean" Fe lines. Depending on whether the star was found to be a CEMP object, we adopted the element abundances associated with the relevant element abundance analysis. In particular, we note that our line list has only one Si line, $3905.52 \AA$, and that line is excluded in the "CH clean" set of lines. Therefore, there are CEMP stars with Si equivalent-width measurements in Paper I without Si abundance measurements. For these CEMP stars, we tried to measure $\mathrm{Si}$ abundances (or limits) from spectrum synthesis of the $4102.94 \AA$ Si line. We present abundance ratios in Table 2 ( $\mathrm{C}$ and $\mathrm{N}$ ) and Table 3 (Na to $\mathrm{Ba}$ ). The adopted solar abundances for all elements were from Asplund et al. (2009).

\subsection{Abundance Uncertainties}

Our abundance measurements are subject to uncertainties in the model parameters. We estimated these uncertainties to 


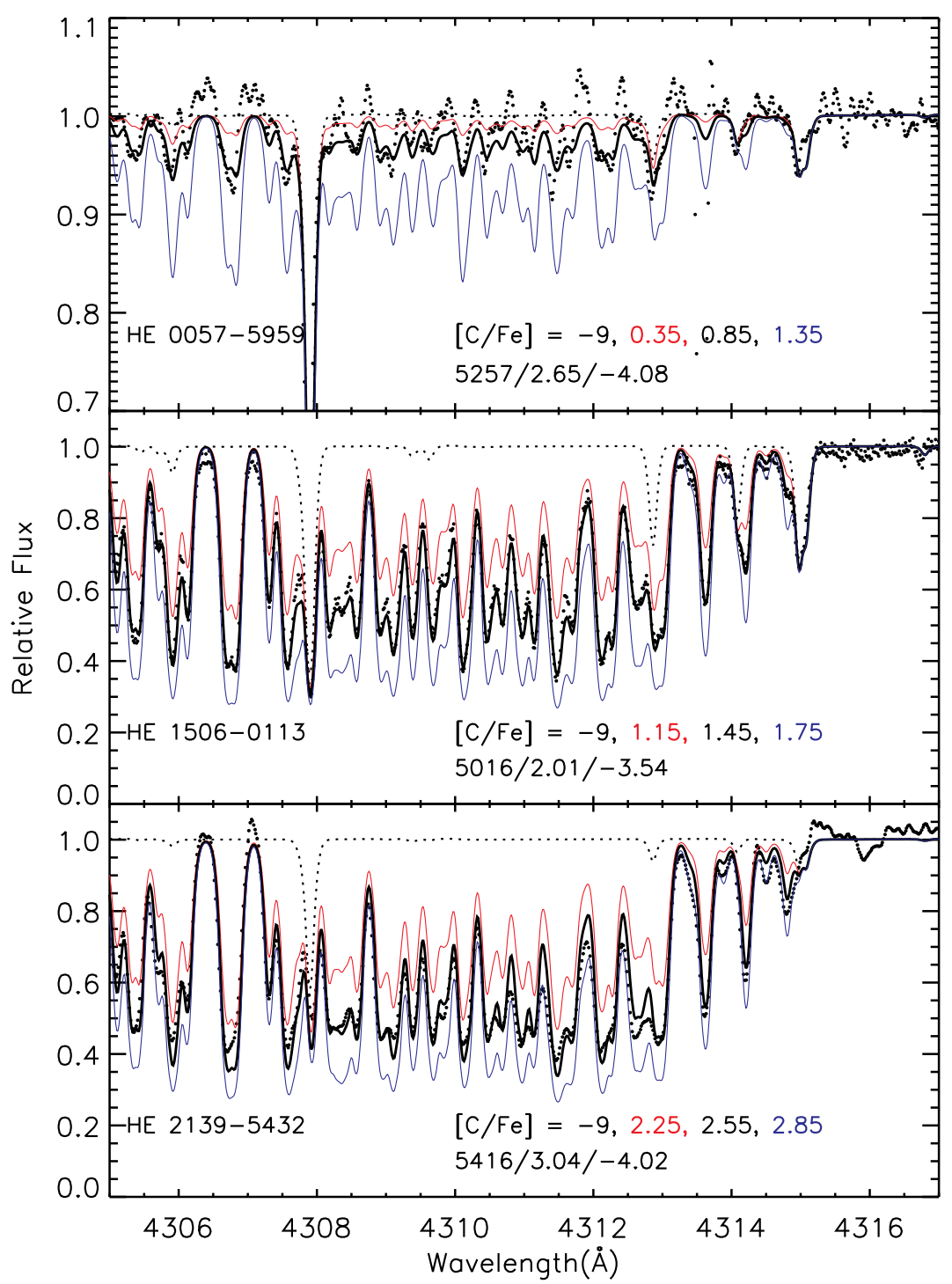

Figure 2. Comparison of observed (filled dots) and synthetic spectra in the region $4305-4317 \AA$ A Synthetic spectra with no C, [C/Fe] $=-9$, are shown as thin dotted lines. The best-fitting synthetic spectra are the thick black lines. Unsatisfactory fits are shown as thin red and blue lines. The stellar parameters $T_{\text {eff }} / \log g /[$ Fe $/ \mathrm{H}]$ are shown.

(A color version of this figure is available in the online journal.)

be $T_{\text {eff }} \pm 100 \mathrm{~K}, \log g \pm 0.3 \mathrm{dex}, \xi_{t} \pm 0.3 \mathrm{~km} \mathrm{~s}^{-1}$, and $[\mathrm{M} / \mathrm{H}] \pm 0.3$ dex. To determine the abundance errors, we repeated our analysis varying our parameters, one at a time, assuming that the errors are symmetric for positive and negative changes. We present these abundance uncertainty estimates in Table 4 , in which the final column is the accumulated error when the four uncertainties are added quadratically. Strictly speaking, quadratic addition is appropriate if the errors are fully independent. Additionally, our approach neglects covariances, and we refer the interested reader to McWilliam et al. (1995), Johnson (2002), and Barklem et al. (2005) for a more detailed discussion. Note that the contribution from the uncertainties in $[\mathrm{M} / \mathrm{H}]$ to the total error budget is small, in general. Therefore, our condition to re-compute a model atmosphere only when $\left|[\mathrm{M} / \mathrm{H}]_{\text {model }}-[\mathrm{Fe} / \mathrm{H}]_{\text {star }}\right|>0.1$ dex does not adversely affect our results.

To obtain the total error estimates given in Table 3, we follow Norris et al. (2010b). We replace the random error in Table 3 (s.e. $\log \epsilon$ ) by $\max \left(\right.$ s.e. $\log \epsilon, 0.20 / \sqrt{N_{\operatorname{lines}}}$ ) where the second term is what would be expected for a set of $N_{\text {lines }}$ with a dispersion of 0.20 dex (a conservative estimate for the abundance dispersion of Fe I lines). The total error is obtained by quadratically adding this updated random error with the systematic error in Table 4. Finally, we note that this 1D LTE analysis is subject to abundance uncertainties from three-dimensional (3D) and non-LTE effects (Asplund 2005).

\subsection{Comparison Using Different Model Atmospheres}

For a subset of the program stars, we computed abundances using the MARCS (Gustafsson et al. 2008) grid of model atmospheres. Three stars were chosen to sample a reasonable range of stellar parameters: two giants and one main-sequence turnoff star. In Table 5 we show the abundance differences for $A(\mathrm{X})$, in the sense Castelli \& Kurucz (2003) - MARCS. For these representative objects, the maximum abundance difference was $\Delta A(\mathrm{X})=0.05 \mathrm{dex}$, and the minimum abundance difference was $\Delta A(\mathrm{X})=-0.02$ dex. When considering the ratio $[\mathrm{X} / \mathrm{Fe}]$, we note that the maximum abundance difference was $\Delta[\mathrm{X} / \mathrm{Fe}]=$ 


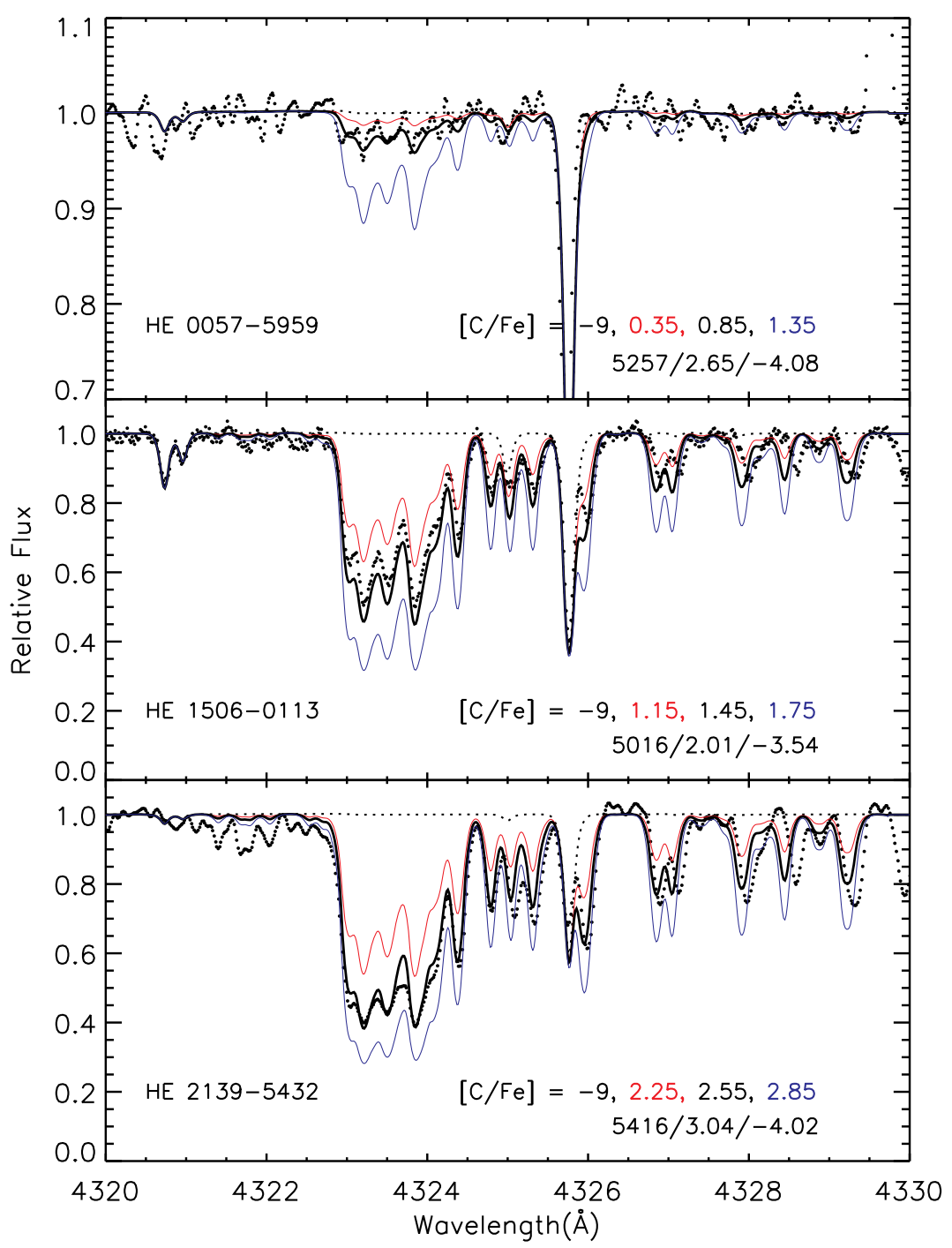

Figure 3. Same as Figure 2, but for the region 4320-4330 ̊.

(A color version of this figure is available in the online journal.)

$0.02 \mathrm{dex}$, and the minimum abundance difference was $\Delta[\mathrm{X} / \mathrm{Fe}]=-0.01$ dex. We regard these abundance differences, $\Delta A(\mathrm{X})$ and $\Delta[\mathrm{X} / \mathrm{Fe}]$, to be small, especially when compared to the abundance uncertainties and errors in Tables 3 and 4. Therefore, we do not expect the choice of model atmosphere grid, Castelli \& Kurucz (2003) or MARCS (Gustafsson et al. 2008), to significantly alter our abundance results or subsequent interpretation. However, we note that a more complete chemical abundance analysis would require, among other things, tailored models with appropriate CNO abundances.

\section{COMPARISON WITH THE FIRST STARS ABUNDANCE SCALE}

In the context of elemental-abundance determinations in metal-poor stars, the First ST ARS group (e.g., Cayrel et al. 2004; Spite et al. 2005; François et al. 2007; Bonifacio et al. 2009) obtained very high quality spectra and conducted a careful analysis, such that their derived abundances for all elements exhibit very small scatter about the mean trends with metallicity. As already mentioned, Cayrel et al. (2004, abstract) highlight the importance of correct treatment of continuum scattering to "allow proper interpretation of the blue regions of the spectra." Therefore, we regard the following as an important test of our analysis of metal-poor stars, namely, Using the Cayrel et al. (2004) line list and atmospheric parameters, does our combination of model atmospheres (Castelli \& Kurucz) and line-analysis software (MOOG) reproduce their abundances from the OSMARCS model atmospheres (Gustafsson et al. 1975) and the Turbospectrum (Alvarez \& Plez 1998) lineanalysis software?

In Figure 5, we compare our abundances with those of Cayrel et al. (2004), star by star, and find excellent agreement. (For $\mathrm{O}, \mathrm{Na}$, and $\mathrm{Mg}$, there are a handful of outliers, and on closer examination we find one outlier common to all three panels. This object is the most metal-rich and one of the warmest stars in the sample.) We note that our abundances were produced using the updated version of MOOG with appropriate continuum scattering routines. We conducted a similar test using the 2009 version of MOOG available on the Web. ${ }^{11}$ Although that version of MOOG, which does not include the newer continuum scattering routines, was also able to provide a good

\footnotetext{
$\overline{11 \text { http://www.as.utexas.edu/ chris/moog.html }}$
} 


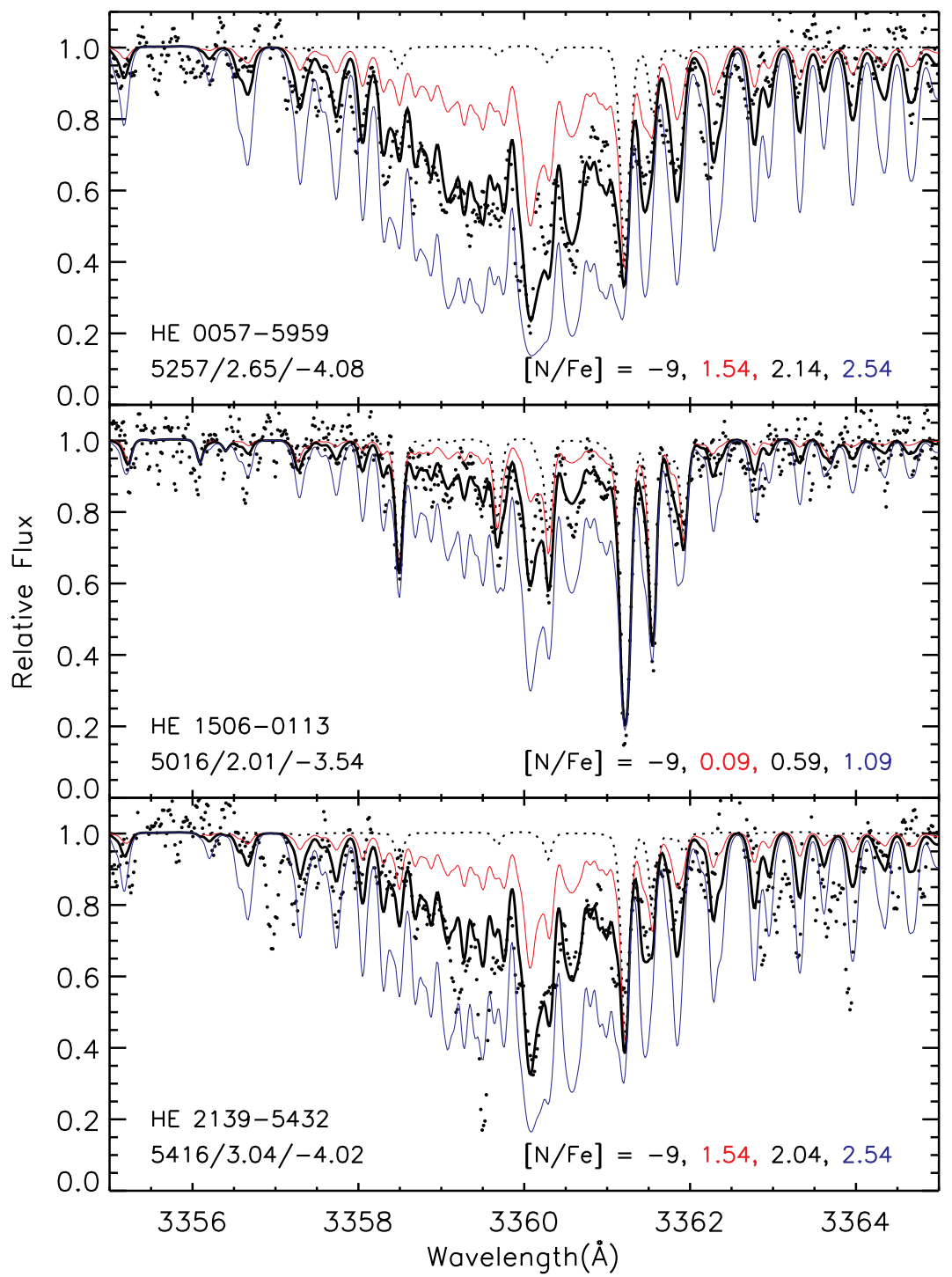

Figure 4. Same as Figure 2, but for [N/Fe] in the region 3355-3365 A.

(A color version of this figure is available in the online journal.)

match to the Cayrel et al. (2004) abundances, the version we employed produced superior agreement. As expected, the abundance differences between Cayrel et al. (2004) and the 2009 version of MOOG exhibited a strong wavelength dependence toward the blue. Therefore, the results of this test show that our "machinery" (Castelli \& Kurucz models atmospheres and the MOOG spectrum synthesis program) reproduces the Cayrel et al. (2004) abundances, when adopting the same atmospheric parameters.

For $\mathrm{C}$ and $\mathrm{N}$, we conducted the following test. In the course of our observing campaigns, described in Paper I, we obtained high-S/N spectra of the metal-poor standards BD $-18^{\circ} 5550$, $\mathrm{CD}-38^{\circ}$ 245, and CS 22892-052. Using the Cayrel et al. (2004) stellar parameters, we derived $[\mathrm{C} / \mathrm{H}]$ and $[\mathrm{N} / \mathrm{H}]$ from our spectra. (Our Keck spectrum of BD $-18^{\circ} 5550$ did not include the $3360 \AA \mathrm{NH}$ lines and for $\mathrm{CD}-38^{\circ} 245$, Spite et al. 2005 only give an upper limit for C.) For $[\mathrm{C} / \mathrm{H}]$, our abundances were within 0.02 dex and 0.08 dex of the Spite et al. (2005) values for BD $-18^{\circ} 5550$ and CS 22892-052, respectively. For $[\mathrm{N} / \mathrm{H}]$, our abundances were within $0.05 \mathrm{dex}$ and 0.10 dex of the Spite et al. (2005) values for CD $-38^{\circ} 245$ and CS 22892-052, respectively. We consider these abundance differences (this work - Spite et al.) to be small, and therefore regard the results of this test as a demonstration that our $\mathrm{C}$ and $\mathrm{N}$ abundances are on the Spite et al. (2005) scale, when using their stellar parameters.

Finally, we compared our $\mathrm{Sr}$ and $\mathrm{Ba}$ abundances to those of François et al. (2007), who measured relative abundances for some 16 neutron-capture elements using spectrum synthesis. For the metal-poor standards BD $-18^{\circ} 5550, \mathrm{CD}-38^{\circ}$ 245, and CS 22892-052, the abundance differences $\Delta[\mathrm{Sr} / \mathrm{H}]$ (This Study - François et al.) are $-0.08 \mathrm{dex},-0.09 \mathrm{dex}$, and -0.01 dex, respectively, while the abundance differences $\Delta[\mathrm{Ba} / \mathrm{H}]$ are $+0.06 \mathrm{dex},-0.02 \mathrm{dex}$, and $0.00 \mathrm{dex}$, respectively. Again, we consider these differences to be small, and hence this comparison demonstrates that our $\mathrm{Sr}$ and $\mathrm{Ba}$ abundances are on the François et al. (2007) scale, when using their stellar parameters. 
Table 2

$\mathrm{C}$ and $\mathrm{N}$ Abundances for the 38 Program Stars

\begin{tabular}{|c|c|c|c|c|}
\hline $\begin{array}{l}\text { Star } \\
(1)\end{array}$ & $\begin{array}{c}A(\mathrm{C}) \\
(2)\end{array}$ & $\begin{array}{c}{[\mathrm{C} / \mathrm{Fe}]} \\
(3)\end{array}$ & $\begin{array}{c}A(\mathrm{~N}) \\
(4)\end{array}$ & $\begin{array}{c}{[\mathrm{N} / \mathrm{Fe}]} \\
(5)\end{array}$ \\
\hline $52972-1213-507$ & 8.26 & 2.82 & $\cdots$ & $\cdots$ \\
\hline $53327-2044-515^{a}$ & 5.56 & 1.13 & $\cdots$ & $\cdots$ \\
\hline $53327-2044-515^{b}$ & 5.91 & 1.57 & $\cdots$ & $\cdots$ \\
\hline 53436-1996-093 & $<6.56$ & $<1.66$ & $\cdots$ & $\cdots$ \\
\hline $54142-2667-094$ & $<6.86$ & $<1.39$ & $\cdots$ & $\cdots$ \\
\hline BS 16545-089 & $<6.76$ & $<1.77$ & $\cdots$ & $\cdots$ \\
\hline CS 30336-049 & $<4.56$ & $<0.23$ & 4.7 & 0.97 \\
\hline HE 0049-3948 & $<6.56$ & $<1.81$ & $<6.55$ & $<2.40$ \\
\hline HE 0057-5959 & 5.21 & 0.86 & 5.9 & 2.15 \\
\hline HE $0102-1213$ & $<6.46$ & $<1.31$ & $\cdots$ & $\cdots$ \\
\hline HE $0146-1548$ & 5.81 & 0.84 & $\cdots$ & $\cdots$ \\
\hline HE $0207-1423$ & 7.86 & 2.38 & $\cdots$ & $\cdots$ \\
\hline HE $0228-4047^{a}$ & $<6.56$ & $<1.88$ & $\cdots$ & $\cdots$ \\
\hline HE $0228-4047^{b}$ & $<6.66$ & $<1.98$ & $\cdots$ & $\cdots$ \\
\hline HE $0231-6025$ & $<6.96$ & $<1.64$ & $\cdots$ & $\ldots$ \\
\hline HE $0253-1331$ & $<7.06$ & $<1.64$ & $\cdots$ & $\cdots$ \\
\hline HE 0314-1739 & $<7.06$ & $<1.49$ & $\cdots$ & $\cdots$ \\
\hline HE $0355-3728^{a}$ & $<7.16$ & $<2.14$ & $\cdots$ & $\cdots$ \\
\hline HE $0355-3728^{\mathrm{b}}$ & $<7.36$ & $<2.34$ & $\cdots$ & $\cdots$ \\
\hline HE $0945-1435^{\mathrm{a}}$ & $<6.36$ & $<1.70$ & $\cdots$ & $\cdots$ \\
\hline HE $0945-1435^{b}$ & $<6.46$ & $<1.81$ & $\cdots$ & $\cdots$ \\
\hline HE $1055+0104^{\mathrm{a}}$ & $<6.76$ & $<1.20$ & $\cdots$ & $\cdots$ \\
\hline HE $1055+0104^{b}$ & $<6.96$ & $<1.42$ & $\cdots$ & $\cdots$ \\
\hline HE $1116-0054^{\mathrm{a}}$ & $<6.66$ & $<1.72$ & $<7.25$ & $<2.91$ \\
\hline HE $1116-0054^{b}$ & $<6.86$ & $<1.92$ & $<7.25$ & $<2.91$ \\
\hline HE $1142-1422$ & $<7.16$ & $<1.57$ & $<7.50$ & $<2.51$ \\
\hline HE $1201-1512^{d}$ & 5.71 & 1.14 & $<5.20$ & $<1.23$ \\
\hline HE $1201-1512^{d}$ & 6.11 & 1.6 & $<5.20$ & $<1.29$ \\
\hline HE 1204-0744 & $<7.26$ & $<1.55$ & $\cdots$ & $\cdots$ \\
\hline HE $1207-3108$ & $<5.46$ & $<-0.27$ & 5.55 & 0.42 \\
\hline HE 1320-2952 & $<5.26$ & $<0.52$ & $<5.00$ & $<0.86$ \\
\hline HE $1346-0427^{a}$ & $<5.96$ & $<1.10$ & $\cdots$ & $\cdots$ \\
\hline HE $1346-0427^{b}$ & $<6.16$ & $<1.31$ & $\cdots$ & $\cdots$ \\
\hline HE $1402-0523^{a}$ & $<6.76$ & $<1.50$ & $<6.50$ & $<1.84$ \\
\hline HE $1402-0523^{b}$ & $<6.86$ & $<1.62$ & $<6.60$ & $<1.96$ \\
\hline HE 1506-0113 & 6.36 & 1.47 & 4.9 & 0.61 \\
\hline HE 2020-5228 & $<7.16$ & $<1.66$ & $<7.20$ & $<2.30$ \\
\hline HE 2032-5633 & $<7.16$ & $<2.36$ & $<6.80$ & $<2.60$ \\
\hline HE 2047-5612 & $<6.66$ & $<1.37$ & $<6.60$ & $<1.91$ \\
\hline HE 2135-1924 & $<6.86$ & $<1.74$ & $\cdots$ & $\ldots$ \\
\hline HE 2136-6030 & $<7.26$ & $<1.71$ & $\cdots$ & $\cdots$ \\
\hline HE 2139-5432 & 7.01 & 2.59 & 5.9 & 2.08 \\
\hline HE 2141-0726 & $<7.26$ & $<1.55$ & $\cdots$ & $\cdots$ \\
\hline HE 2142-5656 & 6.51 & 0.95 & 5.5 & 0.54 \\
\hline HE $2202-4831$ & 8.06 & 2.41 & $\cdots$ & $\cdots$ \\
\hline HE $2246-2410$ & $<6.86$ & $<1.39$ & $<7.15$ & $<2.28$ \\
\hline HE $2247-7400$ & 6.26 & 0.7 & $\ldots$ & $\cdots$ \\
\hline
\end{tabular}

Notes.

${ }^{\text {a }}$ For this set of results, a dwarf gravity is assumed (see Section 2.1 for details).

${ }^{b}$ For this set of results, a subgiant gravity is assumed (see Section 2.1 for details).

\section{RE-ANALYSIS OF THE LITERATURE SAMPLE}

Having completed the analysis of the program stars, we then sought to undertake a homogeneous re-analysis of all extremely metal-poor Galactic stars with $[\mathrm{Fe} / \mathrm{H}] \leqslant-3.0$. We queried the SAGA database (Suda et al. 2008) for all stars with $[\mathrm{Fe} / \mathrm{H}] \leqslant-2.9$, with the aim of re-analyzing those stars using the published equivalent widths, but with our analysis procedures and techniques. The rationale for choosing $[\mathrm{Fe} / \mathrm{H}]=$ -2.9 as the cutoff was that we were hoping to find as many stars
Table 3

Chemical Abundances (Na-Ba) for the Program Stars

\begin{tabular}{lccccc}
\hline \hline $\begin{array}{l}\text { Star } \\
(1)\end{array}$ & $\begin{array}{c}A(\mathrm{X}) \\
(2)\end{array}$ & $\begin{array}{c}N_{\text {lines }} \\
(3)\end{array}$ & $\begin{array}{c}\text { s.e. } \log { }^{\mathrm{a}} \\
(4)\end{array}$ & $\begin{array}{c}\text { Total Error }^{\mathrm{b}} \\
(5)\end{array}$ & $\begin{array}{c}{[\mathrm{X} / \mathrm{Fe}]} \\
(6)\end{array}$ \\
\hline \multicolumn{7}{c}{$\mathrm{Na}$} \\
\hline $52972-1213-507$ & 4.86 & 2 & 0.32 & 0.33 & 1.60 \\
$53327-2044-515^{\mathrm{c}}$ & 2.35 & 1 & $\ldots$ & $\ldots$ & 0.11 \\
$53327-2044-515^{\mathrm{d}}$ & 2.32 & 1 & $\ldots$ & $\ldots$ & 0.17 \\
$53436-1996-093$ & 2.58 & 1 & $\ldots$ & $\ldots$ & -0.13 \\
$54142-2667-094$ & $\ldots$ & $\ldots$ & $\ldots$ & $\ldots$ & $\ldots$ \\
\hline
\end{tabular}

Notes.

a Standard error of the mean.

${ }^{b}$ Total error is the quadratic sum of the updated random error and the systematic error (see Section 2.3 for details).

${ }^{\mathrm{c}}$ Dwarf gravity is assumed (see Section 2.1 for details).

${ }^{\mathrm{d}}$ Subgiant gravity is assumed (see Section 2.1 for details).

e Abundances, or limits, were determined from spectrum synthesis of the $4102.94 \AA$ A Si line.

f Abundances limits were determined from the $4077.71 \AA$ Sr line.

g Abundances limits were determined from the $4554.03 \AA$ Ba line

(This table is available in its entirety in a machine-readable form in the online journal. A portion is shown here for guidance regarding its form and content.)

Table 4

Abundance Errors from Uncertainties in Atmospheric Parameters for the 38 Program Stars

\begin{tabular}{lccccc}
\hline \hline Species & $\begin{array}{c}\Delta T_{\text {eff }} \\
(100 \mathrm{~K}) \\
(2)\end{array}$ & $\begin{array}{c}\Delta \log g \\
(0.3 \mathrm{dex}) \\
(1)\end{array}$ & $\begin{array}{c}\Delta \xi_{t} \\
\left(0.3 \mathrm{~km} \mathrm{~s}^{-1}\right)\end{array}$ & $\begin{array}{c}\Delta[\mathrm{M} / \mathrm{H}] \\
(0.3 \mathrm{dex}) \\
(5)\end{array}$ & $\begin{array}{c}\Delta[\mathrm{X} / \mathrm{Fe}] \\
(\mathrm{dex}) \\
(6)\end{array}$ \\
\hline \multicolumn{7}{c}{$52972-1213-507$} & & \\
\hline$\Delta[\mathrm{Na} / \mathrm{Fe}]$ & 0.00 & -0.08 & 0.00 & 0.04 & 0.09 \\
$\Delta[\mathrm{Mg} / \mathrm{Fe}]$ & -0.01 & -0.05 & 0.00 & 0.04 & 0.06 \\
$\Delta[\mathrm{Al} / \mathrm{Fe}]$ & $\ldots$ & $\ldots$ & $\ldots$ & $\ldots$ & $\ldots$ \\
$\Delta[\mathrm{Si} / \mathrm{Fe}]$ & $\ldots$ & $\ldots$ & $\ldots$ & $\ldots$ & $\ldots$ \\
$\Delta[\mathrm{Ca} / \mathrm{Fe}]$ & 0.01 & -0.07 & 0.00 & -0.03 & 0.08
\end{tabular}

Notes.

${ }^{\text {a }}$ Dwarf gravity is assumed (see Section 2.1 for details).

${ }^{\mathrm{b}}$ Subgiant gravity is assumed (see Section 2.1 for details).

(This table is available in its entirety in a machine-readable form in the online journal. A portion is shown here for guidance regarding its form and content.)

as possible with $[\mathrm{Fe} / \mathrm{H}] \leqslant-3.0$ on our scale, some of which may have higher published metallicities. At the same time, we needed to ensure a manageable sample.

At the time of our SAGA query (2010 February 2), the database had been last updated on 2009 September 2 . Our query returned 196 stars with $[\mathrm{Fe} / \mathrm{H}] \leqslant-2.9$; from this list of stars we identified 16 references in each of which reliable equivalent widths had been published for several stars, based on highquality spectra. These 16 references included a large number of stars in the SAGA database with $[\mathrm{Fe} / \mathrm{H}] \leqslant-3.0$ (notable exceptions include stars unique to Barklem et al. 2005, BD +44 493 with $[\mathrm{Fe} / \mathrm{H}]=-3.7$ [Ito et al. 2009], the C-rich dwarf G77-61 with $[\mathrm{Fe} / \mathrm{H}]=-4.03$ [Plez \& Cohen 2005], SDSS $\mathrm{J} 102915+172927$ with $[\mathrm{Fe} / \mathrm{H}]_{1 \mathrm{DLTE}}=-4.73[\mathrm{Caffau}$ et al. 2011], and recent papers by Hollek et al. 2011 and Sbordone et al. 2012). It is also worth noting that this is a study of Galactic stars, and so we did not consider any of the growing number of stars in dwarf galaxies with $[\mathrm{Fe} / \mathrm{H}] \leqslant-3$, even though we have analyzed several such objects using very similar techniques to those in this series of papers (e.g., Norris et al. 2010a, 2010b). 


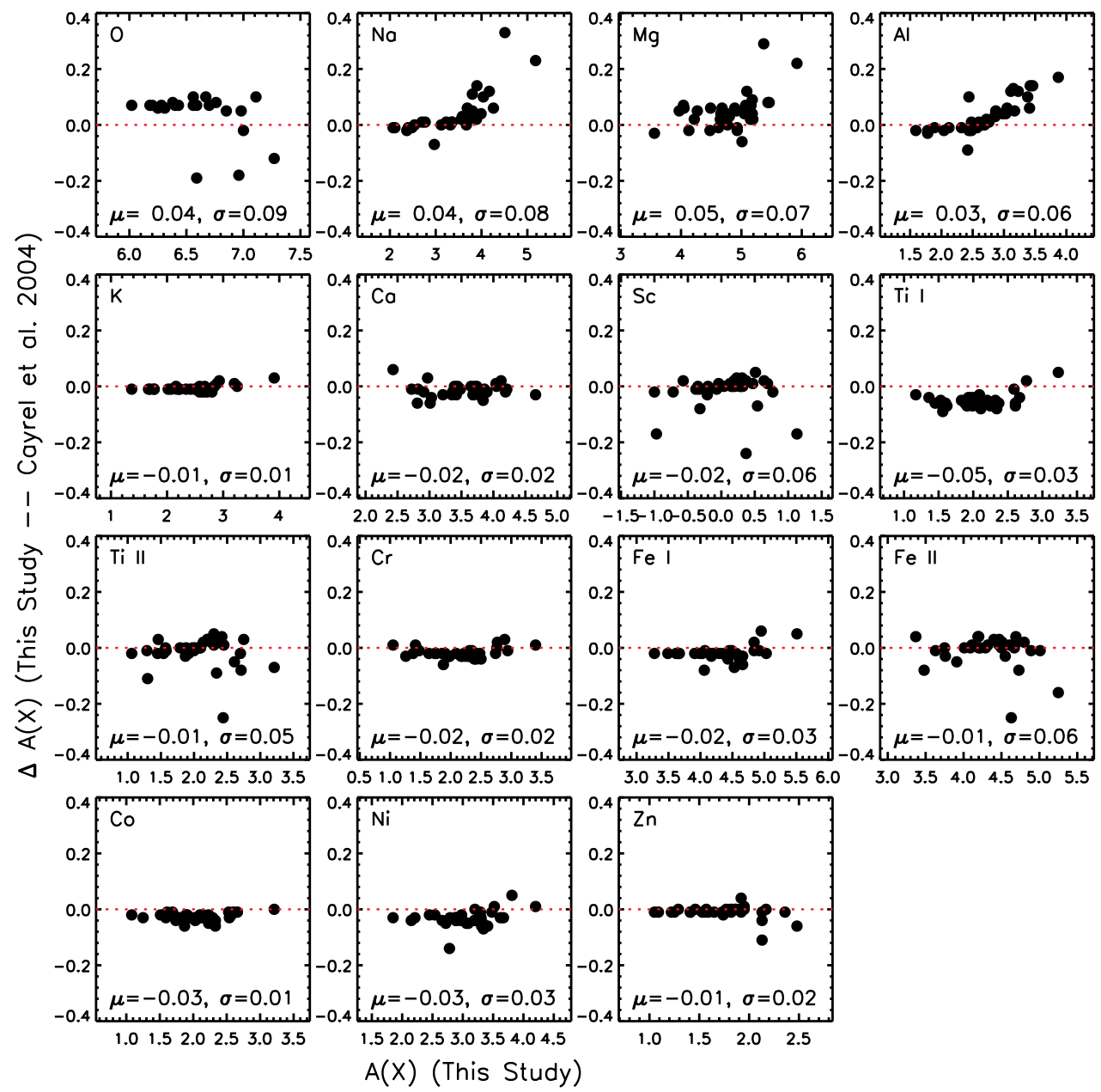

Figure 5. Star-by-star comparison between the Cayrel et al. (2004) abundances and our abundances when using their line list and atmospheric parameters. The numbers at the bottom of each panel are the mean (Our Analysis - Cayrel) and the dispersion.

(A color version of this figure is available in the online journal.)

Table 5

Abundance Differences between the Castelli \& Kurucz (2003) and MARCS (Gustafsson et al. 2008) Model Atmospheres for Three Representative Stars

\begin{tabular}{|c|c|c|c|}
\hline $\begin{array}{l}\text { Species } \\
\text { (1) }\end{array}$ & $\begin{array}{c}\text { HE } 0057-5959^{a} \\
\text { (2) }\end{array}$ & $\begin{array}{c}\text { HE } 1320-2952^{b} \\
\text { (3) }\end{array}$ & $\begin{array}{c}\text { HE } 2032-5633^{\mathrm{c}} \\
\text { (4) }\end{array}$ \\
\hline$\Delta A(\mathrm{Na})$ & 0.03 & 0.03 & -0.01 \\
\hline$\Delta A(\mathrm{Mg})$ & 0.01 & 0.03 & -0.01 \\
\hline$\Delta A(\mathrm{Al})$ & $\ldots$ & 0.02 & -0.01 \\
\hline$\Delta A(\mathrm{Si})$ & $\ldots$ & 0.03 & 0.00 \\
\hline$\Delta A(\mathrm{Ca})$ & 0.01 & 0.02 & -0.02 \\
\hline$\Delta A(\mathrm{Sc})$ & 0.03 & 0.03 & $\cdots$ \\
\hline$\Delta A($ Ti I $)$ & 0.02 & 0.02 & $\ldots$ \\
\hline$\Delta A(\mathrm{Ti}$ II $)$ & 0.02 & 0.04 & 0.01 \\
\hline$\Delta A(\mathrm{Cr})$ & 0.01 & 0.02 & $\ldots$ \\
\hline$\Delta A(\mathrm{Mn})$ & $\cdots$ & 0.02 & $\cdots$ \\
\hline$\Delta A\left(\mathrm{Fe}_{\mathrm{I}}\right)$ & 0.02 & 0.03 & -0.01 \\
\hline$\Delta A\left(\mathrm{Fe}_{\mathrm{II}}\right)$ & $\cdots$ & $\cdots$ & $\cdots$ \\
\hline$\Delta A(\mathrm{Co})$ & $\ldots$ & 0.01 & $\ldots$ \\
\hline$\Delta A(\mathrm{Ni})$ & 0.02 & 0.02 & $\ldots$ \\
\hline$\Delta A(\mathrm{Sr})$ & 0.03 & 0.05 & $\ldots$ \\
\hline$\Delta A(\mathrm{Ba})$ & 0.03 & 0.04 & $\cdots$ \\
\hline
\end{tabular}

Notes.

${ }^{\mathrm{a}} \mathrm{HE}$ 0057-5959: $T_{\text {eff }}=5257 \mathrm{~K}, \log g=2.65,[\mathrm{Fe} / \mathrm{H}]=-4.08$.

${ }^{\mathrm{b}} \mathrm{HE} 1320-2952: T_{\text {eff }}=5106 \mathrm{~K}, \log g=2.26,[\mathrm{Fe} / \mathrm{H}]=-3.69$.

${ }^{\mathrm{c}} \mathrm{HE} 2032-5633: T_{\mathrm{eff}}=6457 \mathrm{~K}, \log g=3.78,[\mathrm{Fe} / \mathrm{H}]=-3.63$.
The 16 references we selected contained some 207 stars, many more metal-rich than $[\mathrm{Fe} / \mathrm{H}]=-3.0$. Nevertheless, we analyzed all 207 stars in the following manner.

We defined "giants" as those stars with $\log g<3$, and "dwarfs" as those stars with $\log g>3$, where the surface gravities were taken from the literature sources. ${ }^{12}$ With the exception of the three most metal-poor stars, which we shall discuss below, we then used the published photometry, reddenings, and metallicities, $[\mathrm{Fe} / \mathrm{H}]$, together with the infrared flux method (IRFM) metallicity-dependent color-temperature relations adopting Casagrande et al. (2010) for the dwarfs and Ramírez \& Meléndez (2005) for the giants, to determine effective temperatures. We note that the Ramírez \& Meléndez (2005) calibration is valid only for $[\mathrm{Fe} / \mathrm{H}]>-4.0$. For a small number of stars, our $T_{\text {eff }}$ values involve a small extrapolation down to $[\mathrm{Fe} / \mathrm{H}]=-4.2$. For a subset of these literature stars, observations and analysis using the spectrophotometric procedures described in Paper I yielded $T_{\text {eff. }}$. We refer to these $T_{\text {eff }}$ values as the "Bessell temperatures." For these stars with both "Bessell temperatures" and IRFM temperatures, we found average $T_{\text {eff }}$

\footnotetext{
12 Clearly our definition of "dwarfs" will include many subgiants and stars near the base on the giant branch. Nevertheless, we needed to define a boundary to separate dwarfs from giants and to then apply the different color-temperature relations to determine effective temperatures. Throughout the rest of the paper, these definitions for dwarfs and giants apply.
} 
Table 6

Model Atmosphere Parameters and $[\mathrm{Fe} / \mathrm{H}]$ for the Literature Sample

\begin{tabular}{|c|c|c|c|c|c|c|c|c|c|}
\hline Star & $\begin{array}{c}\text { RA2 } 2000^{\mathrm{a}} \\
\text { (2) }\end{array}$ & $\begin{array}{c}\text { DEC } 2000^{\mathrm{a}} \\
\text { (3) }\end{array}$ & $\begin{array}{l}T_{\text {eff }} \\
(\mathrm{K}) \\
(4)\end{array}$ & $\begin{array}{c}\log g \\
(\mathrm{cgs}) \\
(5)\end{array}$ & $\begin{array}{c}\xi_{t} \\
\left(\mathrm{~km} \mathrm{~s}^{-1}\right) \\
(6)\end{array}$ & $\begin{array}{c}{[\mathrm{M} / \mathrm{H}]_{\text {model }}} \\
\text { (7) }\end{array}$ & {$[\mathrm{Fe} / \mathrm{H}]_{\text {derived }}$} & $\begin{array}{c}\text { C-rich } \\
\text { b } \\
\text { (9) }\end{array}$ & Source \\
\hline CS 22957-022 & 000145.5 & -054946.6 & 5146 & 2.40 & 1.5 & -2.9 & -2.92 & 0 & 14 \\
\hline CS 29503-010 & 000455.4 & -242419.3 & 6570 & 4.25 & 1.3 & -1.0 & -1.00 & 1 & 3 \\
\hline CS $31085-024$ & 000827.9 & +105419.8 & 5778 & 4.64 & 0.3 & -2.8 & -2.80 & 0 & 14 \\
\hline BS $17570-063$ & 002036.2 & +234737.7 & 6233 & 4.46 & 0.8 & -3.0 & -2.95 & 0 & 5 \\
\hline HE 0024-2523 & 002727.7 & -250628.2 & 6635 & 4.11 & 1.2 & -2.8 & -2.82 & 0 & 6 \\
\hline
\end{tabular}

Notes.

${ }^{a}$ Coordinates are from the 2MASS database (Skrutskie et al. 2006).

b $1=$ CEMP object, adopting the Aoki et al. (2007) definition and $0=$ C-normal (see Section 7.1 for details).

Reference. (1) Aoki et al. 2002; (2) Aoki et al. 2006; (3) Aoki et al. 2007; (4) Aoki et al. 2008; (5) Bonifacio et al. 2007, 2009; (6) Carretta et al. 2002; Cohen et al. 2002; (7) Cayrel et al. 2004; (8) Christlieb et al. 2004; (9) Cohen et al. 2004; (10) Cohen et al. 2006; (11) Cohen et al. 2008; (12) Frebel et al. 2007; (13) Honda et al. 2004; (14) Lai et al. 2008; (15) Norris et al. 2001; (16) Norris et al. 2007.

(This table is available in its entirety in a machine-readable form in the online journal. A portion is shown here for guidance regarding its form and content.)

offsets of $+19 \mathrm{~K} \pm 42 \mathrm{~K}$ (Bessell - Casagrande et al. 2010) and $-45 \mathrm{~K} \pm 19 \mathrm{~K}$ (Bessell - Ramírez \& Meléndez 2005) from the IRFM for dwarfs and giants, respectively. (We note that obtaining these offsets involved an iterative process since the derived $T_{\text {eff }}$ values are weakly dependent on the adopted metallicity and the adopted metallicity changed as we employed the updated $T_{\text {eff }}$.) Finally, we took into account the $51 \mathrm{~K}$ average difference between the "Bessell temperatures" and our final temperatures, as determined from our 38 program stars (see Paper I). In summary, we applied corrections of $\Delta T_{\text {eff }}=+19 \mathrm{~K}+51 \mathrm{~K}=+70 \mathrm{~K}$ to the Casagrande et al. (2010) IRFM $T_{\text {eff }}$ for dwarf stars, and $\Delta T_{\text {eff }}=-45 \mathrm{~K}+51 \mathrm{~K}=+6 \mathrm{~K}$ to the Ramírez \& Meléndez (2005) IRFM $T_{\text {eff }}$ for giant stars.

Using our line list, presented in Paper I, we adopted the literature equivalent widths for lines in common with our list, and ignored lines that were not in common. This ensured that the $\log g f$ values were homogeneous. As demonstrated in Paper I, our equivalent-width measurements are on the same scale as various literature studies. For the surface gravity, we followed our analysis procedure for the program stars in which $\log g$ was determined from the $Y^{2}$ isochrones (Demarque et al. 2004), assuming the revised $T_{\text {eff }}$, an age of $10 \mathrm{Gyr}$, and $[\alpha / \mathrm{Fe}]=+0.3$. The published surface gravities were used for dwarf/subgiant discrimination. The microturbulent velocity was determined in the usual way, by forcing the abundances from Fe I lines to show no trend with reduced equivalent width. During this process, we removed Fe I lines having abundances that differed (1) from the median value by more than 0.5 dex or (2) from the median abundance by more than $3 \sigma$, as for our program stars, and the rejection applied to lines yielding abundances higher or lower than the median value. The average number of lines rejected per star was 4 , and the average number of lines per star was 49 . The largest number of rejected lines in a given star was 30 (of a total of 84 lines), and this star also has the highest fraction of rejected lines, $36 \%$. The result of this first-pass analysis was a revised estimate of the metallicity, $[\mathrm{Fe} / \mathrm{H}]$.

We then repeated the analysis using the updated metallicity. That is, we determined updated $T_{\text {eff }}$ using the IRFM calibrations, which are (weakly) sensitive to the assumed metallicity. In this second iteration, we again used the $Y^{2}$ isochrones and the published surface gravity for dwarf/subgiant classification, noting once more that this involves extrapolation beyond $[\mathrm{Fe} / \mathrm{H}]=$ -3.5 (down to $[\mathrm{Fe} / \mathrm{H}]=-4.2$ for the most metal-poor object in the literature sample). With these revised $T_{\text {eff }}$ and updated surface gravities, we computed new model atmospheres with the appropriate stellar parameters $\left(T_{\text {eff }}, \log g\right.$, and $[\mathrm{M} / \mathrm{H}]=$ $[\mathrm{Fe} / \mathrm{H}])$. For $T_{\text {eff }}$ and $\log g$, the revised values were generally very close to the initial values. The microturbulent velocity was determined and $\mathrm{Fe}$ I outliers removed using the criteria outlined above. The results of this second-pass analysis were final stellar parameters and metallicities for the literature sample, which are presented in Table 6. (For a small number of stars, a third iteration was necessary to ensure that the derived metallicity was sufficiently close to the value used to generate the model atmosphere, within 0.3 dex.) The evolutionary status, $T_{\text {eff }}$ versus $\log g$, for the literature sample is shown in the lower panel of Figure 1. We note that for our 38 program stars, the smallest number of Fe I lines measured in a given star was 14 . Therefore, for the literature sample, we discarded stars in which there were fewer than $14 \mathrm{Fe}$ I lines.

Following the procedures outlined above, we then determined element abundances using the published equivalent widths (only lines in common with our line list), final model atmospheres, and MOOG. Lines affected by hyperfine and/or isotopic splitting were treated appropriately. Chemical abundances for the literature sample are presented in Table 7 , where $[\mathrm{C} / \mathrm{Fe}]$ and $[\mathrm{N} / \mathrm{Fe}]$ are the values taken from the literature, but $[\mathrm{X} / \mathrm{Fe}]$ for $\mathrm{X}=\mathrm{Na}$ to $\mathrm{Ba}$ are recomputed on our homogeneous scale. As described in Yong et al. (2013; Paper III), we chose not to update the $[\mathrm{C} / \mathrm{Fe}]$ and $[\mathrm{N} / \mathrm{Fe}]$ abundances using our revised metallicities via $[\mathrm{C} / \mathrm{Fe}]_{\mathrm{New}}=[\mathrm{C} / \mathrm{Fe}]_{\text {Literature }}-\left([\mathrm{Fe} / \mathrm{H}]_{\text {This study }}-\right.$ $\left.[\mathrm{Fe} / \mathrm{H}]_{\text {Literature }}\right)$, since this approach only incorporates changes to the metallicity and does not include any changes to the $\mathrm{C}$ and/or $\mathrm{N}$ abundances. Furthermore, such an update does not affect our results or interpretation.

Following the procedure we adopted for the analysis of the program stars, we repeated the entire analysis of the literature sample using only a subset of lines believed to be free from $\mathrm{CH}$ blends. Depending on the published $[\mathrm{C} / \mathrm{Fe}]$ abundance ratio and the subsequent CEMP classification, we adopted the final stellar parameters and chemical abundances as determined using the appropriate line list and analysis.

The final sample of literature stars was reduced from 207 to 152 stars by the averaging of the results of stars having multiple analyses into a single set of abundances and removal of stars with fewer than $14 \mathrm{Fe}$ I lines. In all cases, there was excellent 


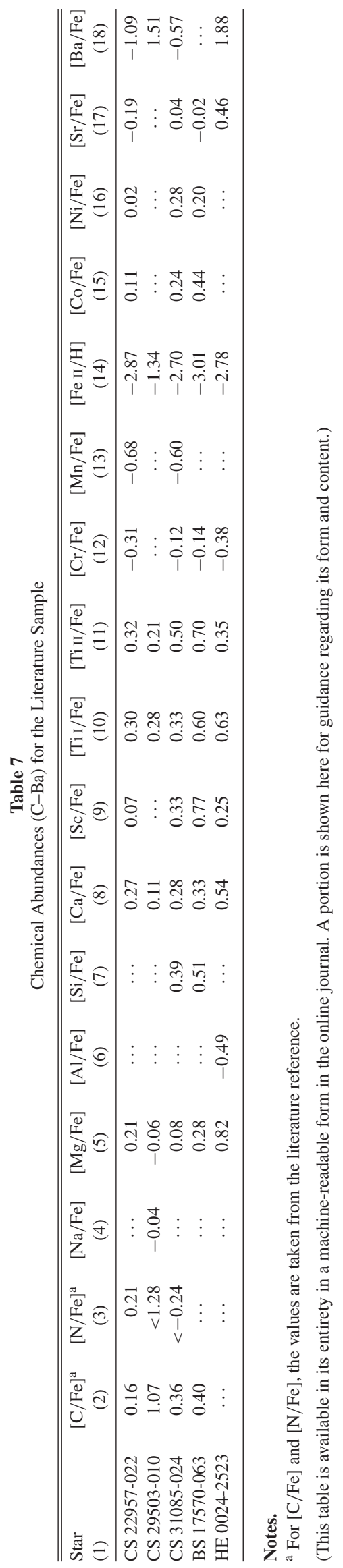


agreement for a given abundance ratio $[\mathrm{X} / \mathrm{Fe}]$ for stars having multiple analyses.

For the three HES stars with $[\mathrm{Fe} / \mathrm{H}]<-4.5$, HE 0107-5240 (Christlieb et al. 2002, 2004), HE 1327-2326 (Frebel et al. 2005; Aoki et al. 2006), and HE 0557-4840 (Norris et al. 2007), we did not attempt to re-derive effective temperatures or surface gravities. These stars lie in a metallicity regime in which the IRFM color-temperature relations are not calibrated. Therefore, we adopted the published stellar parameters $\left(T_{\text {eff }}, \log g, \xi_{t}\right.$, and $[\mathrm{M} / \mathrm{H}]$ ) and computed metallicities and chemical abundances using lines in common with our line list. Furthermore, we retain HE 1327-2326, despite the fact that only four Fe I lines (from a total of seven) were in common with our line list.

In summary, we have computed metallicities and chemical abundances for some 16 elements in 190 metal-poor Galactic stars (38 program stars and 152 literature stars). This is a homogeneous analysis with stellar parameters $\left(T_{\text {eff }}, \log g, \xi_{t}\right)$, metallicities, atomic data, solar abundances, and therefore abundance ratios, $[\mathrm{X} / \mathrm{Fe}]$, all on the same scale. For convenience, Table 9 includes coordinates, stellar parameters, and abundance ratios for all of the program stars and literature stars presented here.

\section{COMPARISON WITH PREVIOUS STUDIES}

In Figure 6, we compare our stellar parameters $\left(T_{\text {eff }}, \log g\right.$, and $[\mathrm{Fe} / \mathrm{H}]$ ) with the literature values. (In this comparison we exclude the three HES stars with $[\mathrm{Fe} / \mathrm{H}]<-4.5$, since we adopted their published $T_{\text {eff }}$ and $\log g$.) For all parameters, our revised values are, on average, in good agreement with the literature values. This is perhaps not surprising, given that many of the literature studies adopt similar approaches to determine these parameters. When comparing our values with the literature, the dispersion is comparable to our estimates of our internal uncertainties in stellar parameters.

Schörck et al. (2009) and Li et al. (2010) published metallicities for HES stars based on medium-resolution spectra. For our combined sample (program stars and literature stars), there are 12 stars in common with Schörck et al. (2009) and Li et al. (2010). Recall that there are program stars for which we conducted analyses assuming a dwarf gravity and a subgiant gravity. For the purposes of this comparison, we regard each analysis as an independent measurement. Thus, for these 12 stars we have $18[\mathrm{Fe} / \mathrm{H}]$ measurements. As noted in Paper III, our metallicities are lower than theirs by $0.26 \pm 0.06 \operatorname{dex}(\sigma=0.27)$.

We then compared the abundance ratios $[\mathrm{X} / \mathrm{Fe}]$, for $\mathrm{X}=\mathrm{Na}$ to $\mathrm{Ba}$, between the values computed in this study and the literature values. Our assumption was that our revised $[\mathrm{X} / \mathrm{Fe}]$ abundance ratios would be reasonably close to the literature values. Any differences in abundance ratios would presumably be driven by differences in the stellar parameters, model atmospheres, line-analysis software, atomic data, and/or solar abundances. As discussed, the stellar parameters are in good agreement. Similarly, we have shown that different model atmospheres and line-analysis software introduce only very small abundance differences. Finally, we do not anticipate large differences to arise from the solar abundances or atomic data. Therefore, for each element in each star, we plotted the abundance differences (this study - the literature). We fitted these differences for a given element with a Gaussian, and measured the FWHM, which ranged from 0.05 dex to 0.14 dex, with a mean of 0.10 dex. We then eliminated those stars in which the abundance differences exceeded $\max (0.50 \mathrm{dex}, 3 \sigma)$ from the average difference for a particular element (the average difference for a given element ranged from $-0.10 \mathrm{dex}$ to $+0.17 \mathrm{dex}$ ). That is, based on the
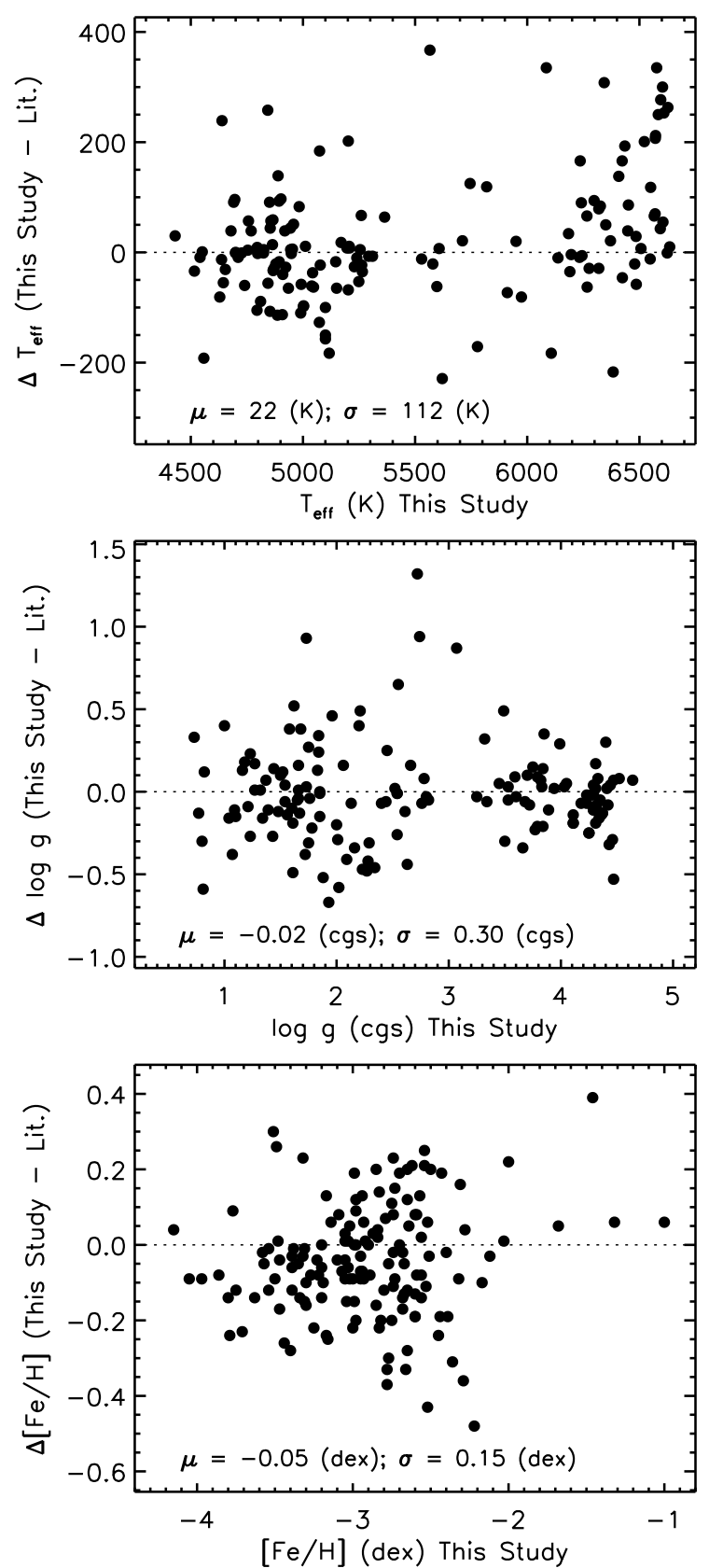

Figure 6. Star-by-star comparison of stellar parameters, $T_{\text {eff }}$ (upper panel), $\log g$ (middle panel), and $[\mathrm{Fe} / \mathrm{H}]$ (lower panel), for our re-analysis of the literature sample and the original literature values. The numbers at the bottom of each panel are the mean (our analysis - the literature) and the dispersion.

abundance differences, we removed particular elements from a given star. For example, a star may have an anomalous $[\mathrm{Mg} / \mathrm{Fe}]$ value, which is then removed. If all other elements in that star have $[\mathrm{X} / \mathrm{Fe}]$ ratios sufficiently close to the literature values, then those abundance ratios would be retained. For a given element, this resulted in fewer than seven stars being rejected, and we speculate that many of these outliers may be due to errors in the tables of equivalent widths, of which there are some 18,000 values. The abundance outliers are not included in Table 7 or in any other table or figure. Note that the three HES stars with $[\mathrm{Fe} / \mathrm{H}]<-4.5$ were included in this analysis, and there were no abundance outliers among these objects. 

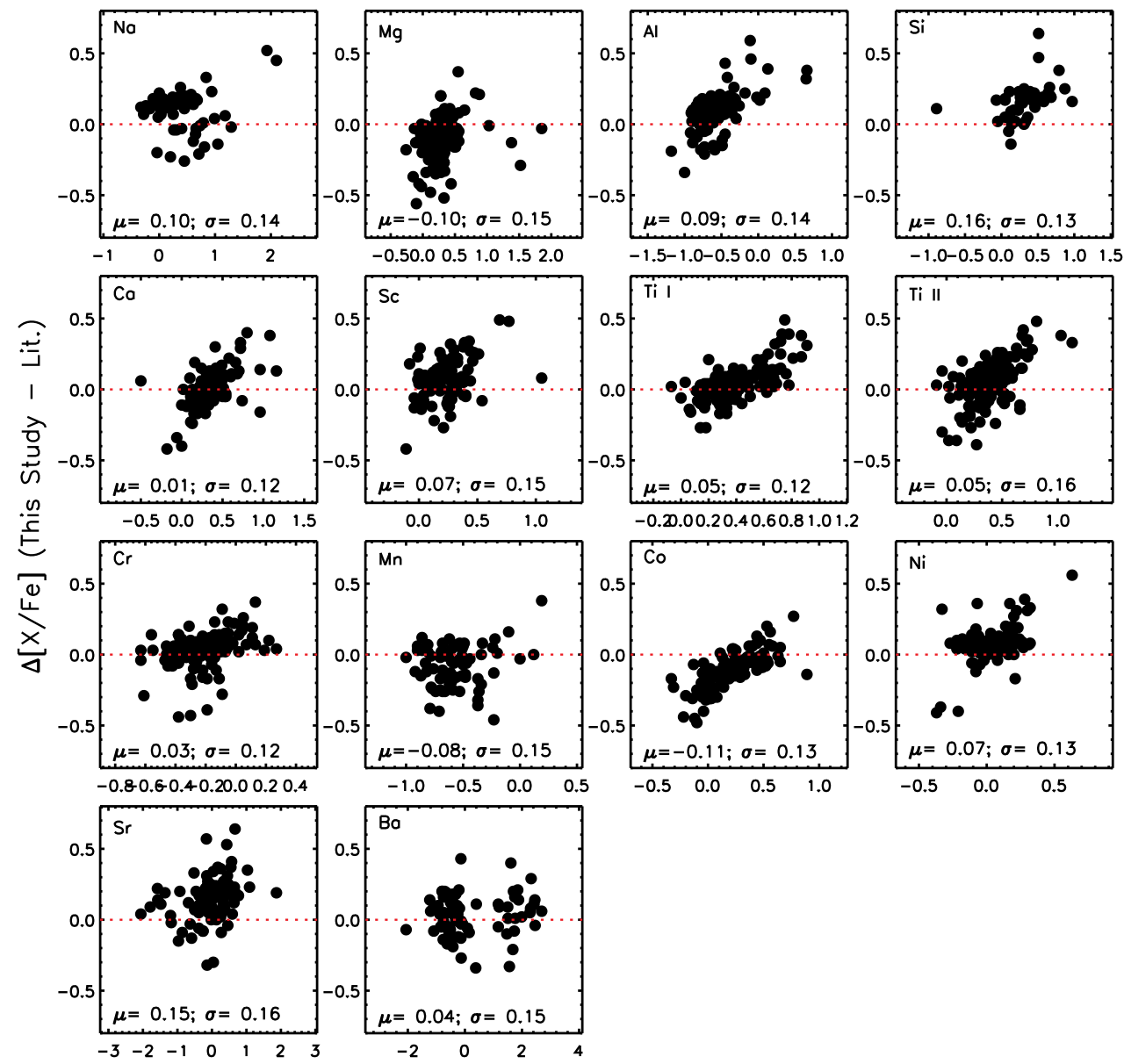

$[\mathrm{X} / \mathrm{Fe}]$ (This Study)

Figure 7. Star-by-star comparison of abundance ratios, $[\mathrm{X} / \mathrm{Fe}]$, for our re-analysis of the literature sample and the original literature values. The numbers at the bottom of each panel are the mean (our analysis - the literature) and the dispersion.

(A color version of this figure is available in the online journal.)

In Figure 7, we compare abundance ratios $[\mathrm{X} / \mathrm{Fe}]$, for $\mathrm{X}=$ $\mathrm{Na}$ to $\mathrm{Ba}$, between the values re-computed in this study and the literature values. For all elements, the average values are in good agreement. The dispersions are comparable to the uncertainties given in Table 3 and, as shown in Section 7.2 below, to the dispersions about the mean trend when plotting $[\mathrm{X} / \mathrm{Fe}]$ versus $[\mathrm{Fe} / \mathrm{H}]$.

In Figures $8-10$, we show the abundance differences $\Delta[\mathrm{X} / \mathrm{Fe}]$ (this study - the literature) for each literature reference or set of references. From these figures, any systematic abundance offsets between our re-analysis and the original literature abundances would be readily seen. Since we have already eliminated the handful of outliers as described above, it is not surprising that our revised abundances are generally in good agreement with the literature values. We shall not seek to understand the reasons for differences in a given element in a particular analysis, except to say that the cause is almost certainly due to the stellar parameters, solar abundance, and/or atomic data. It is reassuring that for the three HES stars with $[\mathrm{Fe} / \mathrm{H}]<-4.5$, our 1D LTE abundances are in good agreement with the published 1D LTE abundances, despite the fact that the published values were based on model atmospheres with appropriate CNO abundances, in contrast to our analysis which assumed scaled solar abundances, but with $[\alpha / \mathrm{Fe}]=+0.40$.

\section{NON-LTE EFFECTS}

Our analysis tools (1D model atmospheres and spectrum synthesis code) assume LTE and, therefore, this analysis is subject to systematic uncertainties from non-LTE as well as 3D (granulation) effects (Asplund 2005). We now offer some comments on the role of non-LTE effects, and refer the reader to work by Asplund and collaborators regarding 3D effects. As discussed extensively in the literature, comparison of the abundances of $\mathrm{Fe}$ from neutral and ionized species provides a check on the presence of departures from LTE and/or the adopted surface gravity. In the event of differences in the abundance from neutral and ionized species, and in the absence of trigonometric distances (e.g., Nissen et al. 1997) and modelinsensitive $T_{\text {eff }}$ measurements, it is difficult to gauge the relative contributions of non-LTE effects or errors in the surface gravity to the abundance discrepancy. Our surface gravities, at least for the program stars, were informed by spectrophotometry and from Balmer-line analysis. That is, both techniques used to derive $T_{\text {eff }}$ required estimates of $\log g($ and $[\mathrm{Fe} / \mathrm{H}])$, and were therefore sensitive to the surface gravity. To explore the degree of non-LTE effects, we shall assume (in this subsection) that any abundance differences between neutral and ionized species reflect non-LTE effects rather than surface-gravity errors. 

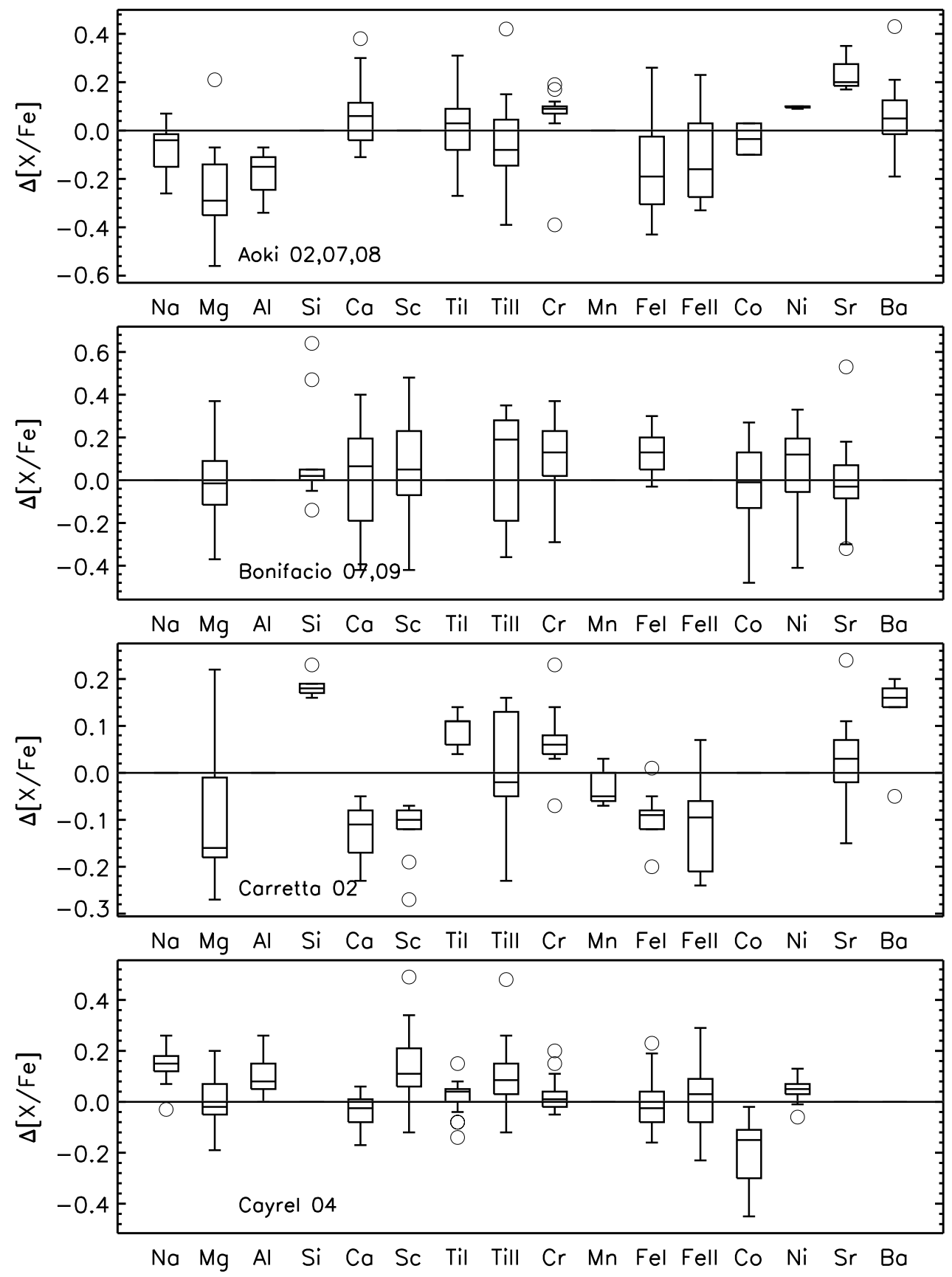

Figure 8. Boxplots illustrating the abundance differences $\Delta[\mathrm{X} / \mathrm{Fe}]$ and $\Delta[\mathrm{Fe} / \mathrm{H}]$ (this study - the literature) for the various literature references. The box defines the interquartile range, the median is identified, the whiskers extend to the maximum (or minimum) or 1.5 times the interquartile range, and circles indicate outliers.

In Figure 11, we plot the difference between the abundance from Fe I and Fe II lines. We only consider stars with two or more Fe II lines. In this figure, we use generalized histograms, in which each data point (i.e., each star) is replaced by a unit Gaussian of width 0.15 dex. The Gaussians are then summed to produce a realistically smoothed histogram. By fitting a Gaussian to this histogram, we can measure the center $(\mu)$ and width of the distribution (FWHM or dispersion, $\sigma$ ). In this figure, we consider (1) all stars, (2) dwarfs ( $\log g>3.0$ ), and (3) giants $(\log g<3.0)$. In all panels, the Gaussian fit to the generalized histogram is centered at $[\mathrm{Fe} \mathrm{I} / \mathrm{H}]-[\mathrm{Fe} \mathrm{II} / \mathrm{H}] \sim-0.04$ dex, and the FWHM and dispersion of the Gaussian fit are 0.27 dex and 0.12 dex, respectively. We note that this dispersion of 0.12 dex is smaller than the average "total error" for Fe I $(0.13$ dex $)$ and Fe II $(0.17 \mathrm{dex})$, added in quadrature $(0.21 \mathrm{dex})$, suggesting that the width of the Gaussian is smaller than that expected from the $\mathrm{Fe}_{\mathrm{I}}$ and $\mathrm{Fe}$ II measurement errors (and assuming that the $\mathrm{Fe}_{\mathrm{I}}$ and Fe II errors are fully independent).

The dominant non-LTE mechanism in late-type metal-poor stars is overionization, and abundances derived from Fe I lines in LTE are expected to be underestimated (e.g., Thévenin \& Idiart 1999; Mashonkina et al. 2011; Bergemann et al. 2012; Lind et al. 2012). Therefore, if non-LTE effects were at play in our sample, we would expect the mean (LTE) value of $[\mathrm{Fe} \mathrm{I} / \mathrm{H}]-[\mathrm{Fe} \mathrm{II} / \mathrm{H}]$ to be negative. For our sample of program stars (red generalized histograms in Figure 11), there is evidence 

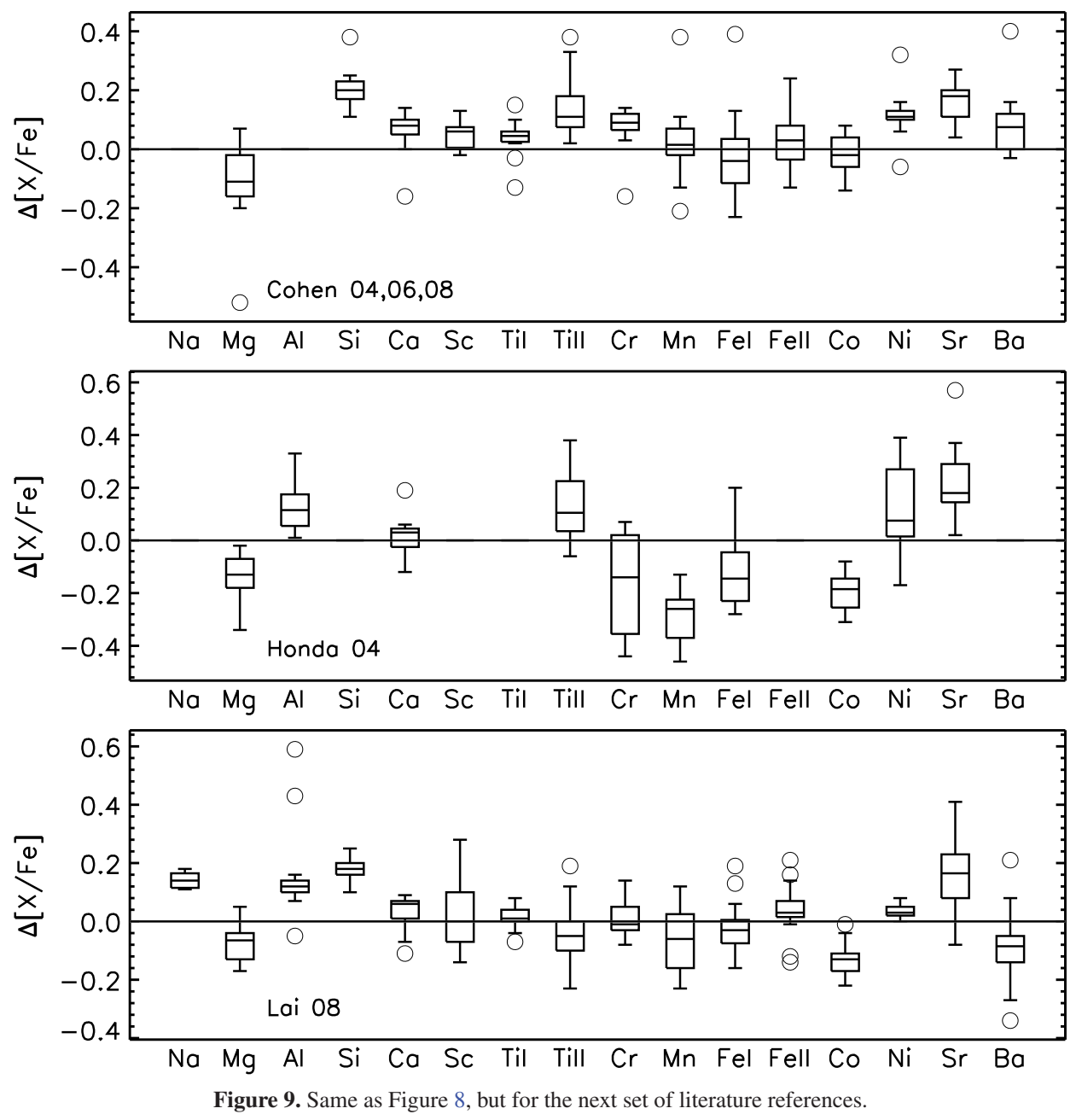

for overionization (our sample is, on average, more metal poor than the full sample, and Lind et al. 2012 show that the degree of overionization is a function of metallicity). However, as noted, the full sample suggests that $\langle[\mathrm{Fe} \mathrm{I} / \mathrm{H}]\rangle \simeq\langle[\mathrm{Fe} \mathrm{II} / \mathrm{H}]\rangle$. We are not suggesting that non-LTE effects are not at play in our sample. Instead, we can only say that the abundances from Fe I and Fe II are, on average, in agreement for both dwarfs and giants for the adopted gravities. The number of stars with $[\mathrm{FeI} / \mathrm{H}]<$ $[\mathrm{Fe} \mathrm{II} / \mathrm{H}]$ is similar to the number with $[\mathrm{Fe} \mathrm{I} / \mathrm{H}]>[\mathrm{Fe} \mathrm{II} / \mathrm{H}]$ for both dwarfs and giants.

Measurements of the Ti abundance from neutral and ionized species permit an alternative view of the possible non-LTE overionization (Bergemann 2011). Given the difference in ionization potentials ( $6.8 \mathrm{eV}$ for $\mathrm{Ti}$ I and $7.9 \mathrm{eV}$ for $\mathrm{Fe} \mathrm{I}$ ), one might naively expect overionization to affect Ti to a larger degree than for Fe. In Figure 12, we plot the difference between the abundance from Ti I and Ti II lines. We again use generalized histograms, in which each data point is replaced by a unit Gaussian of width 0.15 dex. Only stars with two or more Ti I and Ti II lines are considered; we fit a Gaussian to the generalized histogram to quantify the center $(\mu)$ and width (FWHM or dispersion, $\sigma$ ). In this figure, we consider (1) all stars, (2) dwarfs $(\log g>3.0)$, and $(3)$ giants $(\log g<3.0)$. For all stars and for the giant sample, the Gaussians are centered near 0 . However, for the dwarfs, the Gaussian is centered at +0.19 dex. This indicates that for the dwarfs, the abundance from Ti I exceeds that from Ti II, a result not seen for Fe. Such a discrepancy between the abundance from neutral and ionized species has the opposite sign compared with that expected from non-LTE overionization. The largest dispersion is 0.16 dex (for the dwarfs), and this value is smaller than the "total error" for Ti I $(0.16 \mathrm{dex})$ and Ti II $(0.16 \mathrm{dex})$, added in quadrature $(0.23 \mathrm{dex})$. This again suggests that the width of the Gaussian is smaller than that expected from the Ti I and Ti II measurement errors.

Non-LTE effects can also manifest as trends between the abundance from $\mathrm{Fe} \mathrm{I}$ lines and the lower excitation potential $(\chi)$. Other studies of metal-poor stars (e.g., Cayrel et al. 2004; Cohen et al. 2008; Lai et al. 2008) find a negative trend between the Fe I abundance and $\chi$, which could be due to systematic errors in the $\log g f$ values, non-LTE effects, or temperature errors. While revision of the temperature scale could alleviate this trend, the magnitude of the required correction $(\mathrm{a} \sim 200 \mathrm{~K}$ reduction in $T_{\text {eff }}$ for most cases, but a considerably larger reduction in $T_{\text {eff }}$ for several stars) exceeds our estimate of the uncertainty in $T_{\text {eff }}$. We also find such a trend for both the program stars and the literature stars. When considering all lines, the program stars show an average trend of $-0.04 \mathrm{dex} \mathrm{eV}^{-1}(\sigma=0.05)$; the literature sample, as re-analyzed here, shows an identical average trend and dispersion. (When considering the dwarf and giant samples separately, there is no difference between the two populations.)

Our adopted $T_{\text {eff }}$ values do not rely on the excitation balance of Fe I lines. Hosford et al. (2010) and Lind et al. (2012) have demonstrated that excitation temperatures are susceptible to 

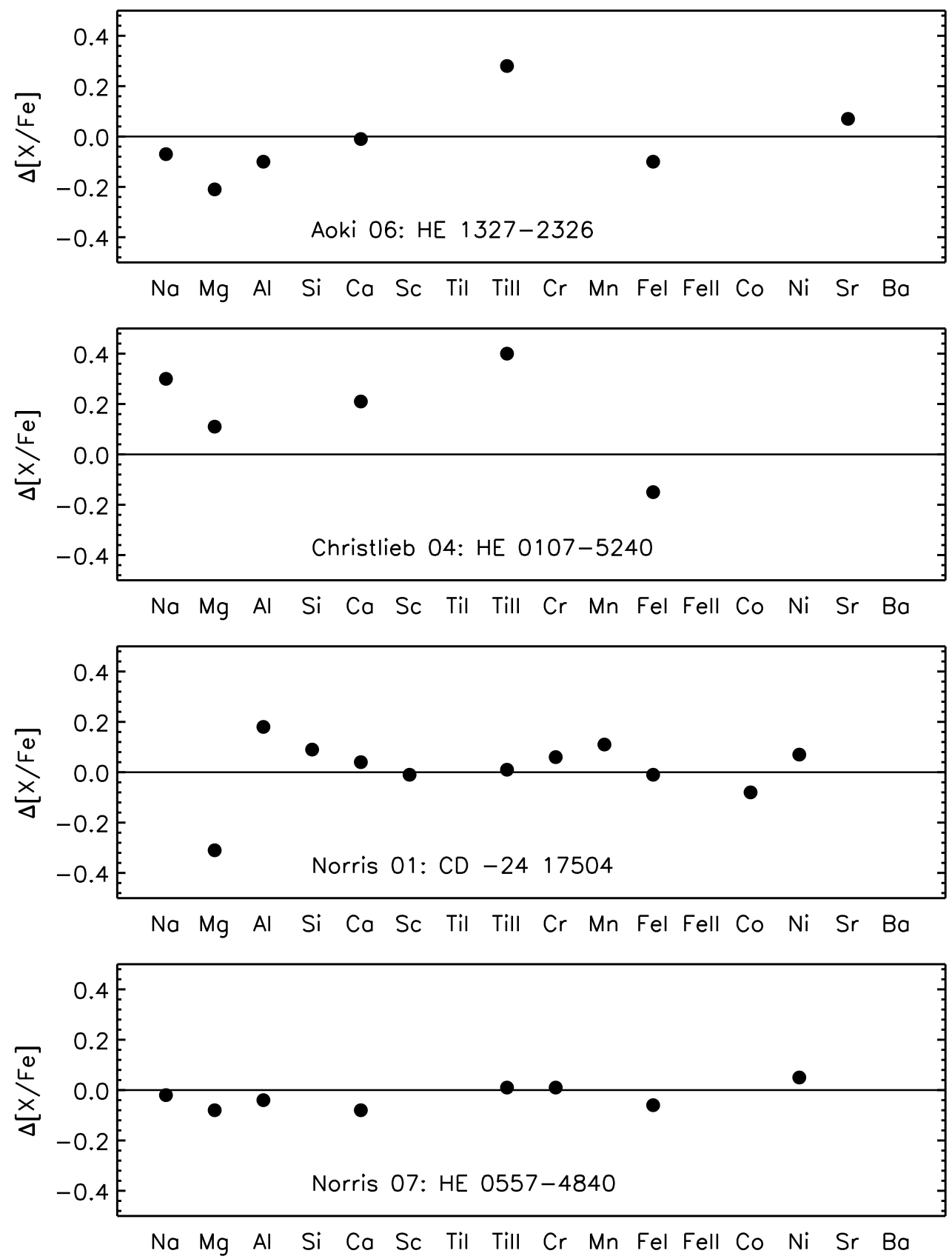

Figure 10. Abundance differences $\Delta[\mathrm{X} / \mathrm{Fe}]$ and $\Delta[\mathrm{Fe} / \mathrm{H}]$ (this study - the literature) for individual stars.

non-LTE effects. Asplund \& García Pérez (2001) showed that IRFM $T_{\text {eff }}$ values are little dependent on $3 \mathrm{D}$ effects, although a more systematic investigation would be welcome. Had we employed excitation temperatures (and their corresponding surface gravities and microturbulent velocities), we would have obtained different metallicities and abundance ratios $[\mathrm{X} / \mathrm{Fe}]$. We find that excluding lines with $\chi<1.2 \mathrm{eV}$ decreases the average trend between excitation potential and $\mathrm{Fe}$ I abundance, as seen in previous studies of metal-poor stars. When considering only lines with $\chi \geqslant 1.2 \mathrm{eV}$, the average trends are $0.01 \mathrm{dex} \mathrm{eV}^{-1}$ $(\sigma=0.20)$ and $-0.03 \mathrm{dex} \mathrm{eV}^{-1}(\sigma=0.11)$ for the program stars and literature stars, respectively. For the program stars, had we included only those lines with $\chi>1.2 \mathrm{eV}$, the average $[\mathrm{Fe} / \mathrm{H}]$ would be lower by only $0.01 \pm 0.01 \mathrm{dex}(\sigma=0.06 \mathrm{dex})$. A more detailed assessment of the role and magnitude of non-
LTE effects is beyond the scope of the present paper, though we shall touch upon the matter again at several points in the subsections that follow.

\section{RESULTS}

\subsection{CEMP Objects}

We adopted the Aoki et al. (2007) definition for CEMP stars, which accounts for nucleosynthesis and mixing in evolved giants, namely, (1) $[\mathrm{C} / \mathrm{Fe}] \geqslant+0.70$, for $\log \left(L / L_{\odot}\right) \leqslant 2.3$, and (2) $[\mathrm{C} / \mathrm{Fe}] \geqslant+3.0-\log \left(L / L_{\odot}\right)$, for $\log \left(L / L_{\odot}\right)>2.3$. The original definition proposed by Beers \& Christlieb (2005) is $[\mathrm{C} / \mathrm{Fe}] \geqslant+1.0$. In Figure 13 , we plot $[\mathrm{C} / \mathrm{Fe}]$ versus $\log \left(L / L_{\odot}\right)$, showing both CEMP definitions. We refer the reader to Paper III for more discussion of the CEMP fraction (and the metallicity 

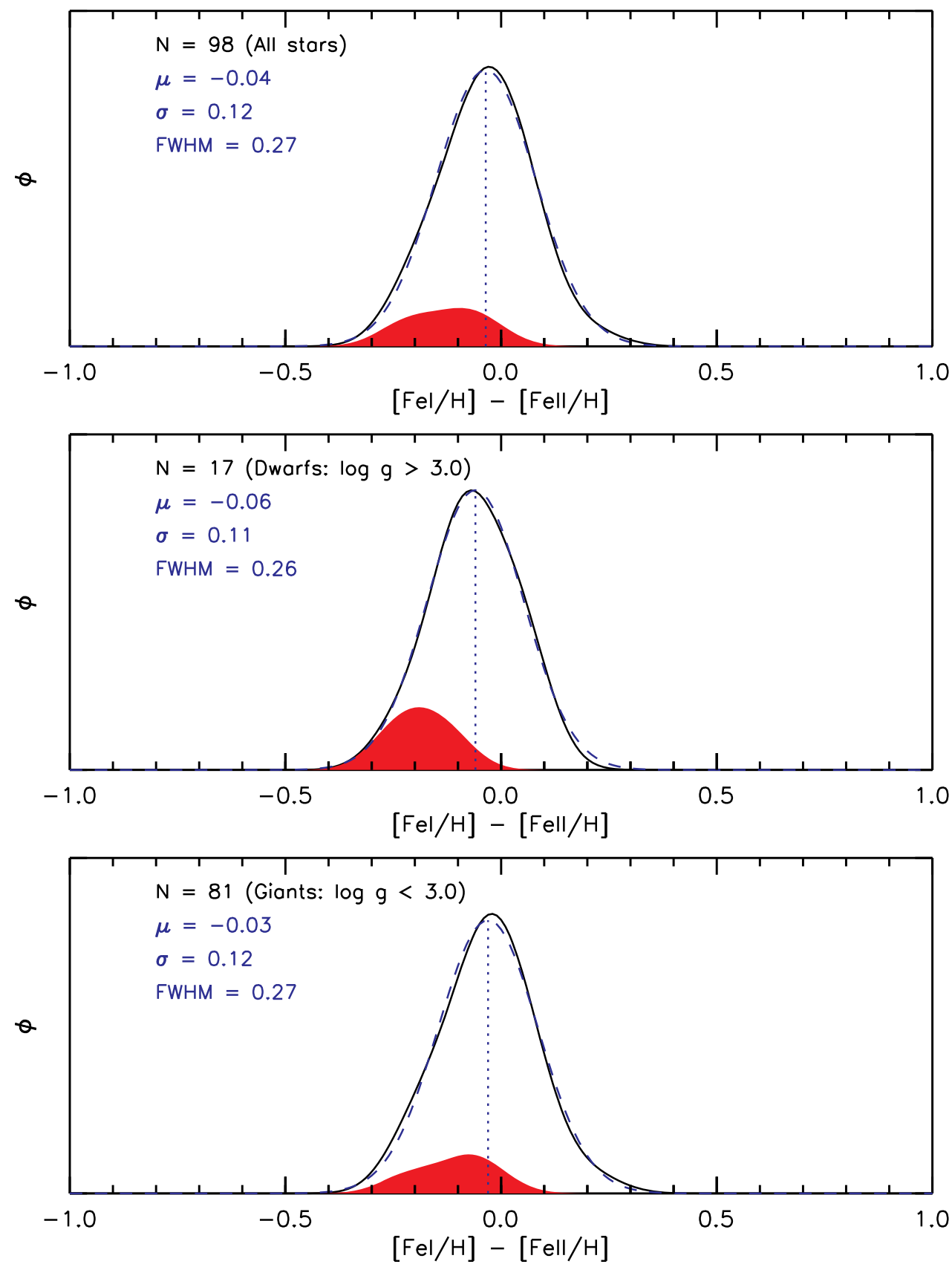

Figure 11. Generalized histograms showing the abundance difference $[\mathrm{Fe} / \mathrm{H}]-[\mathrm{Fe} \mathrm{II} / \mathrm{H}]$ for $\mathrm{N} \geqslant 2$ lines and $[\mathrm{Fe} / \mathrm{H}] \leqslant-2.5$. The red histograms represent the relevant subset of our 38 program stars. The upper panel shows all stars, the middle panel only dwarfs $(\log g>3.0)$, and the lower panel only giants $(\log g<3.0)$. In each panel we show the number of stars and the $\mu$ and $\sigma$ for the Gaussian $\left(a e^{-(x-\mu)^{2} / 2 \sigma^{2}}\right)$ fit (dashed blue line) to the data, as well as the FWHM.

(A color version of this figure is available in the online journal.)

distribution function) at lowest metallicity. (Throughout the present paper, we use CEMP and C-rich interchangeably.) None of our program stars are C-normal objects. This is likely due to selection biases and that we could only obtain $[\mathrm{C} / \mathrm{Fe}]$ limits for many program stars.

\subsection{Abundance Trends $[\mathrm{X} / \mathrm{Fe}]$ versus $[\mathrm{Fe} / \mathrm{H}]$}

In Figure 14, we plot abundance ratios $[\mathrm{X} / \mathrm{Fe}]$ versus $[\mathrm{Fe} / \mathrm{H}]$ for the 38 program stars. The CEMP stars are marked in red in each panel. The green line in this figure represents the predictions from the Galactic chemical enrichment models of Kobayashi et al. (2006), which will also be discussed in Sections 7.5 and 7.7. For all elements, the abundance dispersion exceeds the measurement uncertainty. In particular, $\mathrm{Na}, \mathrm{Sr}$, and $\mathrm{Ba}$ exhibit very large dispersions, as do $\mathrm{C}$ and $\mathrm{N}$. Before we consider the complete sample (program stars + literature stars), we note that the abundances of $\mathrm{Mg}$ and $\mathrm{Si}$ appear to be lower than the canonical halo value of $[\alpha / \mathrm{Fe}]=+0.4$. Furthermore, for a given element, the outliers are often, but not always, CEMP objects. However, for many of our stars we could only obtain upper limits for the $\mathrm{C}$ abundance. It would be interesting to re-assess whether the abundance outliers are always CEMP objects by either (1) restricting the sample to those stars with 

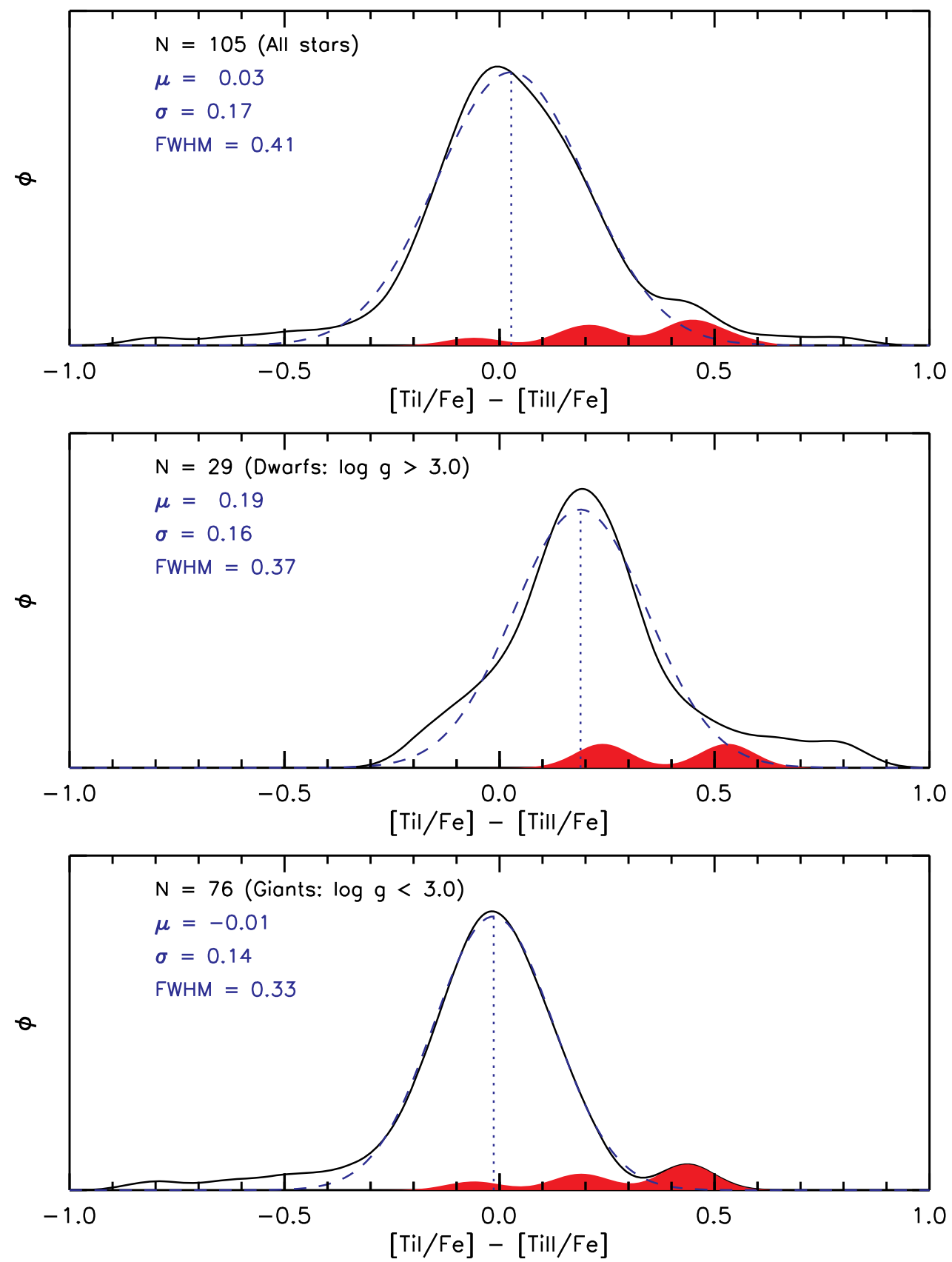

Figure 12. Same as Figure 11, but for [Ti I/Fe] - [Ti II/Fe].

(A color version of this figure is available in the online journal.)

$\mathrm{C}$ measurements and/or (2) obtaining higher-quality spectra to convert upper limits for $\mathrm{C}$ into detections.

For each element from $\mathrm{C}$ to $\mathrm{Ba}$, we plot the abundance ratio $[\mathrm{X} / \mathrm{Fe}]$ versus $[\mathrm{Fe} / \mathrm{H}]$ for the combined sample, i.e., program stars and literature stars (Figures 15-30). In all figures, the left panels show only the dwarf stars $(\log g>3.0)$, and the right panels show only the giant stars $(\log g<3.0)$. In Figures $17-30$, we determine the linear fit to the data in the following manner. First, we exclude all CEMP stars from the fit. Second, we determine the linear fit and measure the dispersion about the mean trend. Third, we eliminate $2 \sigma$ outliers from the fit. Fourth, we re-determine the linear fit and show in the plots (1) the slope of the fit and its associated uncertainty, (2) the dispersion about the fit, and (3) the mean abundance, $[\mathrm{X} / \mathrm{Fe}]$, and the standard deviation. The reason for excluding C-rich objects and $2 \sigma$ outliers was that we were seeking to (1) identify a "normal" population of metal-poor stars and (2) characterize the mean trend between $[\mathrm{X} / \mathrm{Fe}]$ and $[\mathrm{Fe} / \mathrm{H}]$ for this "normal" population. We emphasize that while CEMP objects and $2 \sigma$ outliers were not included in determining the best fits to the data, these objects are included in all plots.

Our motivation for attempting to define a normal population comes from the FIRST STARs analyses (Cayrel et al. 2004; Spite et al. 2005; François et al. 2007; Bonifacio et al. 2009). As mentioned, these studies (along with Arnone et al. 2005) found extremely small scatter, which is likely due in part to 


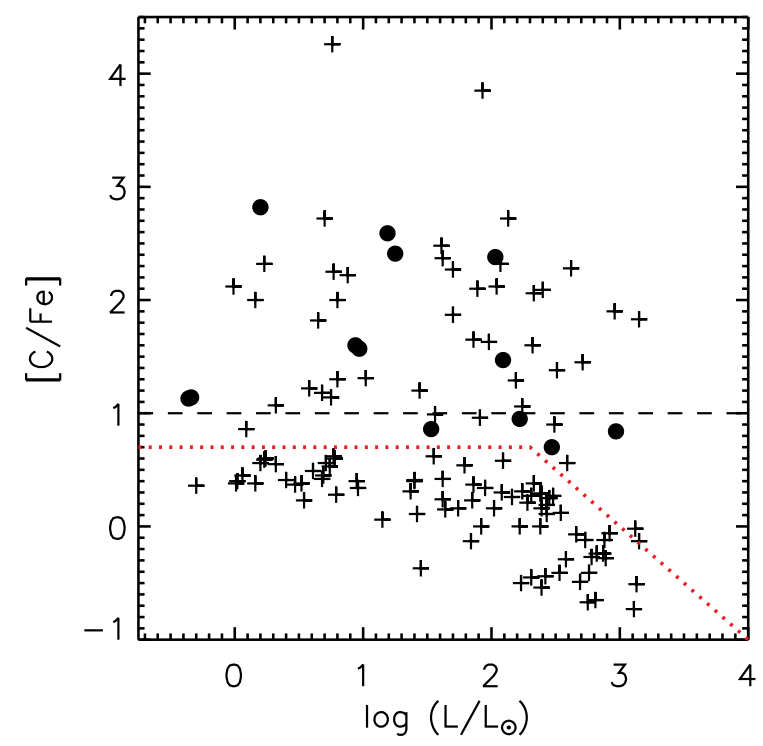

Figure 13. $[\mathrm{C} / \mathrm{Fe}]$ vs. $\log \left(L / L_{\odot}\right)$ for the program stars (circles) and the literature sample (plus signs). (Only detections are plotted.) The dashed line shows the Beers \& Christlieb (2005) CEMP definition, $[\mathrm{C} / \mathrm{Fe}]=+1.0$, while the red dotted line shows the Aoki et al. (2007) CEMP definition.

(A color version of this figure is available in the online journal.) the high-quality data and analysis, and to the fact that their samples included only two CEMP giants (CS 22949-037 and CS 22892-052) and one CEMP dwarf (CS 29527-015). Thus, they are "biased against carbon-rich objects and cannot be used to constrain the full dispersion of carbon abundances at the lowest metallicities" (Cayrel et al. 2004, p. 10). As identified in the literature, and confirmed in the present series, CEMP stars often have anomalous abundance ratios for elements other than C. However, it has also become evident that some C-normal stars show peculiar abundances for other elements (e.g., Cohen et al. 2008). Therefore, when searching for a "normal" population of metal-poor stars, we eliminate CEMP objects as well as $2 \sigma$ outliers from the fit, but retain them in the plots.

In these figures, we also present contour plots illustrating the density in the $[\mathrm{X} / \mathrm{Fe}]$ versus $[\mathrm{Fe} / \mathrm{H}]$ plane. We consider (1) C-normal dwarfs, (2) CEMP dwarfs, (3) C-normal giants, and (4) CEMP giants. For each sample, we represent a given data point with a two-dimensional Gaussian, for which the FWHM in the $[\mathrm{Fe} / \mathrm{H}]$ and $[\mathrm{X} / \mathrm{Fe}]$ directions corresponds to our estimates of the typical measurement uncertainties for $[\mathrm{Fe} / \mathrm{H}]$ and $[\mathrm{X} / \mathrm{Fe}]$, respectively. The height of each Gaussian is set to 1.0. The Gaussians are then summed and a contour plot is generated. In the event that a given panel has $N$ data points with a single value of $[\mathrm{Fe} / \mathrm{H}]$ and $[\mathrm{X} / \mathrm{Fe}]$, the contour would have a
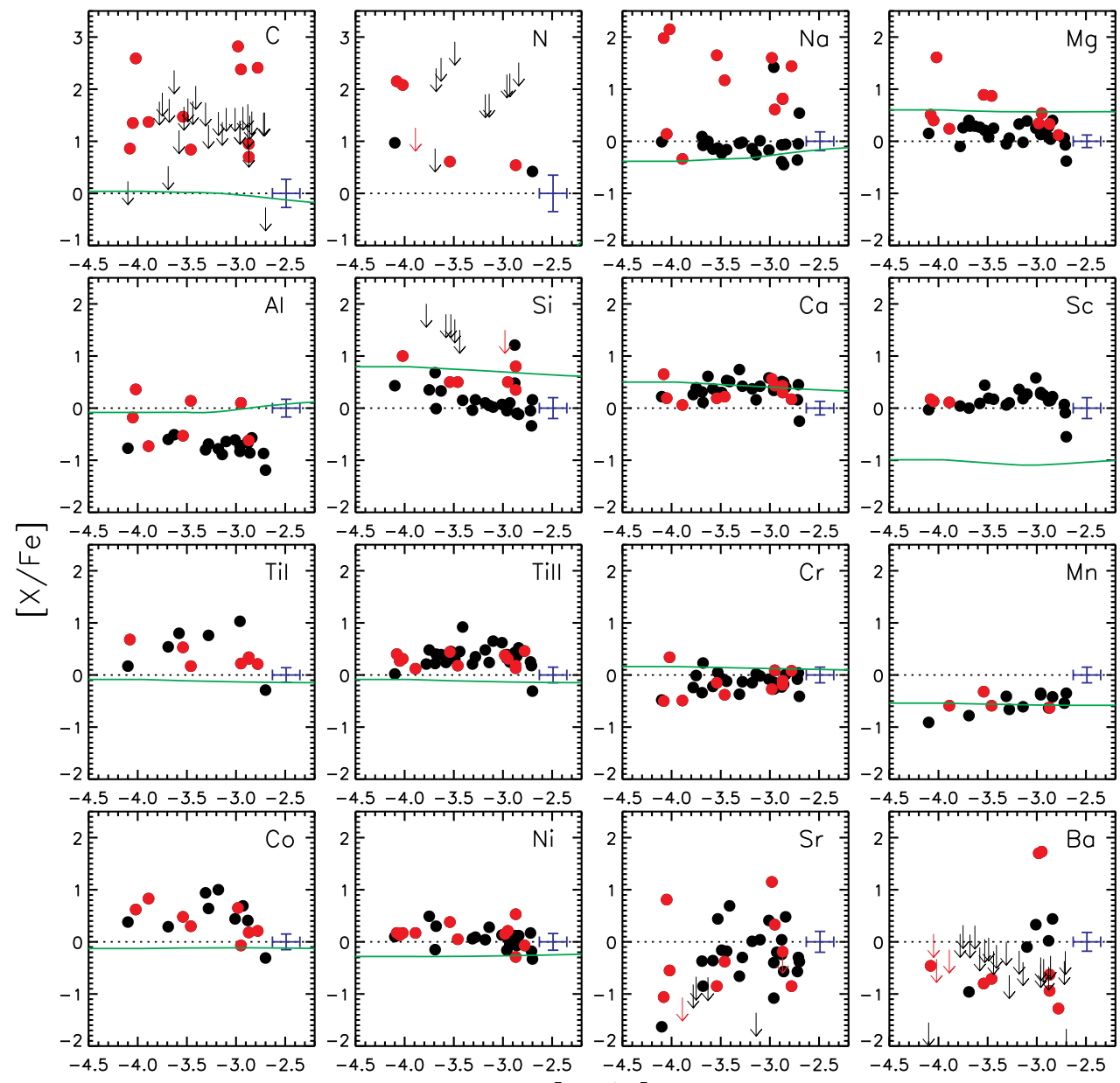

$-4.5-4.0-3.5-3.0-2.5$
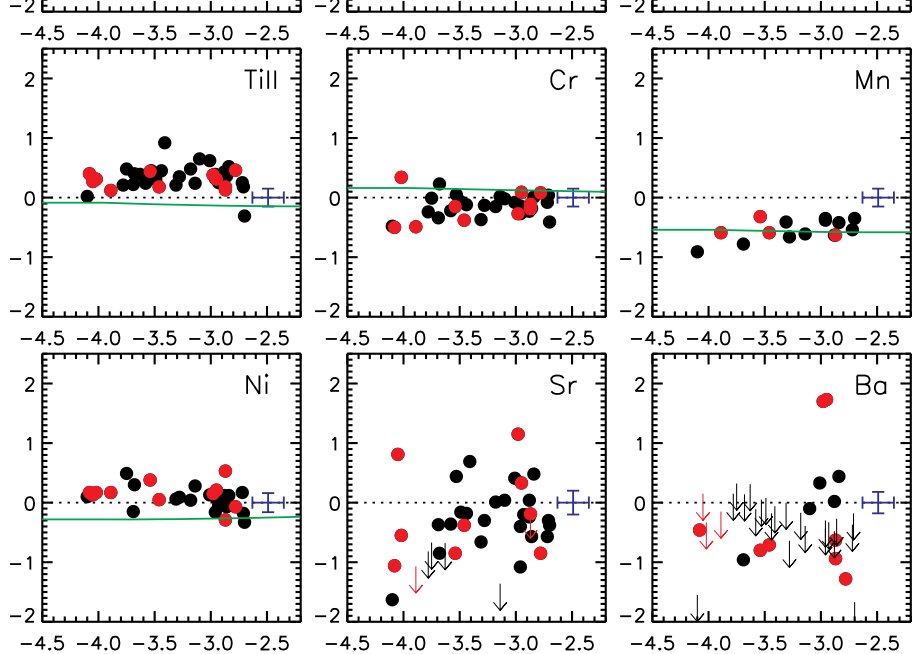

$[\mathrm{Fe} / \mathrm{H}]$

Figure 14. $[\mathrm{X} / \mathrm{Fe}]$ vs. $[\mathrm{Fe} / \mathrm{H}]$ for the program stars. Stars with $[\mathrm{C} / \mathrm{Fe}] \geqslant+1.0$ are marked in red. The solid green line represents the predictions from Kobayashi et al. (2006). A representative error bar is shown in each panel.

(A color version of this figure is available in the online journal.) 


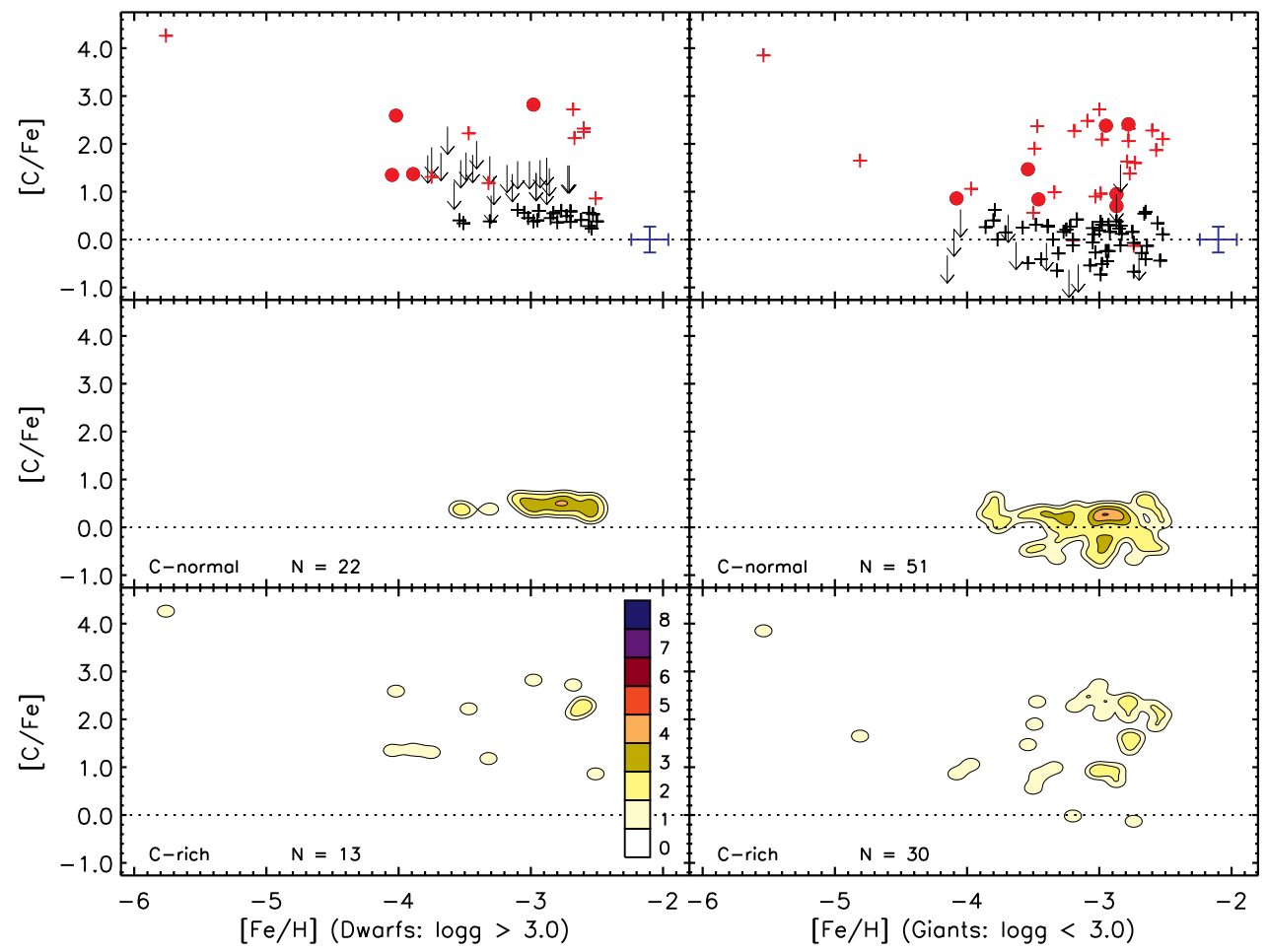

Figure 15. $[\mathrm{C} / \mathrm{Fe}]$ vs. $[\mathrm{Fe} / \mathrm{H}]$ for stars with $[\mathrm{Fe} / \mathrm{H}] \leqslant-2.5$. The left panels present only dwarf stars $(\log g>3.0)$ and the right panels only giants $(\log g<3.0)$, respectively. In the upper panels, our program stars are shown as circles, and the literature sample as plus signs. Red symbols denote C-rich objects (i.e., CEMP stars), while black symbols represent C-normal stars. In the top panels we plot a representative uncertainty (the average "total error" for the program stars). In the middle panels we present contour plots of the data for C-normal dwarfs and giants, while the lower panels show contour plots for C-rich dwarfs and giants.

(A color version of this figure is available in the online journal.)

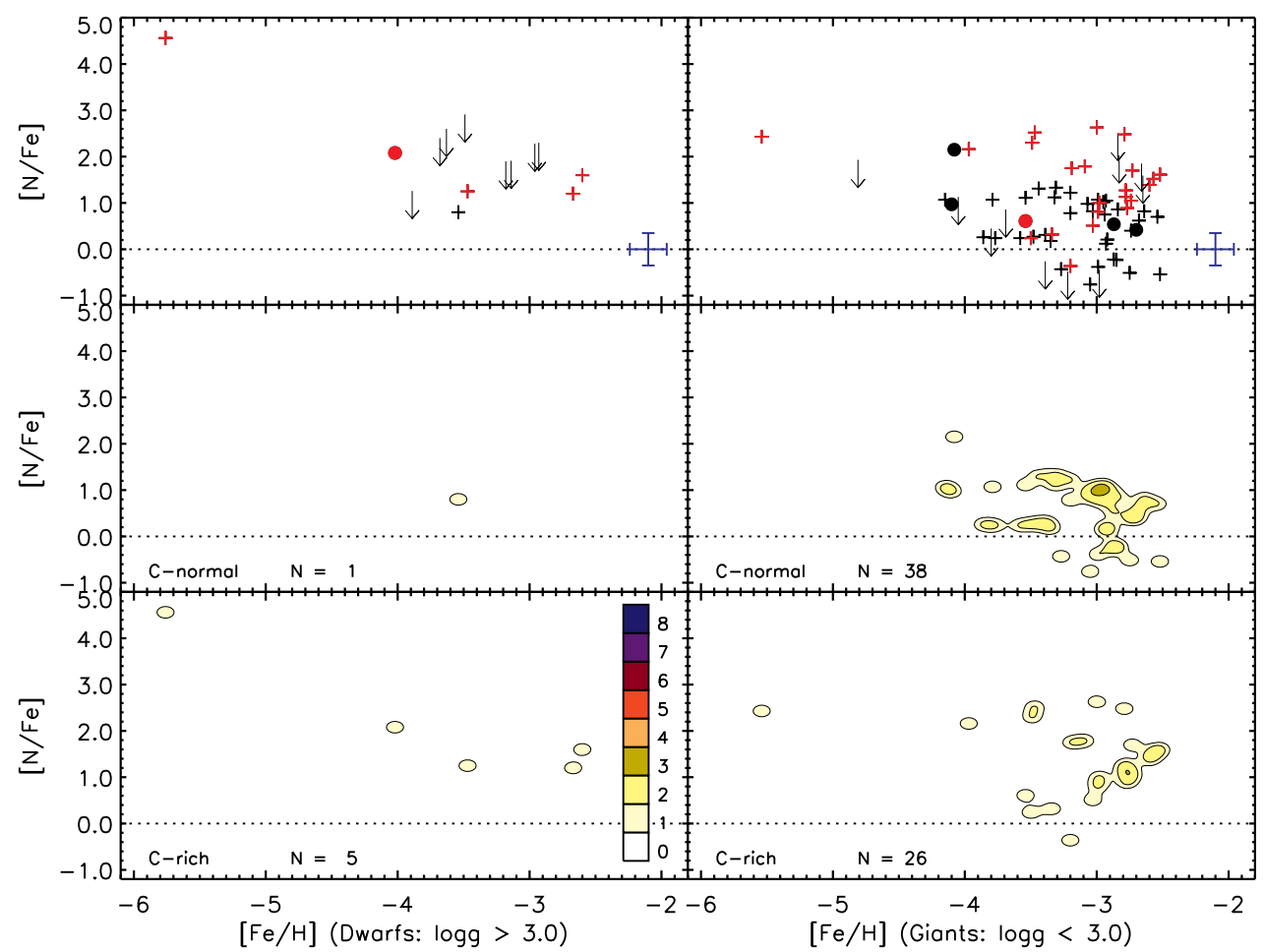

Figure 16. Same as Figure 15, but for $[\mathrm{N} / \mathrm{Fe}]$. Here the red symbols mark objects with $[\mathrm{N} / \mathrm{Fe}] \geqslant+1.0$.

(A color version of this figure is available in the online journal.) 


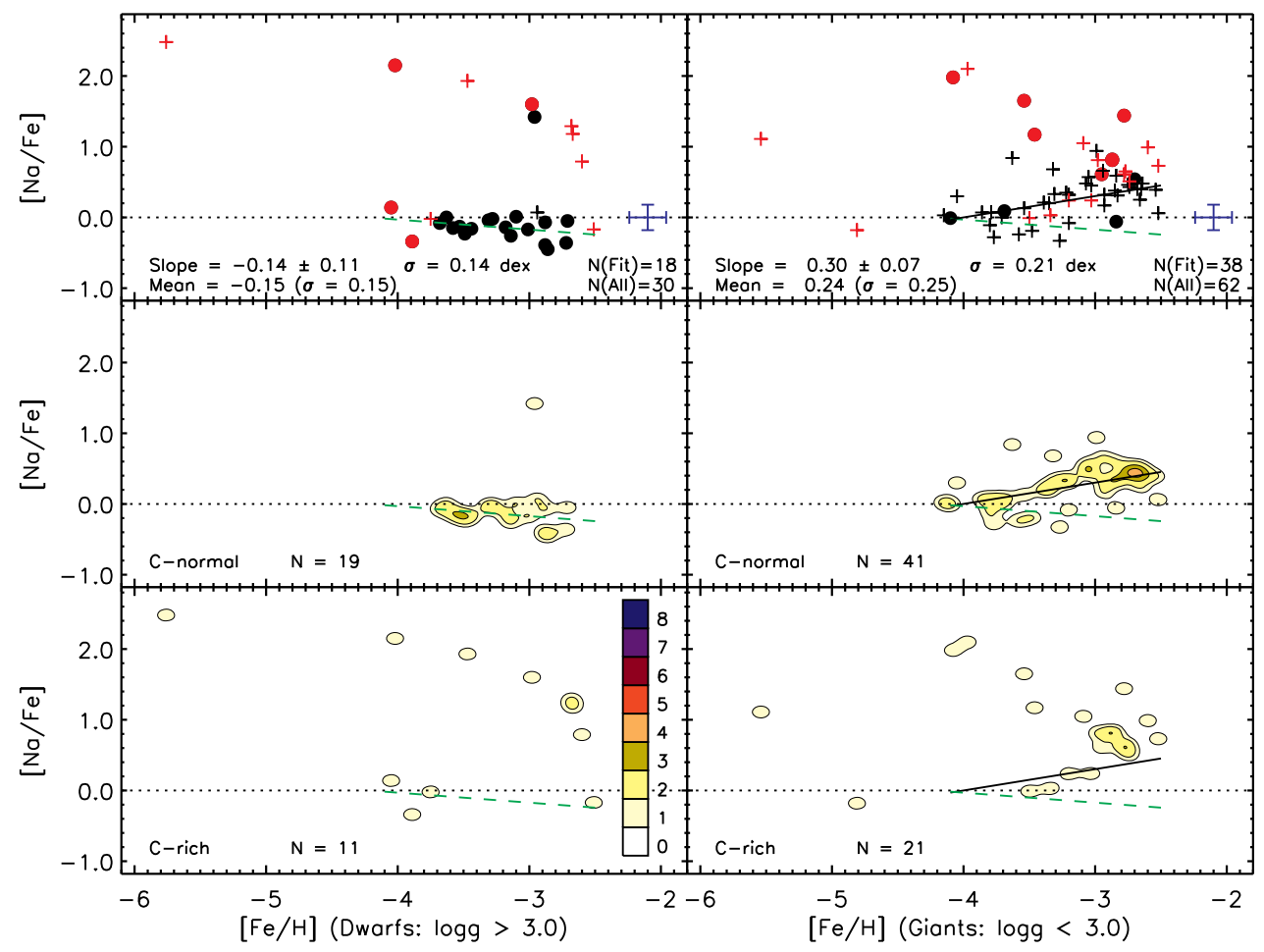

Figure 17. Same as Figure 15, but for $[\mathrm{Na} / \mathrm{Fe}]$. In the left panels, we present the linear fit to the dwarf data (dashed green line), excluding CEMP objects and $2 \sigma$ outliers. The slope (and associated error) of this fit are shown along with the dispersion about the best fit. The right panels contain the linear fit to the giant data (black solid line) again excluding CEMP objects and $2 \sigma$ outliers. In the right panels, we overplot the linear fit to the dwarf sample.

(A color version of this figure is available in the online journal.)

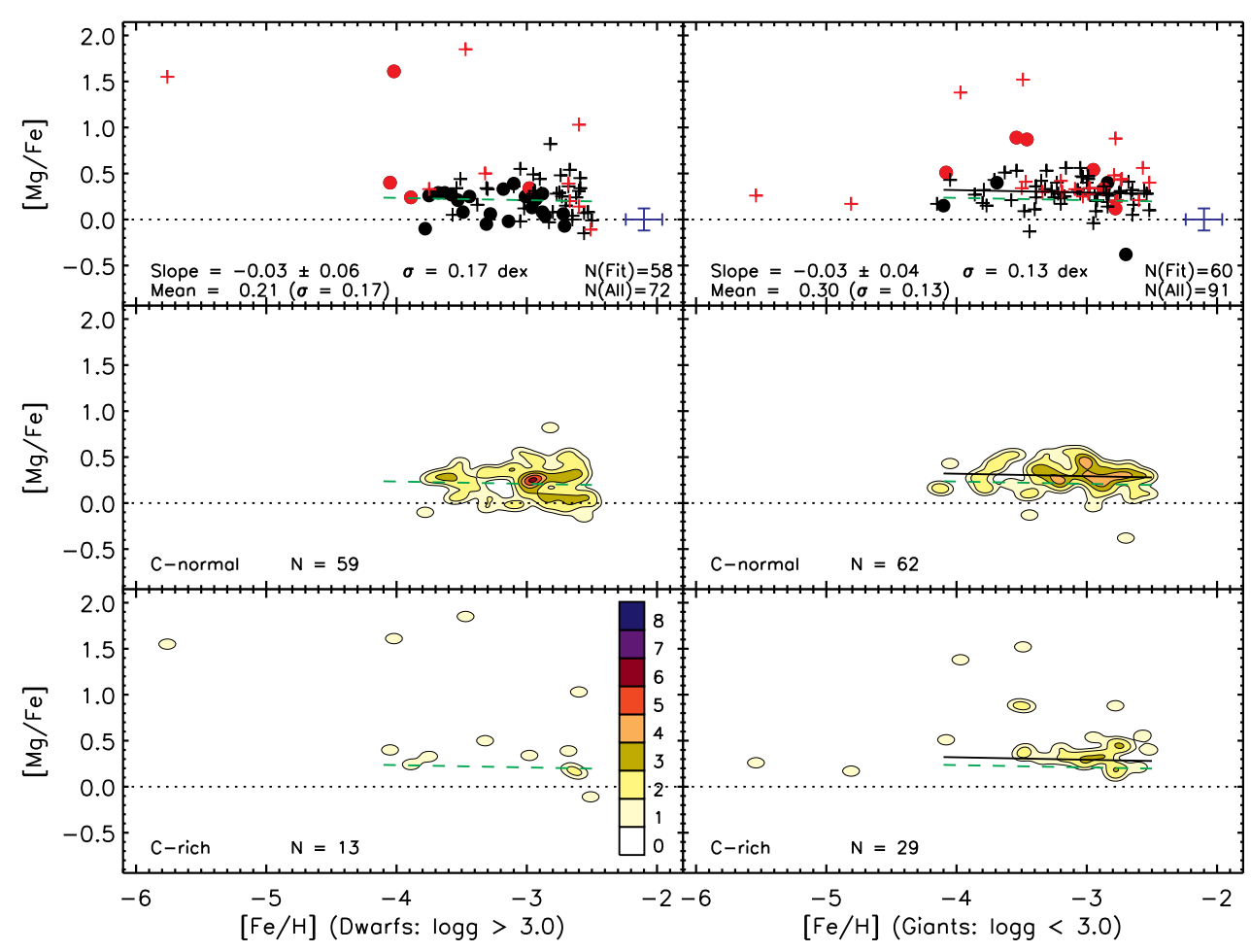

Figure 18. Same as Figure 17, but for $[\mathrm{Mg} / \mathrm{Fe}]$.

(A color version of this figure is available in the online journal.) 


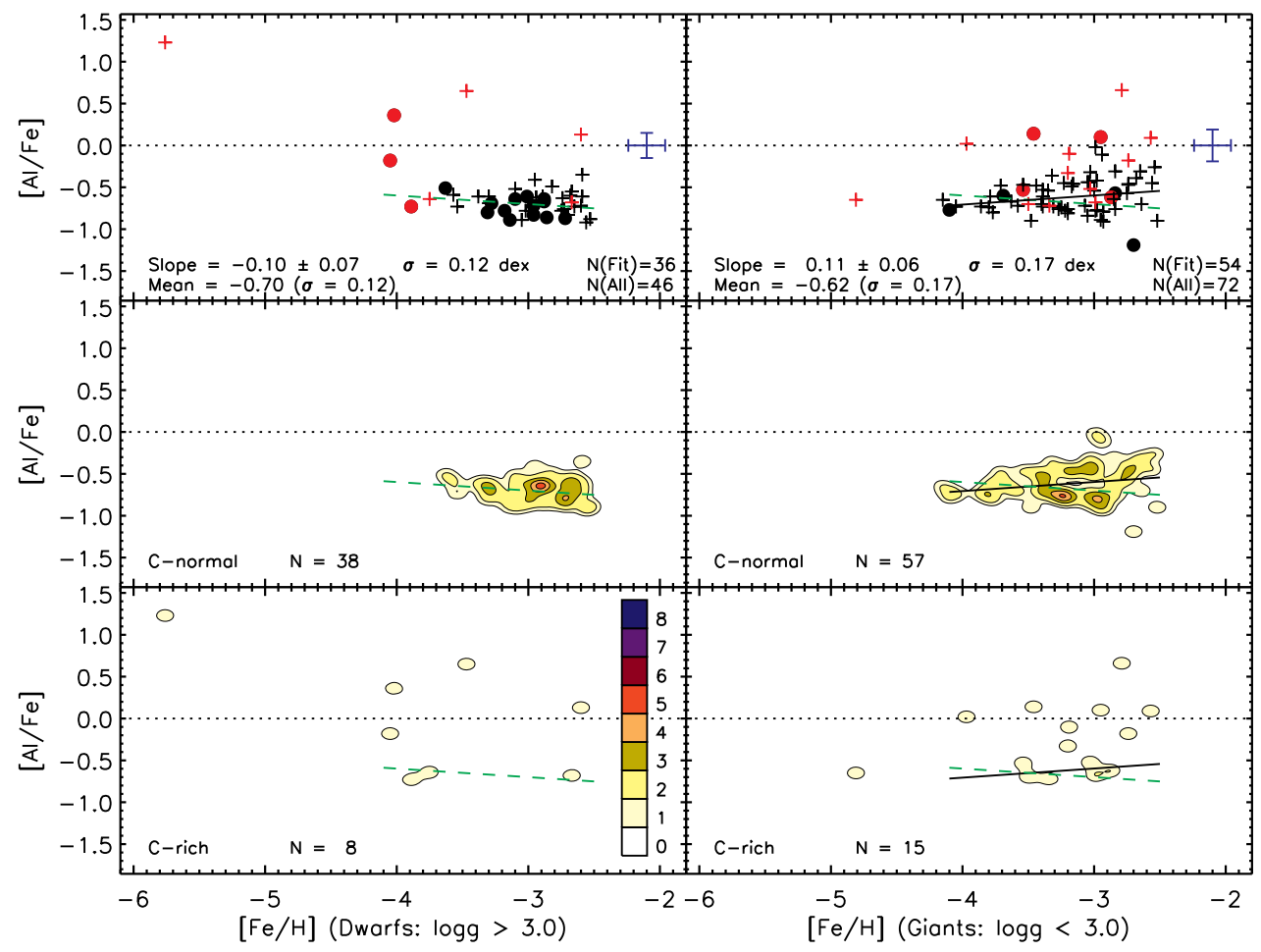

Figure 19. Same as Figure 17, but for $[\mathrm{Al} / \mathrm{Fe}]$.

(A color version of this figure is available in the online journal.)

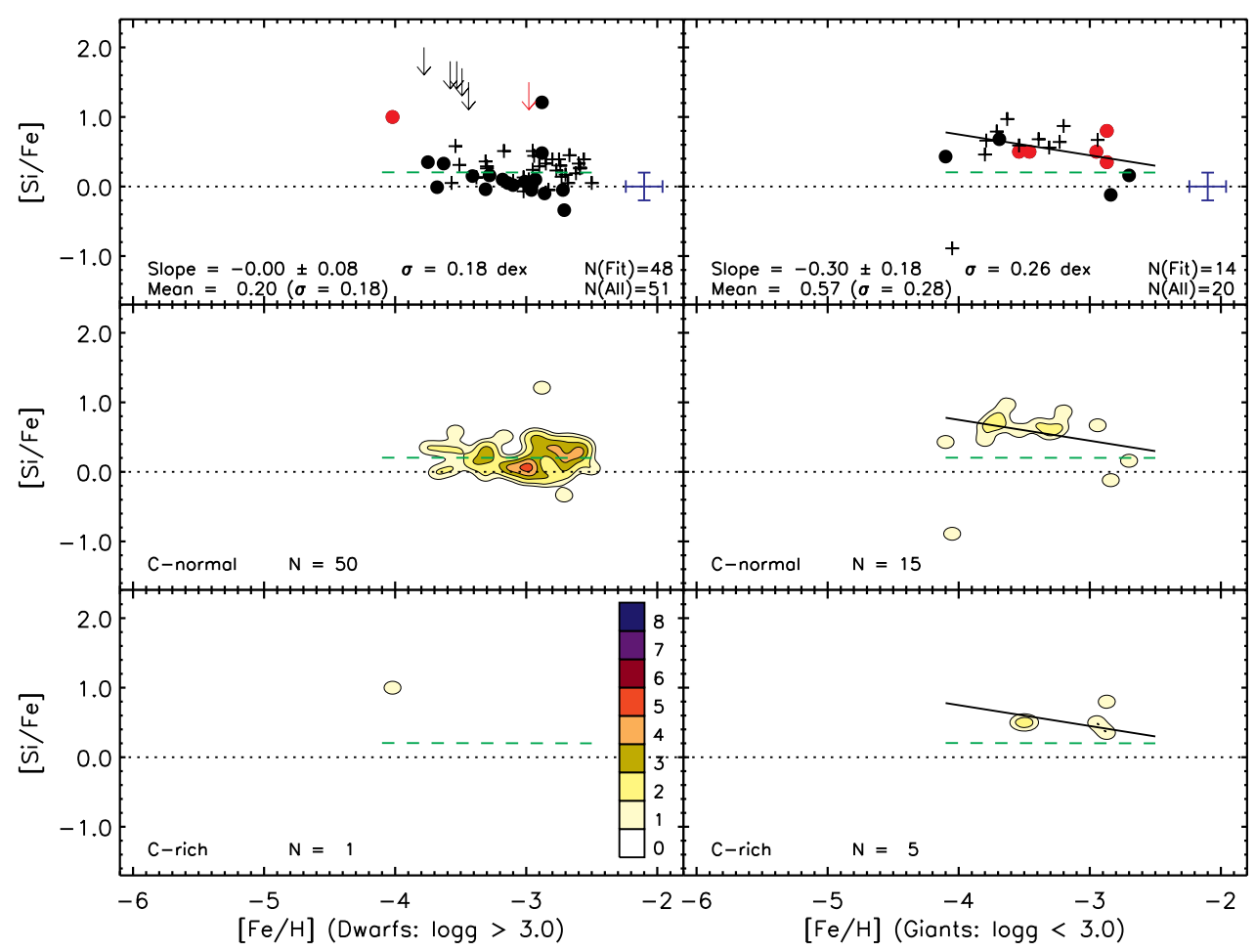

Figure 20. Same as Figure 17, but for $[\mathrm{Si} / \mathrm{Fe}]$.

(A color version of this figure is available in the online journal.) 


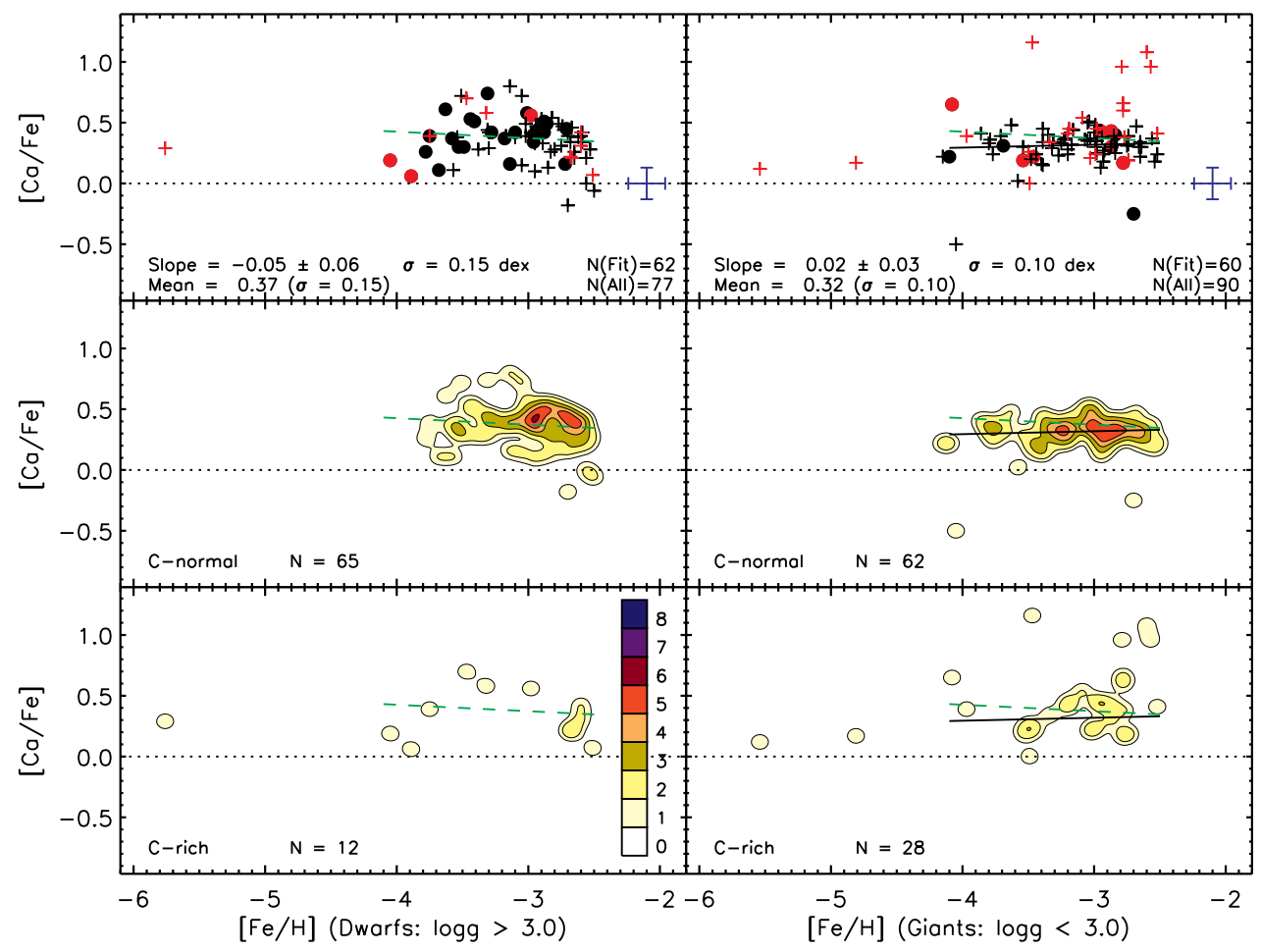

Figure 21. Same as Figure 17, but for $[\mathrm{Ca} / \mathrm{Fe}]$

(A color version of this figure is available in the online journal.)

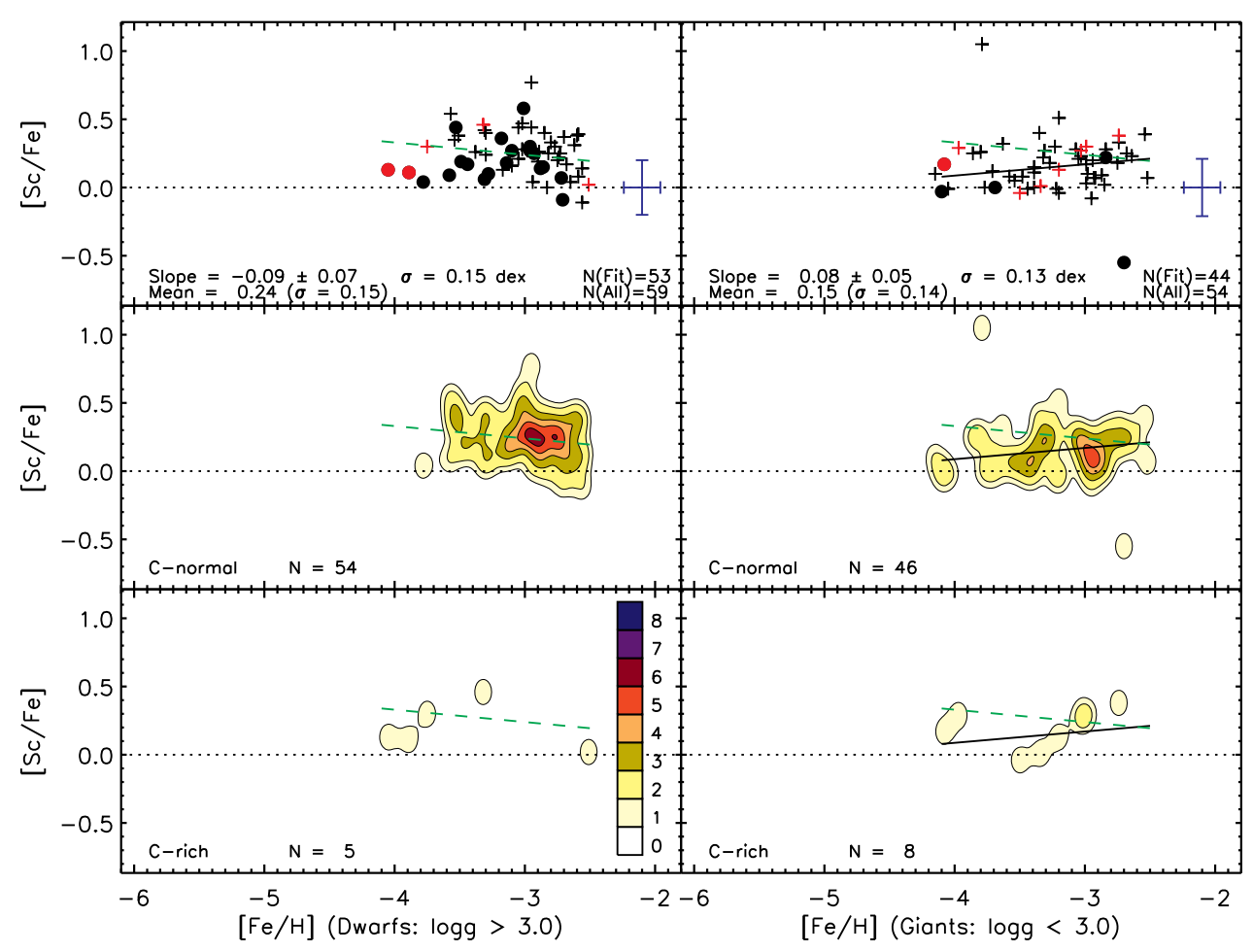

Figure 22. Same as Figure 17, but for $[\mathrm{Sc} / \mathrm{Fe}]$.

(A color version of this figure is available in the online journal.) 


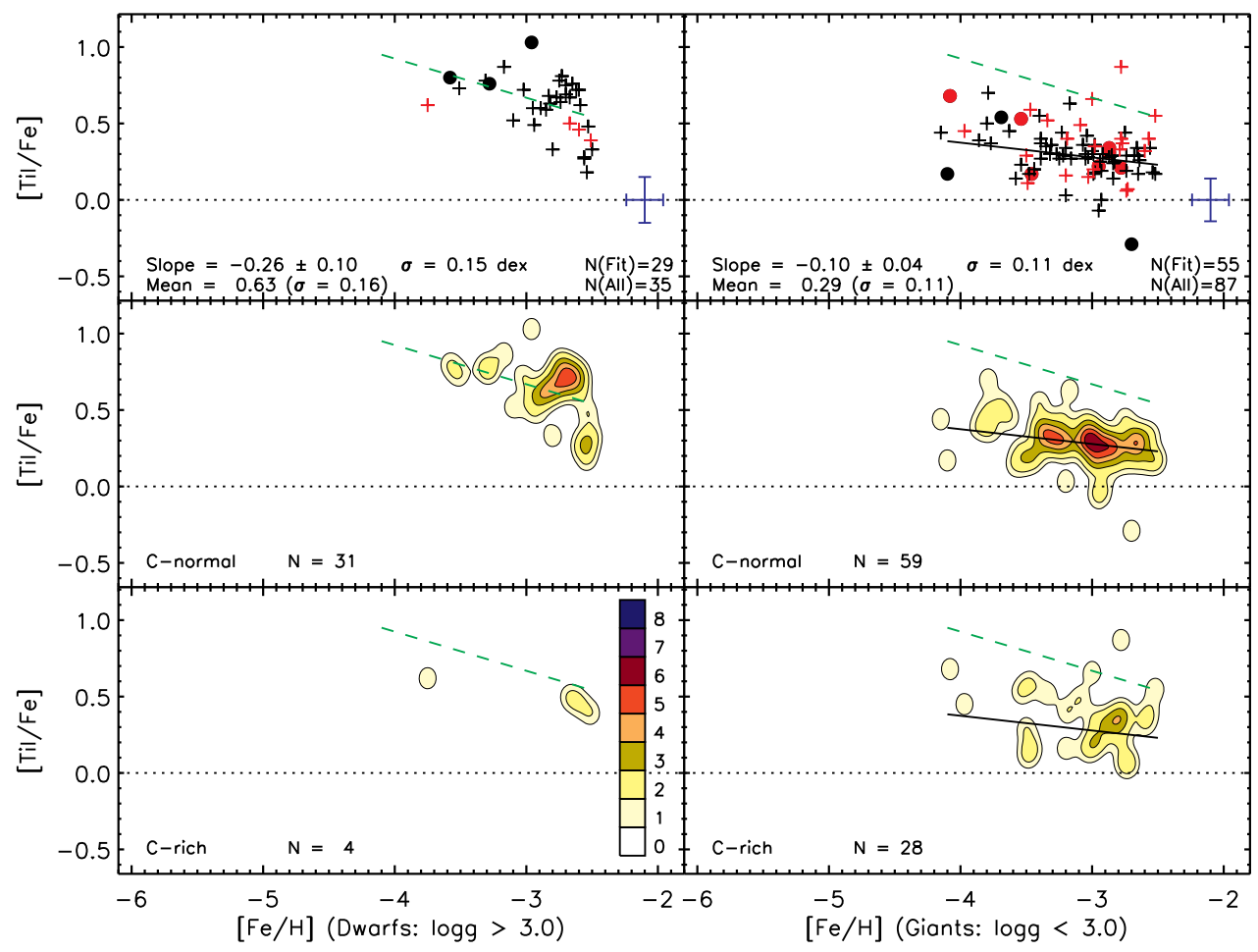

Figure 23. Same as Figure 17, but for [Ti I/Fe].

(A color version of this figure is available in the online journal.)

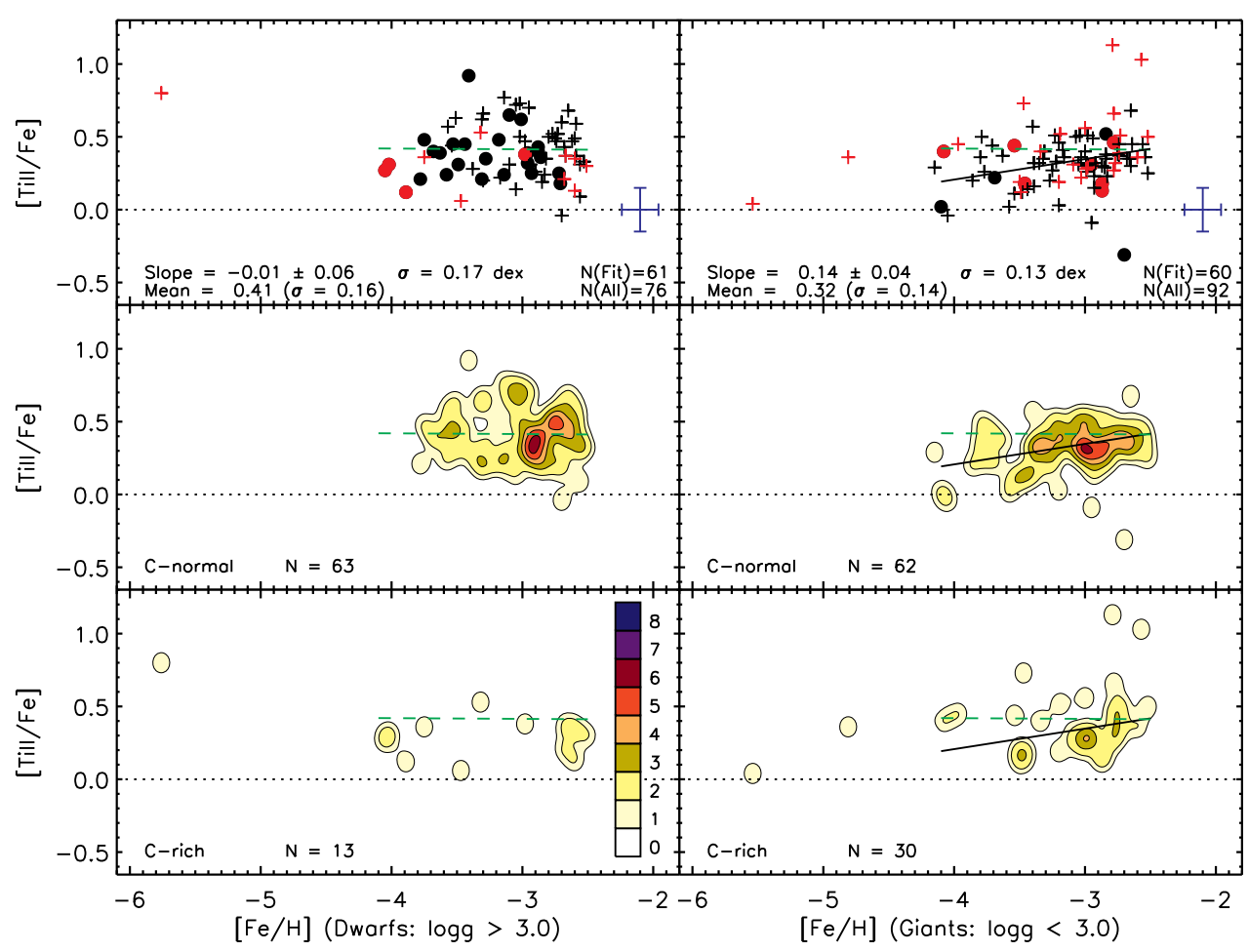

Figure 24. Same as Figure 17, but for [Ti II/Fe].

(A color version of this figure is available in the online journal.) 


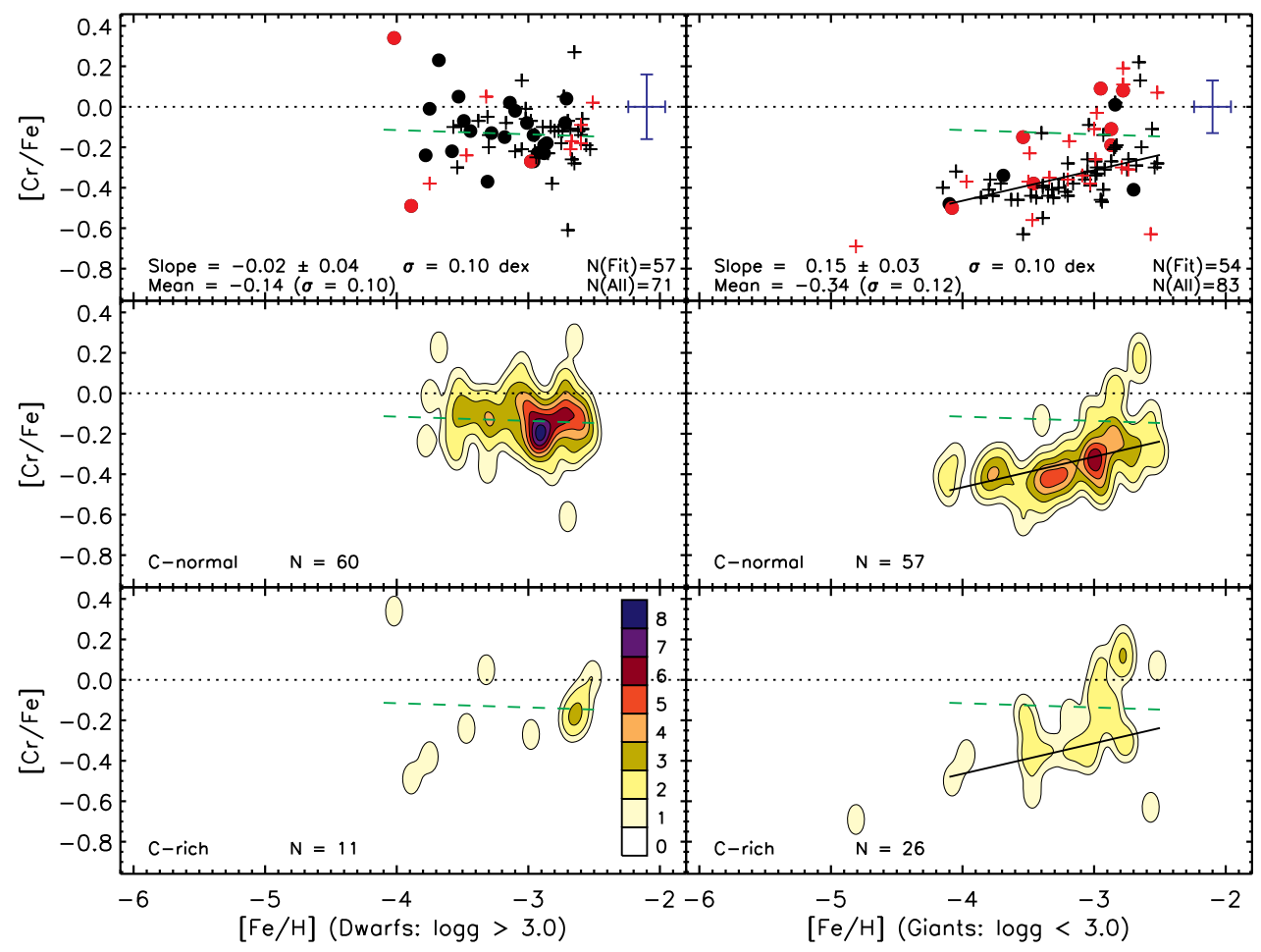

Figure 25. Same as Figure 17, but for $[\mathrm{Cr} / \mathrm{Fe}]$.

(A color version of this figure is available in the online journal.)

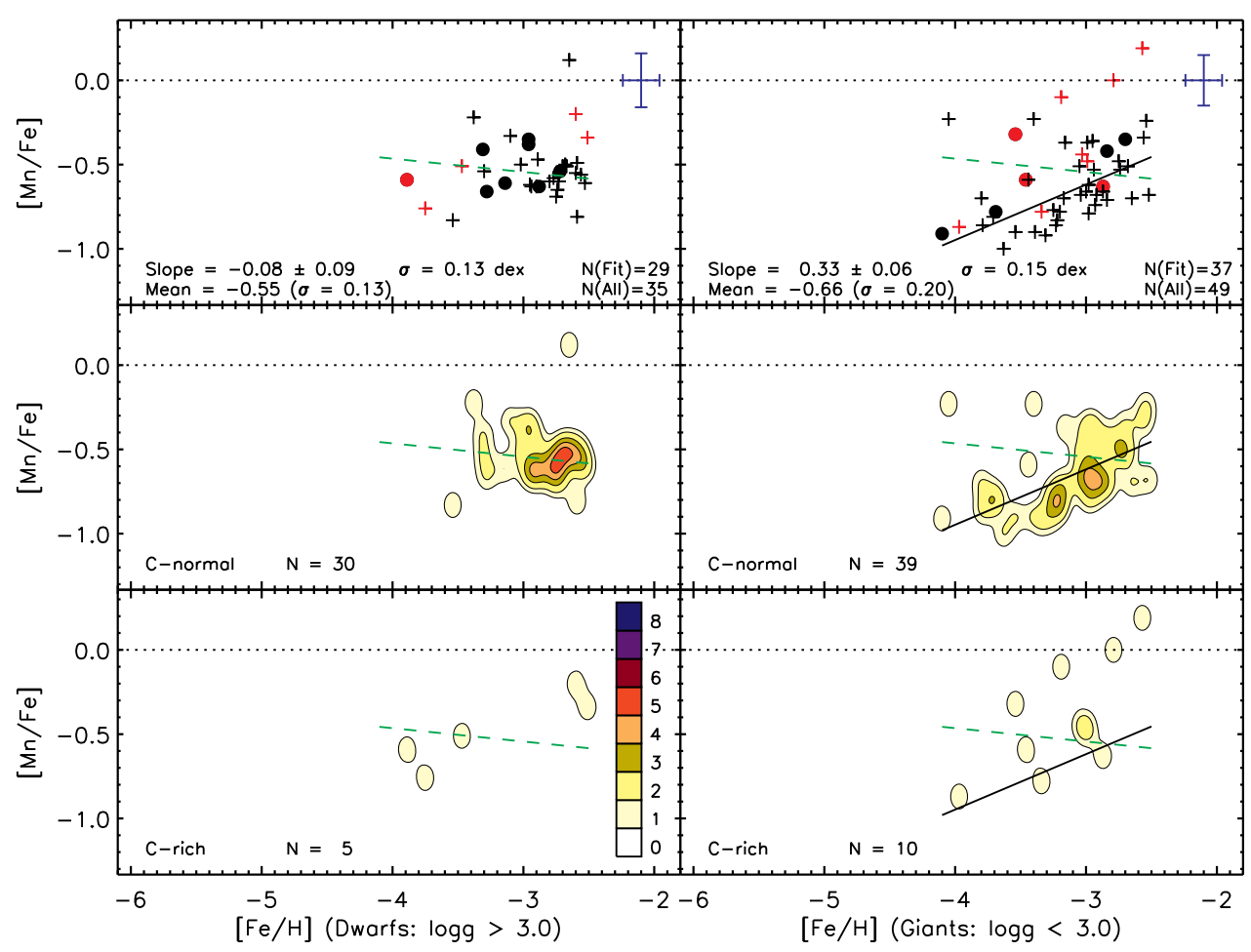

Figure 26. Same as Figure 17, but for $[\mathrm{Mn} / \mathrm{Fe}]$.

(A color version of this figure is available in the online journal.) 


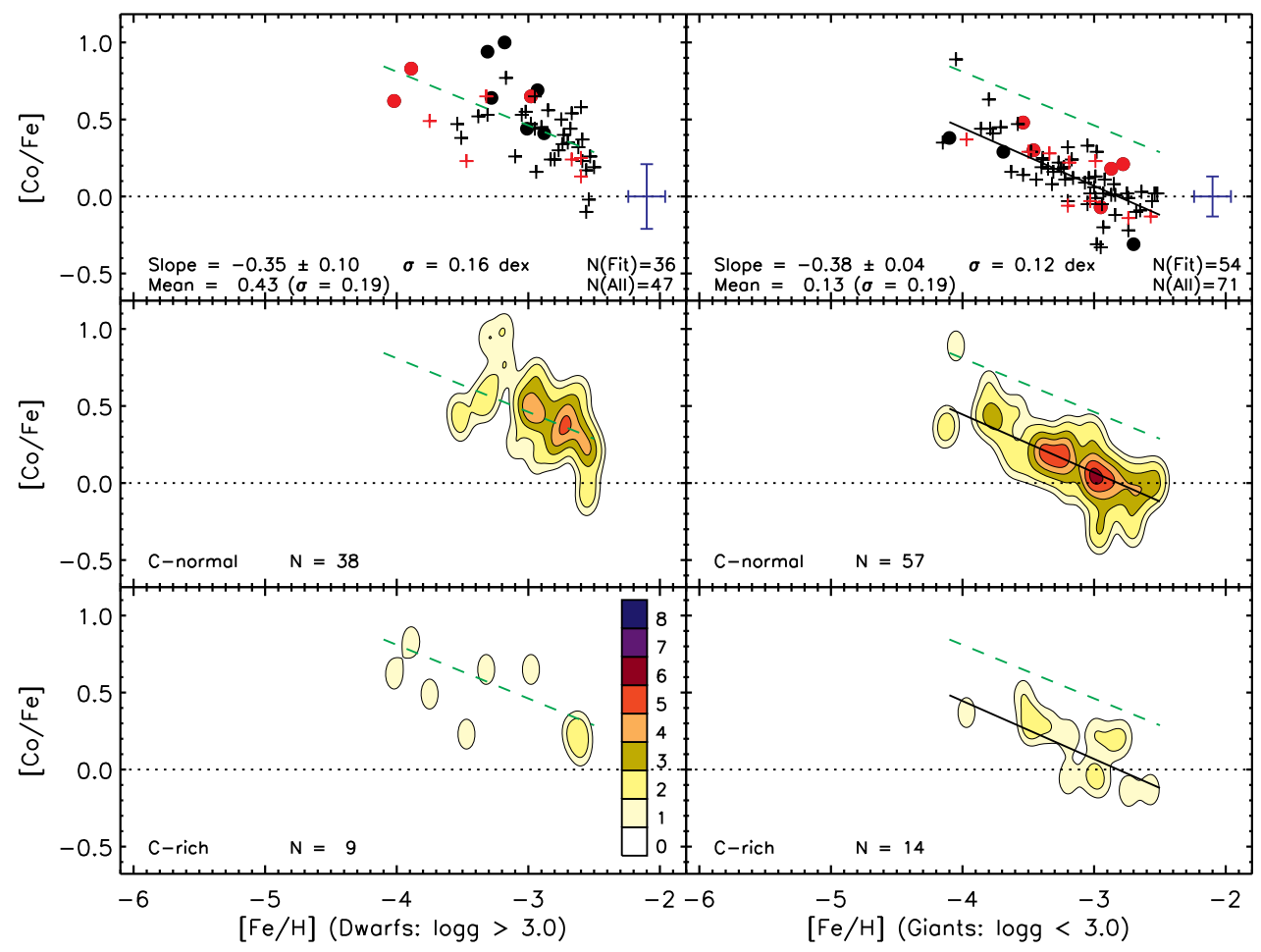

Figure 27. Same as Figure 17, but for $[\mathrm{Co} / \mathrm{Fe}]$.

(A color version of this figure is available in the online journal.)

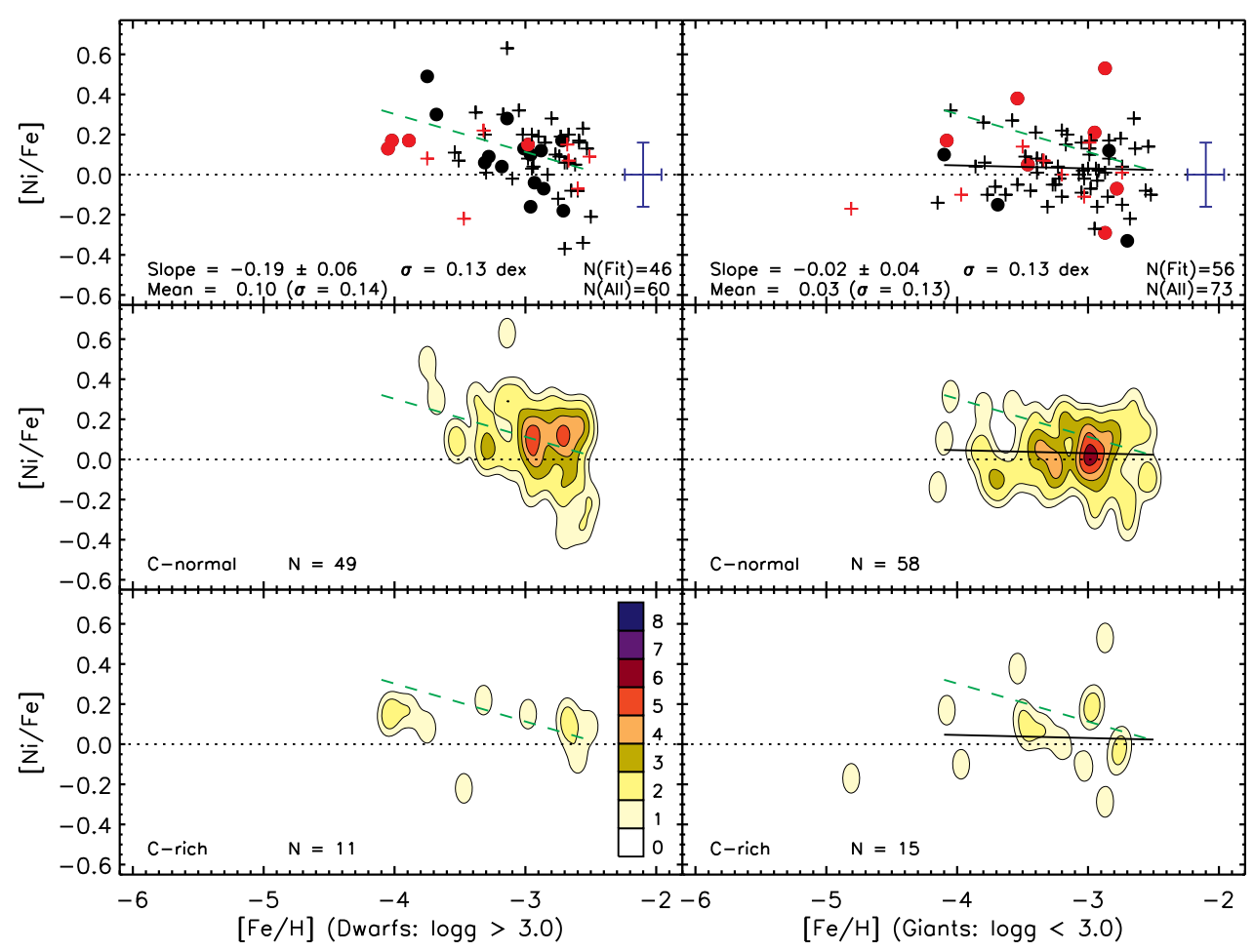

Figure 28. Same as Figure 17, but for $[\mathrm{Ni} / \mathrm{Fe}]$.

(A color version of this figure is available in the online journal.) 


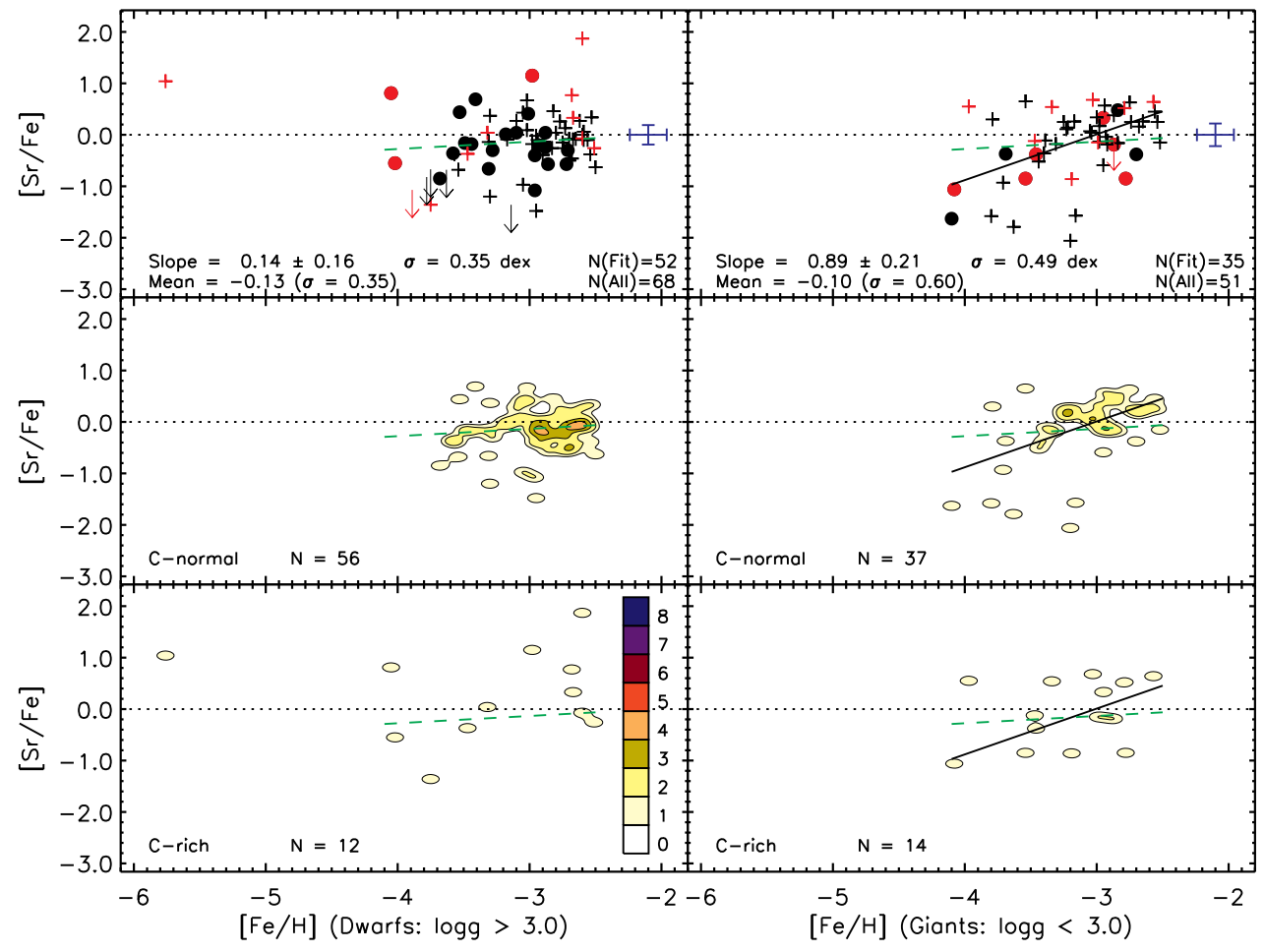

Figure 29. Same as Figure 17, but for $[\mathrm{Sr} / \mathrm{Fe}]$.

(A color version of this figure is available in the online journal.)

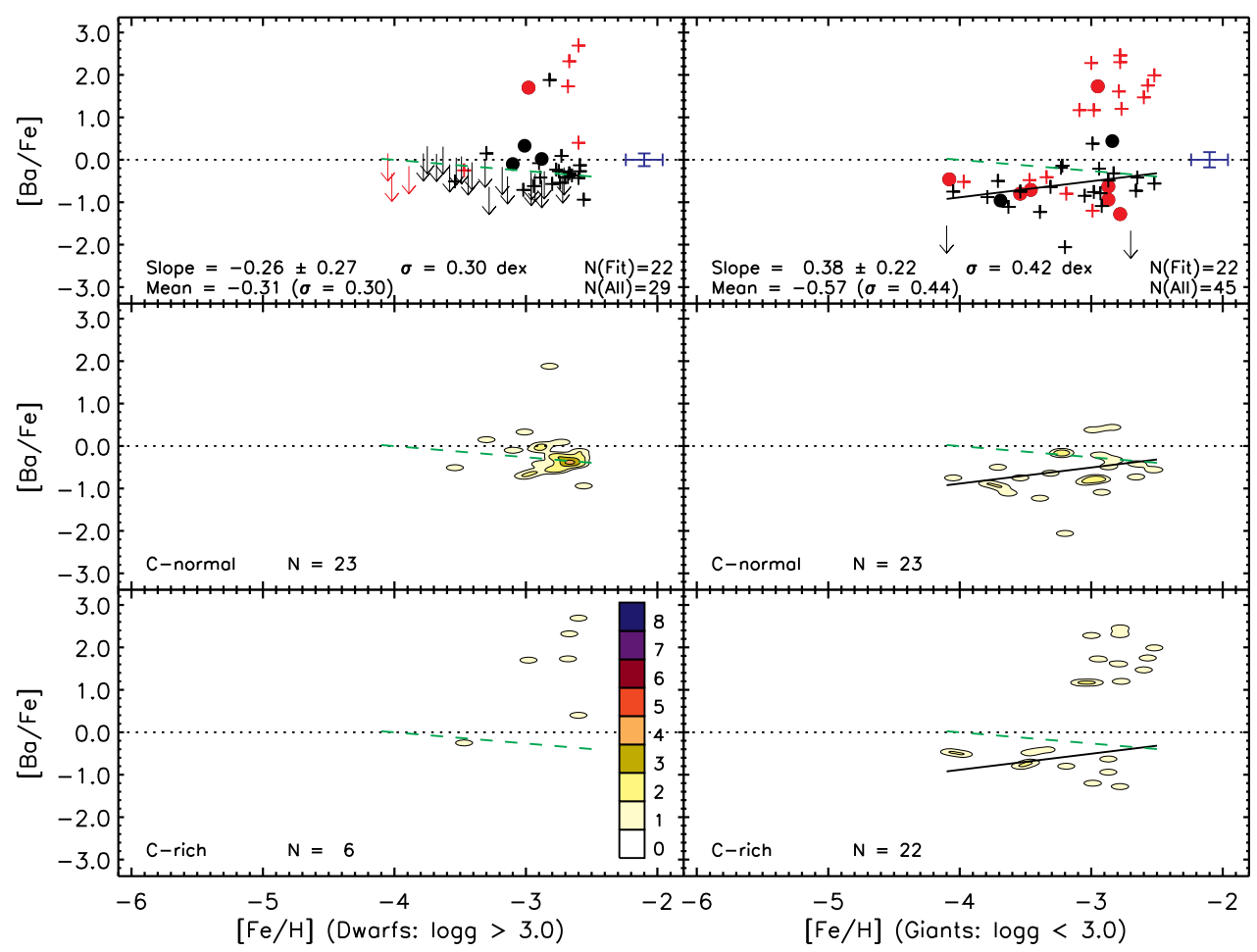

Figure 30. Same as Figure 17, but for $[\mathrm{Ba} / \mathrm{Fe}]$

(A color version of this figure is available in the online journal.) 


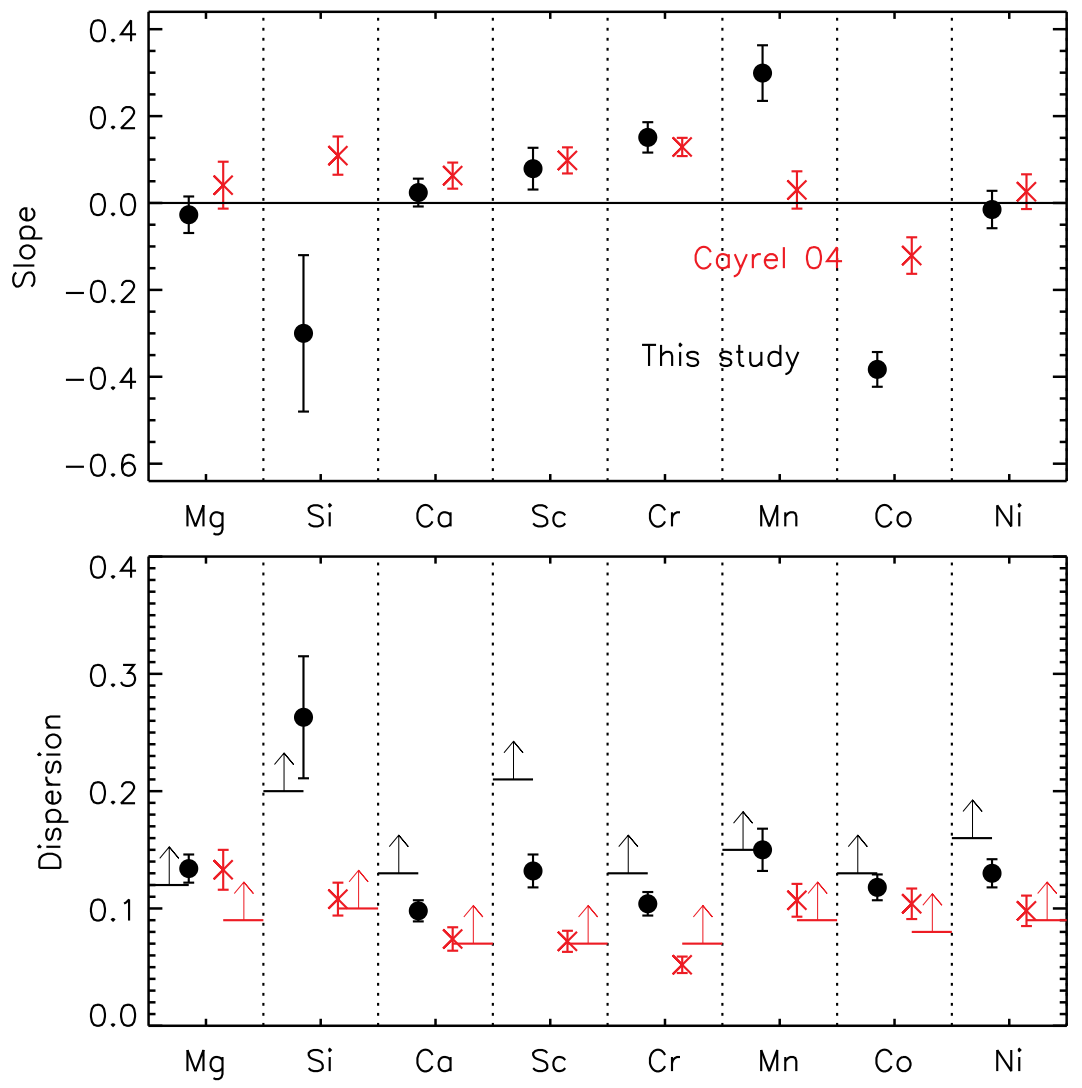

Figure 31. Comparison of the slopes of the linear fit to the data, $[\mathrm{X} / \mathrm{Fe}]$ vs. $[\mathrm{Fe} / \mathrm{H}]$, (upper) and the dispersion about the linear fit (lower) for our study (black circles) and the Cayrel et al. (2004) sample (red crosses). The linear fit excludes CEMP objects and $2 \sigma$ outliers. The horizontal bars (and upward facing arrows) in the lower panel indicate the representative measurement uncertainty.

(A color version of this figure is available in the online journal.)

maximum height of $N$. One advantage of using such plots is that the density of data points can be more readily seen, which may be useful given our sample size.

Although we determine linear fits to the data throughout this paper, we are not suggesting that this is the appropriate function to use. Rather, we consider this a first pass to begin to understand how the abundance ratios, $[\mathrm{X} / \mathrm{Fe}]$, evolve with metallicity, $[\mathrm{Fe} / \mathrm{H}]$. Such an approach has the advantage of enabling comparisons between different objects (dwarfs versus giants), different elements, and different studies. Without knowing what is the appropriate function to use, another option might be to follow Norris et al. (2001), and use LOESS regression lines (Cleveland 1979).

The reason for separating the dwarfs from giants is that we want to compare, as best we can, stars with similar stellar parameters to minimize systematic errors (e.g., Cayrel et al. 2004; Meléndez et al. 2008; Bonifacio et al. 2009; AlvesBrito et al. 2010; Nissen \& Schuster 2010). ${ }^{13}$ Additionally, we distinguish dwarfs from giants because, for some elements (notably $\mathrm{C}$ and $\mathrm{N}$ ), we anticipate abundance differences due to stellar evolution. Thus, by considering dwarfs and giants separately, we hope to minimize such effects and thereby more clearly identify a "normal" population of stars, and quantify the

\footnotetext{
13 We reiterate that our "dwarf" definition, $\log g>3.0$ will include subgiants as well as stars at the base of the giant branch. In reality, the two groups that we refer to as "giants" and "dwarfs" represent a "low-gravity" and a "high-gravity" group. Recall that the temperatures for the literature sample are internally consistent within each group.
}

Table 8

Comparison of Abundance Dispersions and Measurement Uncertainties

\begin{tabular}{|c|c|c|c|c|c|c|}
\hline \multirow[t]{2}{*}{ Species } & $\sigma^{\mathrm{a}}$ & $\begin{array}{l}\text { Measurement } \\
\text { Uncertainty }^{\mathrm{b}}\end{array}$ & $N_{\text {stars }}$ & $\sigma^{\mathrm{a}}$ & $\begin{array}{l}\text { Measurement } \\
\text { Uncertainty }^{\mathrm{b}}\end{array}$ & $N_{\text {stars }}$ \\
\hline & (2) & $\begin{array}{c}\text { Dwarfs } \\
\text { (3) }\end{array}$ & (4) & (5) & $\begin{array}{l}\text { Giants } \\
(6)\end{array}$ & (7) \\
\hline$[\mathrm{Na} / \mathrm{Fe}]$ & 0.14 & 0.18 & 18 & 0.21 & 0.18 & 38 \\
\hline$[\mathrm{Mg} / \mathrm{Fe}]$ & 0.17 & 0.12 & 58 & 0.13 & 0.12 & 60 \\
\hline$[\mathrm{Al} / \mathrm{Fe}]$ & 0.12 & 0.15 & 36 & 0.17 & 0.19 & 54 \\
\hline$[\mathrm{Si} / \mathrm{Fe}]$ & 0.18 & 0.20 & 48 & 0.26 & 0.20 & 14 \\
\hline$[\mathrm{Ca} / \mathrm{Fe}]$ & 0.15 & 0.13 & 62 & 0.10 & 0.13 & 60 \\
\hline$[\mathrm{Sc} / \mathrm{Fe}]$ & 0.13 & 0.20 & 53 & 0.13 & 0.21 & 44 \\
\hline [Ti I/Fe] & 0.15 & 0.15 & 29 & 0.10 & 0.14 & 55 \\
\hline [Ti II/Fe] & 0.17 & 0.15 & 61 & 0.13 & 0.15 & 60 \\
\hline$[\mathrm{Cr} / \mathrm{Fe}]$ & 0.10 & 0.16 & 57 & 0.10 & 0.13 & 54 \\
\hline$[\mathrm{Mn} / \mathrm{Fe}]$ & 0.13 & 0.16 & 29 & 0.15 & 0.15 & 37 \\
\hline$[\mathrm{Co} / \mathrm{Fe}]$ & 0.17 & 0.21 & 36 & 0.12 & 0.13 & 54 \\
\hline$[\mathrm{Ni} / \mathrm{Fe}]$ & 0.13 & 0.16 & 46 & 0.13 & 0.16 & 56 \\
\hline$[\mathrm{Sr} / \mathrm{Fe}]$ & 0.36 & 0.19 & 52 & 0.49 & 0.22 & 35 \\
\hline$[\mathrm{Ba} / \mathrm{Fe}]$ & 0.30 & 0.15 & 22 & 0.41 & 0.18 & 22 \\
\hline
\end{tabular}

Notes.

${ }^{a}$ Dispersions about the linear fit to the data in the $[\mathrm{X} / \mathrm{Fe}]$ vs. $[\mathrm{Fe} / \mathrm{H}]$ plane, for stars with $[\mathrm{Fe} / \mathrm{H}] \leqslant-2.5$, after discarding $2 \sigma$ outliers and CEMP objects, i.e., the values shown in Figures 17-30.

b Average measurement uncertainty for the program stars based on the data presented in Table 4. 


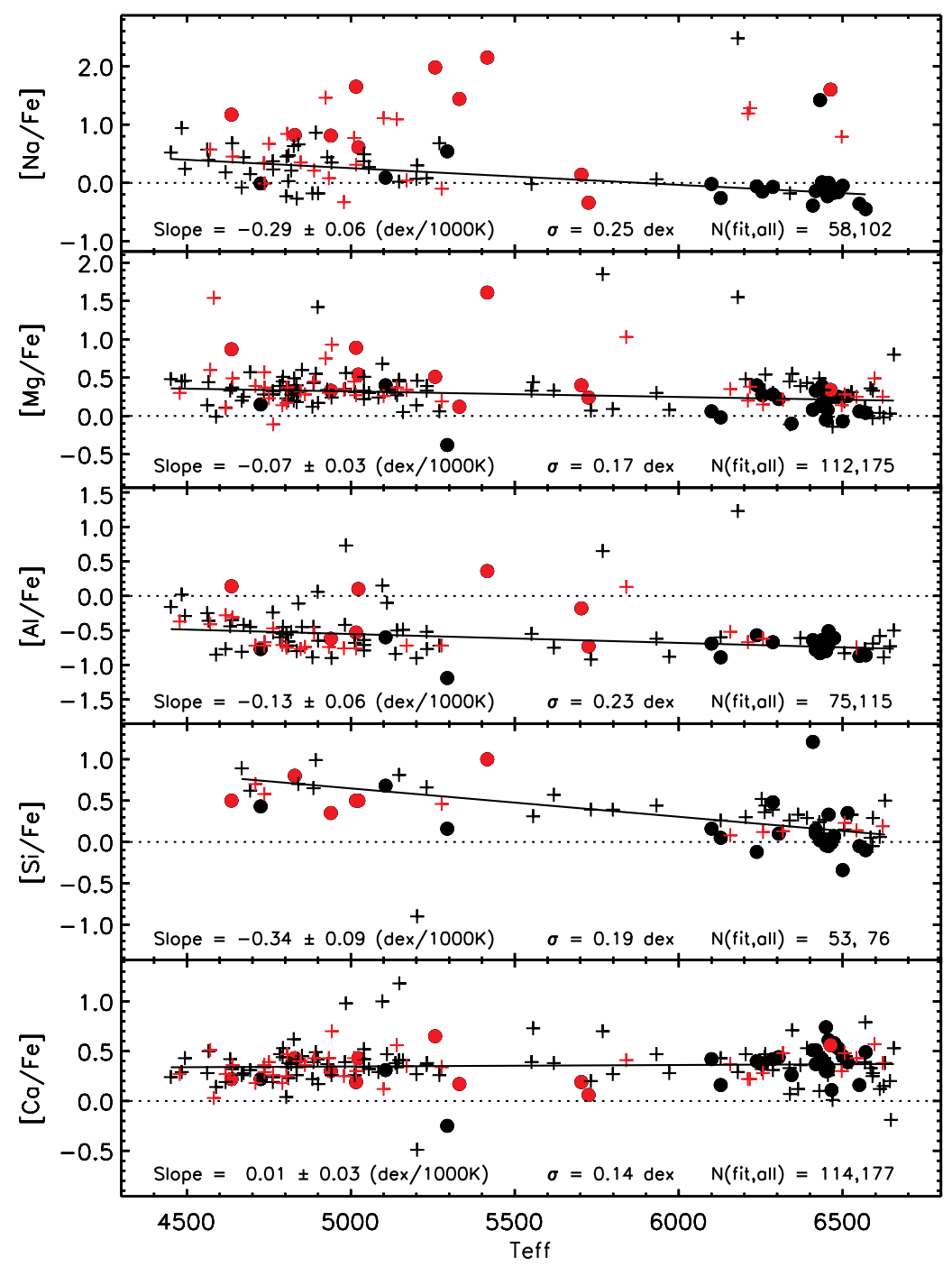

Figure 32. $[\mathrm{X} / \mathrm{Fe}]$ vs. $T_{\text {eff }}$ for $\mathrm{Na}, \mathrm{Mg}, \mathrm{Al}, \mathrm{Si}$, and $\mathrm{Ca}$. We only plot stars with $[\mathrm{Fe} / \mathrm{H}] \leqslant-2.5$. The symbols are the same as in Figure 15 . In each panel we show the linear fit to the data excluding CEMP objects and $2 \sigma$ outliers. In each panel we present the slope, uncertainty, dispersion about the linear fit, the number of stars involved in the fit, and the total number of stars plotted.

(A color version of this figure is available in the online journal.)

abundance trends $[\mathrm{X} / \mathrm{Fe}]$ versus $[\mathrm{Fe} / \mathrm{H}]$ for the "normal" dwarf sample and the "normal" giant sample.

A measure of (1) whether a "normal" population indeed exists, and (2) whether our selection criteria are able to identify such a population, is to compare the dispersion about the linear fit to the representative measurement uncertainty (the average "total error" for the program stars). (Such a comparison is only meaningful if, as we assume, the dependence of $[\mathrm{X} / \mathrm{Fe}]$ versus $[\mathrm{Fe} / \mathrm{H}]$ is linear. We reiterate that while we use linear functions, we are not suggesting that they are correct. Instead, this assumption represents a first step to understanding trends between $[\mathrm{X} / \mathrm{Fe}]$ and $[\mathrm{Fe} / \mathrm{H}]$.) In Table 8 , we compare the observed dispersion about the linear fit to the typical, i.e., average, measurement uncertainty (the lower panel of Figure 31 shows the comparison for a subset of elements in giant stars). In many cases (e.g., $\mathrm{Al}, \mathrm{Ca}, \mathrm{Ti}, \mathrm{Mn}, \mathrm{Co}, \mathrm{Ni}$ ), the dispersion in $[\mathrm{X} / \mathrm{Fe}]$ about the linear fit is in good agreement with the representative measurement uncertainty. There are examples in which the representative uncertainty exceeds the dispersion (e.g., Sc, Cr), which may indicate that the uncertainties are overestimated. For other cases (e.g., Sr, Ba), the dispersion about the linear trend far exceeds the measurement uncertainty, suggesting that the uncertainties are underestimated, there is a large abundance dispersion, and/or the "normal" population, if present, was not successfully identified.

\subsection{Dwarf versus Giant Differences}

Bonifacio et al. (2009) conducted a detailed analysis of metal-poor dwarf stars, and then compared their results with abundances from the Cayrel et al. (2004) metal-poor giants. For $\mathrm{Ca}, \mathrm{Ni}, \mathrm{Sr}$, and $\mathrm{Ba}$, they found very good agreement between the abundances from dwarfs and giants. However, for C, Sc, Ti, Cr, $\mathrm{Mn}$, and $\mathrm{Co}$, the abundances from dwarfs were roughly $0.2 \mathrm{dex}$ higher than those for giants; for $\mathrm{Mg}$ and $\mathrm{Si}$, the abundances from dwarfs were approximately 0.2 dex lower than for giants. Carbon was the only element in which the abundance difference between dwarfs and giants could be attributed to an astrophysical cause, namely mixing and nucleosynthesis in giants (Iben 1964). For some elements, the abundance discrepancies could be the result of neglecting non-LTE and/or 3D effects in the analysis. Bonifacio et al. (2009) advocated using the abundances from 


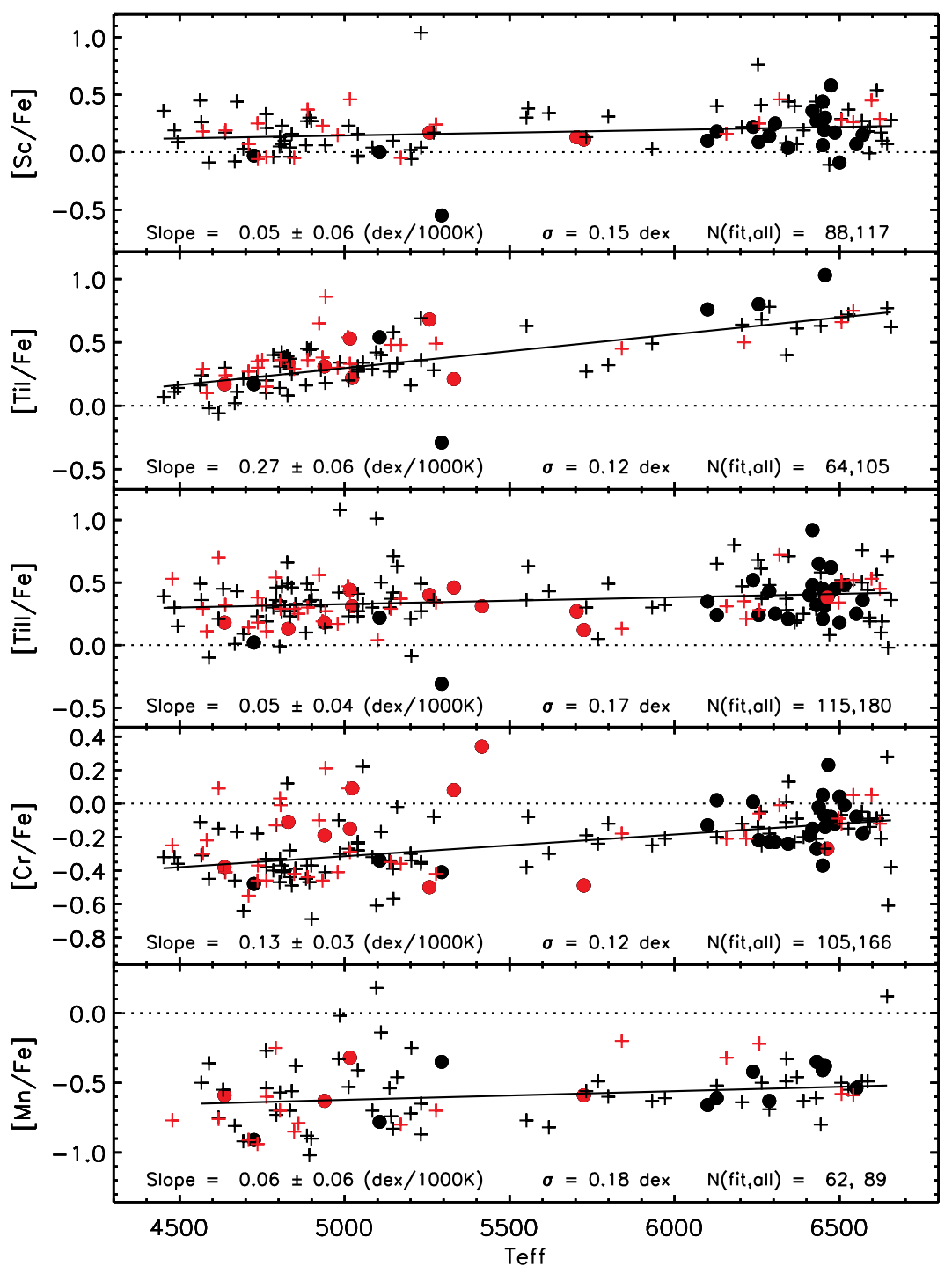

Figure 33. Same as Figure 32, but for Sc, Ti I, Ti II, Cr, and Mn.

(A color version of this figure is available in the online journal.)

giants for comparisons with chemical evolution models, because their 3D corrections were typically smaller than for dwarfs. On the other hand, Asplund (2005) found that non-LTE effects tend to be larger for giants. Also, Collet et al. (2011) argued that the CO ${ }^{5}$ BOLD 3D models used by Bonifacio et al. (2009) suffer from systematic errors in the high atmospheric layers, and thus underestimate the $3 \mathrm{D}$ effects.

For the complete sample, program stars + literature stars, we now use Figures 17-30 to compare abundances between giants and dwarfs. (For C and N, we defer to Spite et al. 2005 and Bonifacio et al. 2009, who have more accurate measurements for homogeneous samples of dwarfs and giants.) We consider the slope in $[\mathrm{X} / \mathrm{Fe}]$ versus $[\mathrm{Fe} / \mathrm{H}]$ and the mean abundance, for each element, having excluded CEMP stars and $2 \sigma$ outliers. For $\mathrm{Na}, \mathrm{Al}, \mathrm{Sc}, \mathrm{Ti}$ II, Cr, Mn, Ni, and $\mathrm{Sr}$, we note that the slope differs between dwarfs and giants at the $2 \sigma$ level or higher. The remaining elements, $\mathrm{Mg}, \mathrm{Si}, \mathrm{Ca}$, $\mathrm{Ti}$ I, $\mathrm{Co}$, and $\mathrm{Ba}$, exhibit slopes in $[\mathrm{X} / \mathrm{Fe}]$ versus $[\mathrm{Fe} / \mathrm{H}]$ that agree between dwarfs and giants. For $\mathrm{Na}, \mathrm{Si}$, Ti I, Cr, Co, and $\mathrm{Ba}$, the mean abundance between dwarfs and giants differs by more than 0.20 dex. For $\mathrm{Na}$ and $\mathrm{Si}$, the mean abundance for giants exceeds the mean abundance for dwarfs, while for Ti I, Cr, Co, and Ba, the mean abundance for dwarfs is higher than for giants. The sign of the differences for $\mathrm{Si}, \mathrm{Ti}$, and $\mathrm{Co}$ is the same as that found by Bonifacio et al. (2009). Consideration of the standard error of the mean would indicate that, for all elements except $\mathrm{Al}, \mathrm{Ca}, \mathrm{Ni}, \mathrm{Sr}$, and $\mathrm{Ba}$, the differences in the average abundances between the dwarf and giant samples are significant at the $3 \sigma$ level or higher. There is no obvious astrophysical cause for these abundance differences between dwarfs and giants; thus, we would attribute these abundance differences to non-LTE and/or 3D effects. We remind the reader that we have employed the same set of lines for giants and dwarfs, although the giants and dwarfs may use different subsets of lines for a given element.

Another way to view the abundance differences between dwarfs and giants is to plot the abundance ratios $[\mathrm{X} / \mathrm{Fe}]$ versus $T_{\text {eff }}$ (see Figures 32-34). Many elements exhibit clear trends, in particular, $\mathrm{Na}, \mathrm{Si}, \mathrm{Ti}, \mathrm{Cr}, \mathrm{Co}, \mathrm{Sr}$, and $\mathrm{Ba}$ are significant at the $3 \sigma$ level or higher. For $\mathrm{Na}, \mathrm{Si}$, and $\mathrm{Sr}$, the trend is negative (decreasing $[\mathrm{X} / \mathrm{Fe}]$ with increasing $T_{\text {eff }}$ ), while for the other elements, the trend is positive (increasing $[\mathrm{X} / \mathrm{Fe}]$ with increasing $T_{\text {eff }}$ ). Lai et al. (2008) found similar results for $\mathrm{Si}$, 


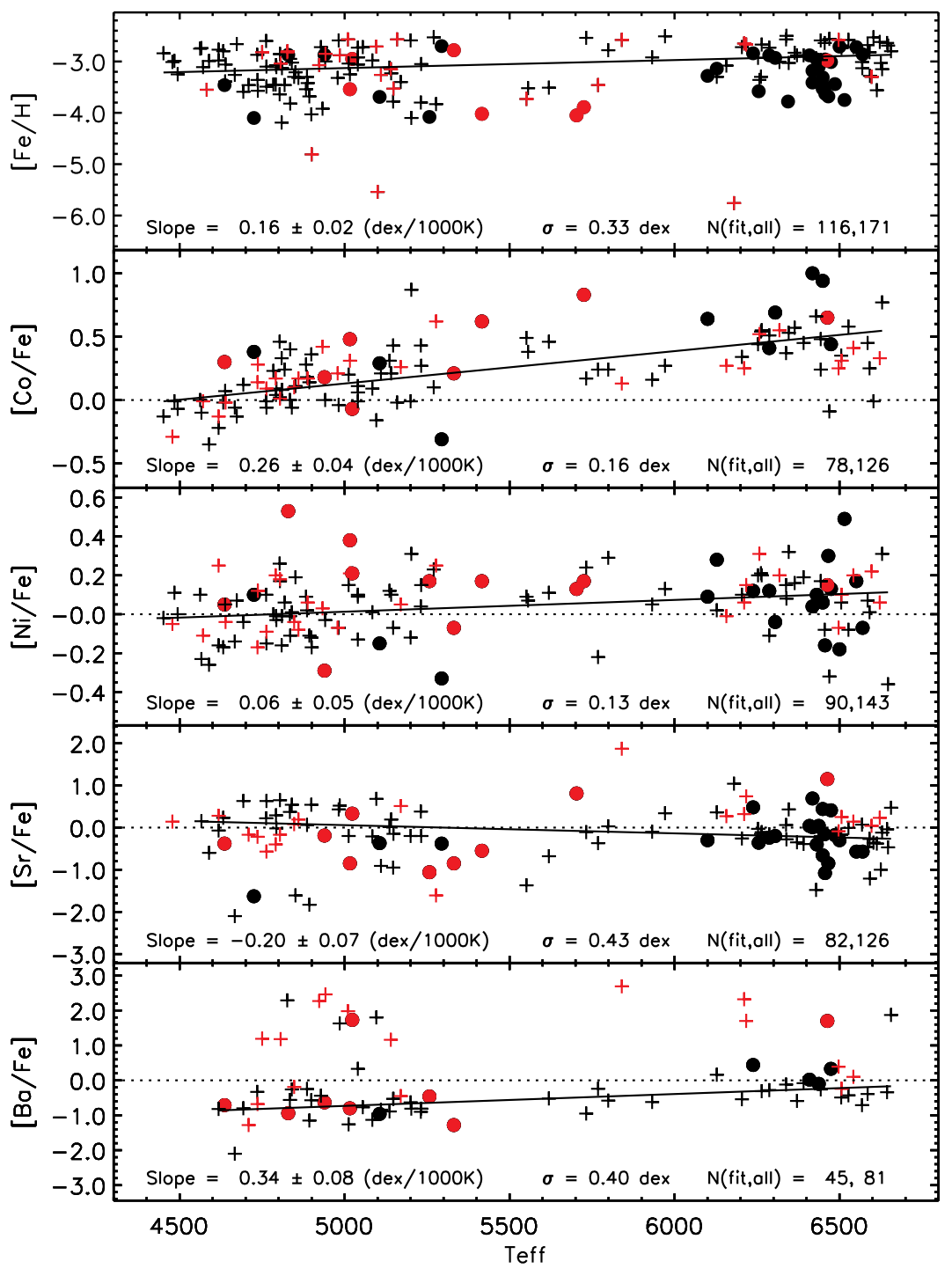

Figure 34. Same as Figure 32, but for $\mathrm{Fe}, \mathrm{Co}, \mathrm{Ni}, \mathrm{Sr}$, and $\mathrm{Ba}$.

(A color version of this figure is available in the online journal.)

$\mathrm{Ti}$, and $\mathrm{Cr}$, and speculated that they could be due to blends, deficiencies in the model atmospheres and/or inadequacies in spectral-line analysis techniques (e.g., non-LTE, 3D). They also caution that due care must be taken when comparing abundances of these elements with chemical evolution models or supernova yields. On looking at the data in Barklem et al. (2005), considering only stars with $[\mathrm{Fe} / \mathrm{H}] \leqslant-2.5$, we find that many elements (e.g., $\mathrm{Ca}, \mathrm{Co}, \mathrm{Cr}, \mathrm{Sc}$, and $\mathrm{Ti}$ ) exhibit a very significant trend ( $3 \sigma$ or higher) between $[\mathrm{X} / \mathrm{Fe}]$ versus $T_{\text {eff }}$. Therefore, we echo the Lai et al. (2008) concerns, and note the importance of restricting the range in $T_{\text {eff }}$ when possible (e.g., comparing dwarfs with dwarfs or giants with giants) in order to minimize systematic uncertainties, as done successfully by Arnone et al. (2005).

Finally, we note that radiative levitation and gravitational settling (sometimes called atomic diffusion) is suspected to play a role in altering the photospheric abundances between dwarf and giant stars at low metallicity (e.g., Richard et al. 2002a, $2002 \mathrm{~b}$ ). At present, the observational tests have been limited to the moderately metal-poor globular clusters NGC 6397 (Korn et al. 2007; Lind et al. 2008, 2009b) and NGC 6752 (Korn
2010). These analyses support the view that radiative levitation and gravitational settling can play an important role in producing abundance differences between dwarfs and giants. On looking at Figure 11 of Richard et al. (2002a), the model with $[\mathrm{Fe} / \mathrm{H}]=$ -3.31 predicts that $[\mathrm{Na} / \mathrm{Fe}]$ will be $\sim 0.3$ dex higher at $T_{\text {eff }}=$ $4500 \mathrm{~K}$ compared with $T_{\text {eff }}=6200 \mathrm{~K}$; this is in fair agreement with our observations. However, for $\mathrm{Si}$ and $\mathrm{Cr}$, the same models predict abundance differences between warmer dwarfs and cooler giants that are in the opposite sense to our findings. Ultimately, understanding the abundance differences between dwarfs and giants will require a combination of improved modeling in terms of non-LTE, 3D, and/or radiative levitation and gravitational settling.

\subsection{Non-LTE Na Abundances}

For Na, Lind et al. (2011) computed non-LTE abundance corrections for a number of lines, including the resonance lines used in our analysis. Their non-LTE corrections covered a large range in stellar parameters $\left(T_{\text {eff }}, \log g,[\mathrm{Fe} / \mathrm{H}]\right)$, as well as a large range in $[\mathrm{Na} / \mathrm{Fe}]$. In Figure 35, we apply the Lind et al. 

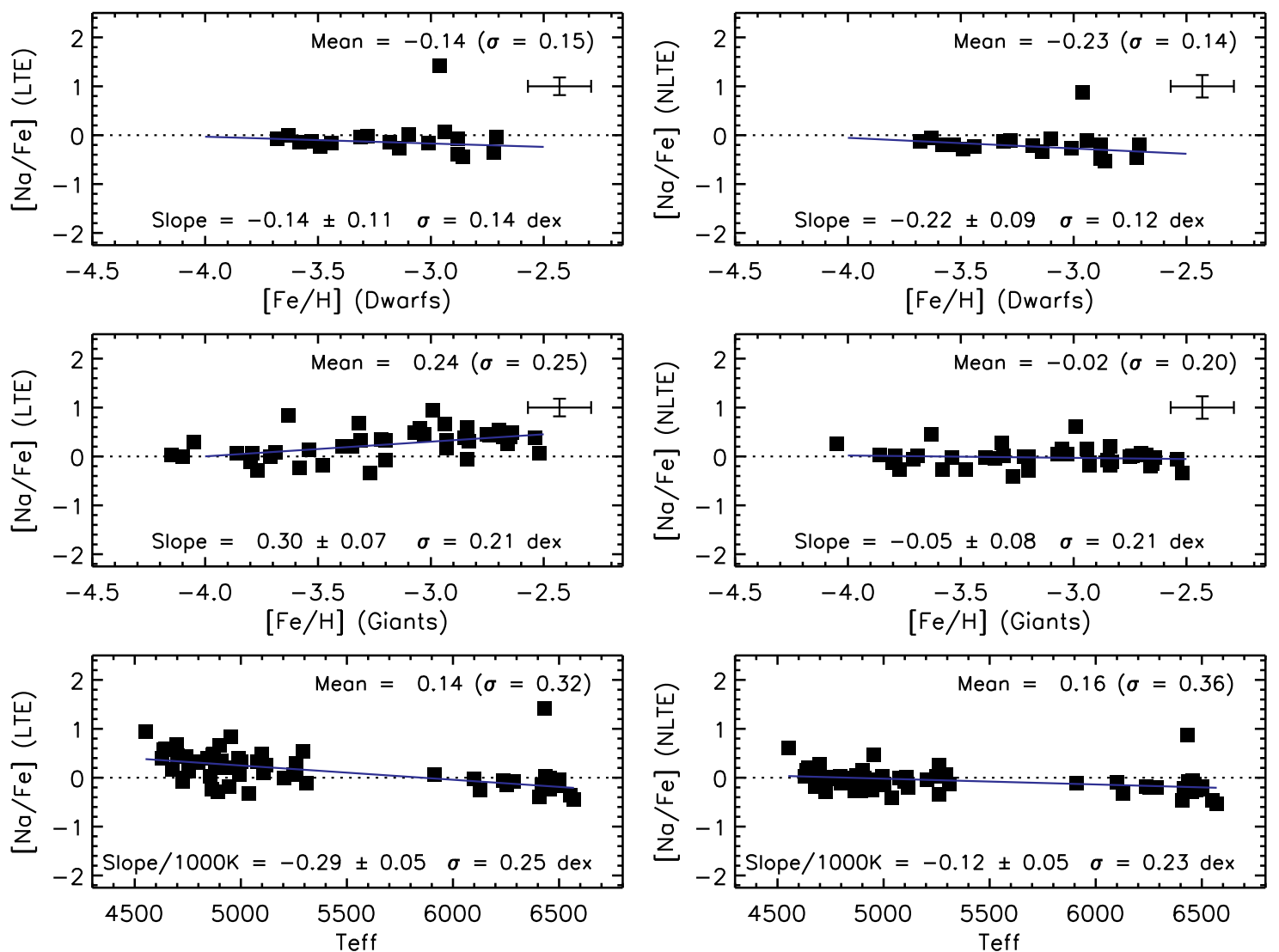

Figure 35. $[\mathrm{Na} / \mathrm{Fe}]$ vs. $[\mathrm{Fe} / \mathrm{H}]$ (upper and middle panels) and $[\mathrm{Na} / \mathrm{Fe}]$ vs. $T_{\text {eff }}$ (lower panels), excluding CEMP objects. The left and right panels correspond to LTE and non-LTE Na abundances, respectively, where the non-LTE corrections are taken from Lind et al. (2011). The upper panels present dwarf stars (log $g$ > 3.0) and the middle panels the giants $(\log g<3.0)$. In all panels we show the mean abundance and dispersion, the slope and uncertainty of the linear fit to the data (excluding $2 \sigma$ outliers), and the dispersion about the linear fit.

(A color version of this figure is available in the online journal.)

(2011) non-LTE corrections to the C-normal sample presented in Figure 17, for which $\mathrm{Na}$ abundances were all determined from the resonance lines. To reiterate, we exclude CEMP objects in this plot. To obtain the non-LTE corrections, we used linear interpolation for stars having stellar parameters $\left(T_{\text {eff }}, \log g\right.$, $[\mathrm{Fe} / \mathrm{H}]$ ) within the grid. For stars beyond the grid (those with $\log g<1.0$ or $[\mathrm{Fe} / \mathrm{H}] \leqslant-5.0$ ), we applied the nonLTE correction at the nearest boundary of the grid; thus, the corrections for those stars are uncertain. (Had we excluded the stars that lie beyond the grid, our results would be unchanged.)

In Figure 35, the left columns show LTE abundances. The top panels show dwarf stars and the middle panels show giants. In these panels, we again determine the linear fit to the data (excluding $2 \sigma$ outliers), and show the slopes and uncertainties, dispersions about the linear fit, and the mean abundance and dispersion. For the upper left and middle left panels, the numbers are the same as in Figure 17, as they should be. Furthermore, we note that $\mathrm{Na}$ is an element in which dwarfs and giants exhibit significant differences in their mean abundance, $[\mathrm{Na} / \mathrm{Fe}]$, and for the slope, $[\mathrm{Na} / \mathrm{Fe}]$ versus $[\mathrm{Fe} / \mathrm{H}]$.

In the right panels of Figure 35, we apply the Lind et al. (2011) non-LTE corrections, ${ }^{14}$ and determine the linear fit. (For clarity, we stress that only the $\mathrm{Na}$ abundances have been corrected, not the Fe abundances.) These corrections generally result in

\footnotetext{
14 We note that we applied the corrections for the $5895 \AA$ line to our average
} abundance and that the corrections for the 5889 A line are essentially identical. lower $\mathrm{Na}$ abundances, and therefore lower $[\mathrm{Na} / \mathrm{Fe}]$ ratios, than in the LTE case. For the giant sample, the slope changes from $0.30 \mathrm{dex} / \mathrm{dex}$ to $-0.05 \mathrm{dex} / \mathrm{dex}$, while for the dwarf sample, it changes from $-0.14 \mathrm{dex} / \mathrm{dex}$ to $-0.22 \mathrm{dex} / \mathrm{dex}$. Therefore, application of the non-LTE Na abundance corrections results in an improved agreement for the slope of $[\mathrm{Na} / \mathrm{Fe}]$ versus $[\mathrm{Fe} / \mathrm{H}]$ between dwarfs and giants. The slopes only differ at the $1.4 \sigma$ level, although the mean abundance difference is 0.21 dex. Similar results are obtained when using the Andrievsky et al. (2007) Na non-LTE corrections, although we note that their corrections assume an equivalent width corresponding to an LTE abundance of $[\mathrm{Na} / \mathrm{Fe}]=0$. The Lind et al. (2011) nonLTE corrections cover a large range in $[\mathrm{Na} / \mathrm{Fe}]$ at a given $T_{\text {eff }} / \log g /[\mathrm{Fe} / \mathrm{H}]$, and we expect (and find) the magnitude of the non-LTE correction to be a function of LTE Na abundance. For this sample, the non-LTE corrections are larger for giants than for dwarfs.

In the bottom panels of Figure 35 , we plot the $[\mathrm{Na} / \mathrm{Fe}]$ abundances (LTE and non-LTE) versus $T_{\text {eff }}$. Application of the Lind et al. (2011) Na non-LTE corrections results in a significantly shallower slope between $[\mathrm{Na} / \mathrm{Fe}]$ versus $T_{\text {eff }}$. This plot serves to highlight the importance of taking into account non-LTE effects, when possible, and as a useful exercise in assessing the importance of non-LTE corrections to the (1) mean abundance, (2) slope of $[\mathrm{X} / \mathrm{Fe}]$ versus $[\mathrm{Fe} / \mathrm{H}]$, and (3) trends between $[\mathrm{X} / \mathrm{Fe}]$ versus $T_{\text {eff }}$. We await with great interest more detailed grids of non-LTE abundance corrections 


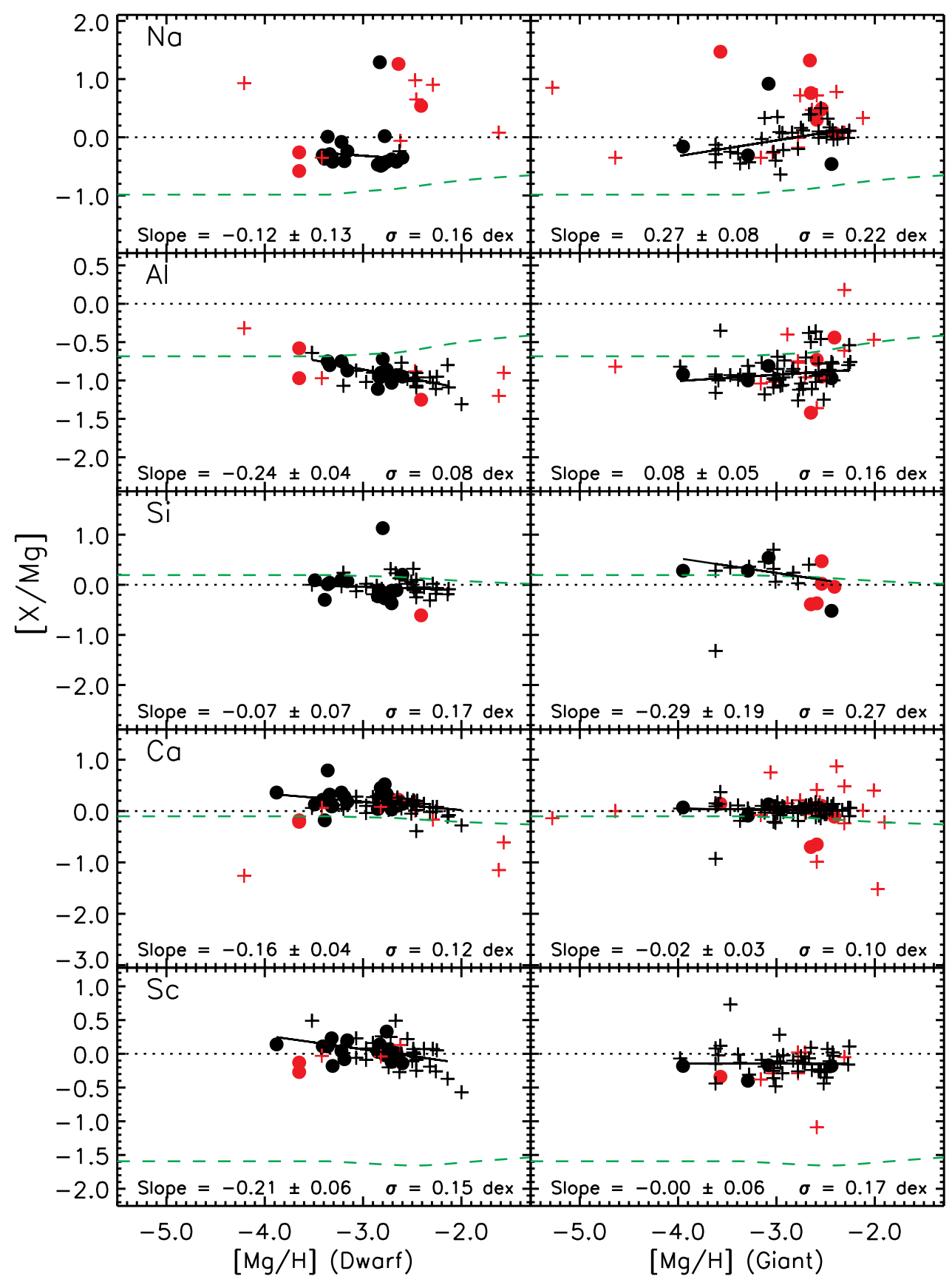

Figure 36. $[\mathrm{X} / \mathrm{Mg}]$ vs. $[\mathrm{Mg} / \mathrm{H}]$ for the elements $\mathrm{Na}$ to Sc. The left panels present dwarf stars $(\log g>3.0)$ and the right panels the giants $(\log g<3.0)$. Symbols are the same as in Figure 15. In each panel we show the linear fit to the data below $[\mathrm{Mg} / \mathrm{H}]=-2.0$, excluding CEMP objects and $2 \sigma$ outliers. The slope (and associated error) of this fit are presented as well as the dispersion about the best fit. The green dashed line represents the predictions from Kobayashi et al. (2006).

(A color version of this figure is available in the online journal.)

for additional elements as well as grids of 3D abundance corrections, although we recognize the magnitude of such efforts currently underway (e.g., Andrievsky et al. 2008, 2011; Collet et al. 2011; Bergemann \& Gehren 2008; Bergemann et al. 2010; Bergemann 2011; Bergemann et al. 2012; Dobrovolskas et al. 2012; Lind et al. 2009a, 2011, 2012; Spite et al. 2012).

\section{5. $\mathrm{Mg}$ as the Reference Element}

As discussed by Cayrel et al. (2004), one possibility to aid the interpretation of the abundances in metal-poor stars is to use $\mathrm{Mg}$ as the reference element rather than Fe. An advantage of such an approach is that the nucleosynthetic production of $\mathrm{Mg}$ during hydrostatic burning is well understood, whereas the synthesis of $\mathrm{Fe}$ is more complicated and not unique. On the other hand, a disadvantage of using $\mathrm{Mg}$ over $\mathrm{Fe}$ is that there are fewer lines from which the abundance is measured, thus the measurements are less accurate. Nevertheless, Cayrel et al. (2004) took this alternate approach and noted that, in the regime $[\mathrm{Mg} / \mathrm{H}] \leqslant$ -3.0 , there was a suggestion that all abundance ratios, $[\mathrm{X} / \mathrm{Mg}]$, were flat. The plateau value of $[\mathrm{X} / \mathrm{Mg}]$ at lowest $[\mathrm{Mg} / \mathrm{H}]$ may therefore reflect yields from the first generation of supernovae.

In Figures 36-38, we plot $[\mathrm{X} / \mathrm{Mg}]$ versus $[\mathrm{Mg} / \mathrm{H}]$ for dwarfs and giants; for comparison with the work of Cayrel 


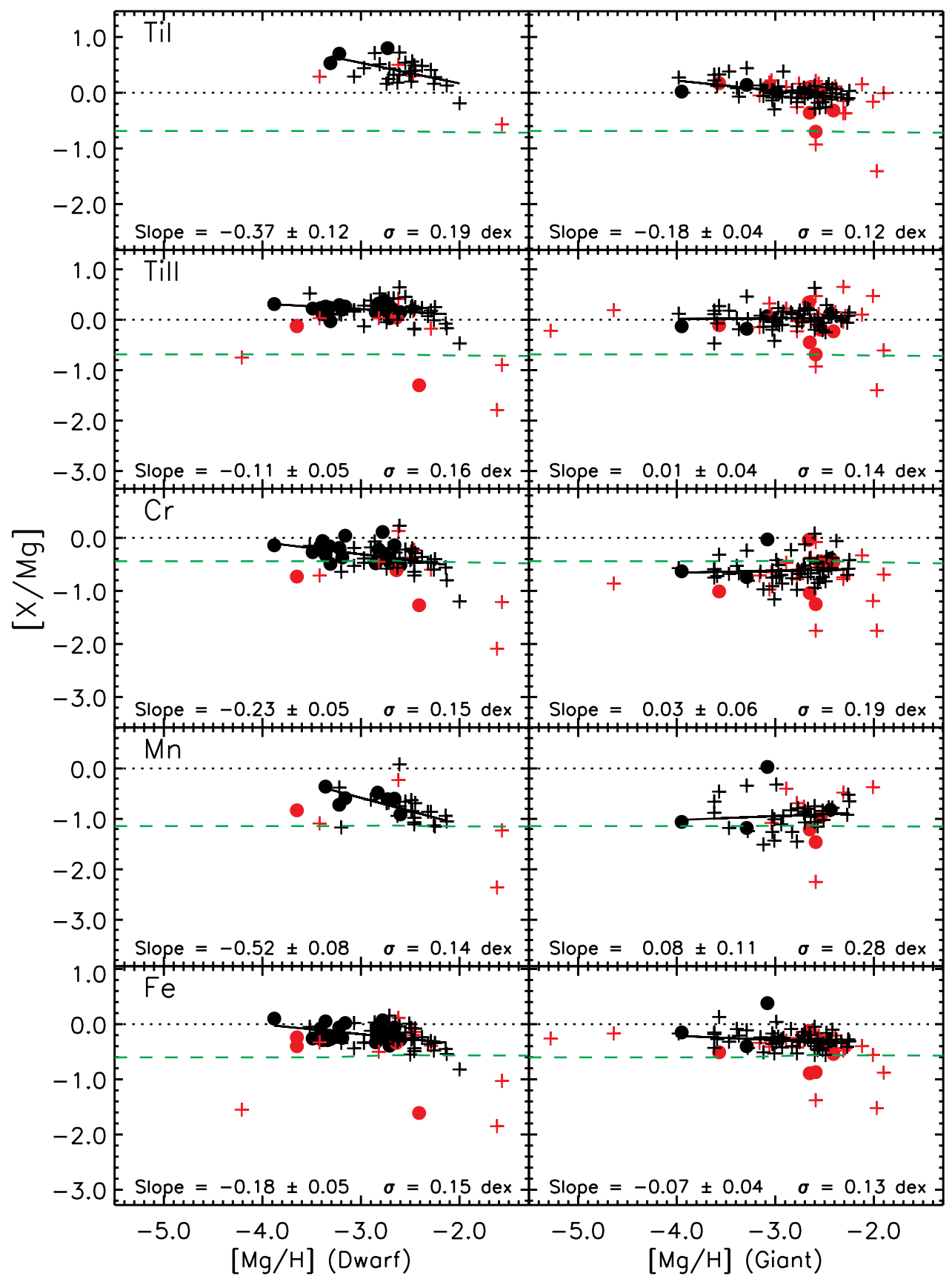

Figure 37. Same as Figure 36, but for the elements Ti I to Fe.

(A color version of this figure is available in the online journal.)

et al. (2004), in the range $[\mathrm{Mg} / \mathrm{Fe}] \leqslant-3.0$, we fit the data excluding CEMP stars and $2 \sigma$ outliers. In these figures we again include the predictions from Kobayashi et al. (2006). We reach similar conclusions to those of Cayrel et al. (2004). Namely, the dispersion about the linear fit is generally greater when plotting $[\mathrm{X} / \mathrm{Mg}]$ versus $[\mathrm{Mg} / \mathrm{H}]$ than for $[\mathrm{X} / \mathrm{Fe}]$ versus $[\mathrm{Fe} / \mathrm{H}]$ (although there are a few cases in which the opposite is true, e.g., $\mathrm{Al}, \mathrm{Si}$, and $\mathrm{Ti} \mathrm{II})$. This is presumably due to the $\mathrm{Mg}$ measurements being more uncertain than $\mathrm{Fe}$ due to the smaller number of lines. (Note that we are only fitting stars with $[\mathrm{Mg} / \mathrm{H}] \leqslant-3.0$ rather than the full abundance range.) For most elements, the linear fit to the data with $[\mathrm{Mg} / \mathrm{H}] \leqslant-3.0$ shows zero slope (at the $2 \sigma$ level), with notable exceptions including $\mathrm{Al}$ (dwarfs), Ti I (giants), and Co (dwarfs and giants). Considering stars in the range $[\mathrm{Mg} / \mathrm{H}] \leqslant-2.0$, rather than $[\mathrm{Mg} / \mathrm{H}] \leqslant-3.0$, we note that the linear fit to the data is not consistent with zero slope for a larger number of elements. In general, the behavior of $[\mathrm{X} / \mathrm{Mg}]$ versus $[\mathrm{Mg} / \mathrm{H}]$ exhibits a similar behavior at all metallicities. It is difficult to assess whether the Kobayashi et al. (2006) predictions are a better match to the $[\mathrm{X} / \mathrm{Mg}]$ or the $[\mathrm{X} / \mathrm{Fe}]$ plots. Ultimately, using $\mathrm{Mg}$ as the reference element does not seem to offer any major advantages over Fe, at least in this analysis.

Furthermore, we note that Chieffi et al. (2000) have suggested that $\mathrm{Si}, \mathrm{S}, \mathrm{Ar}$, and $\mathrm{Ca}$ (or combinations of these elements) may be better reference elements and tracers of Galactic chemical evolution than $\mathrm{Fe}$ (or O). Chieffi et al. (2000) argue that the yields of these elements are not strongly dependent on the 


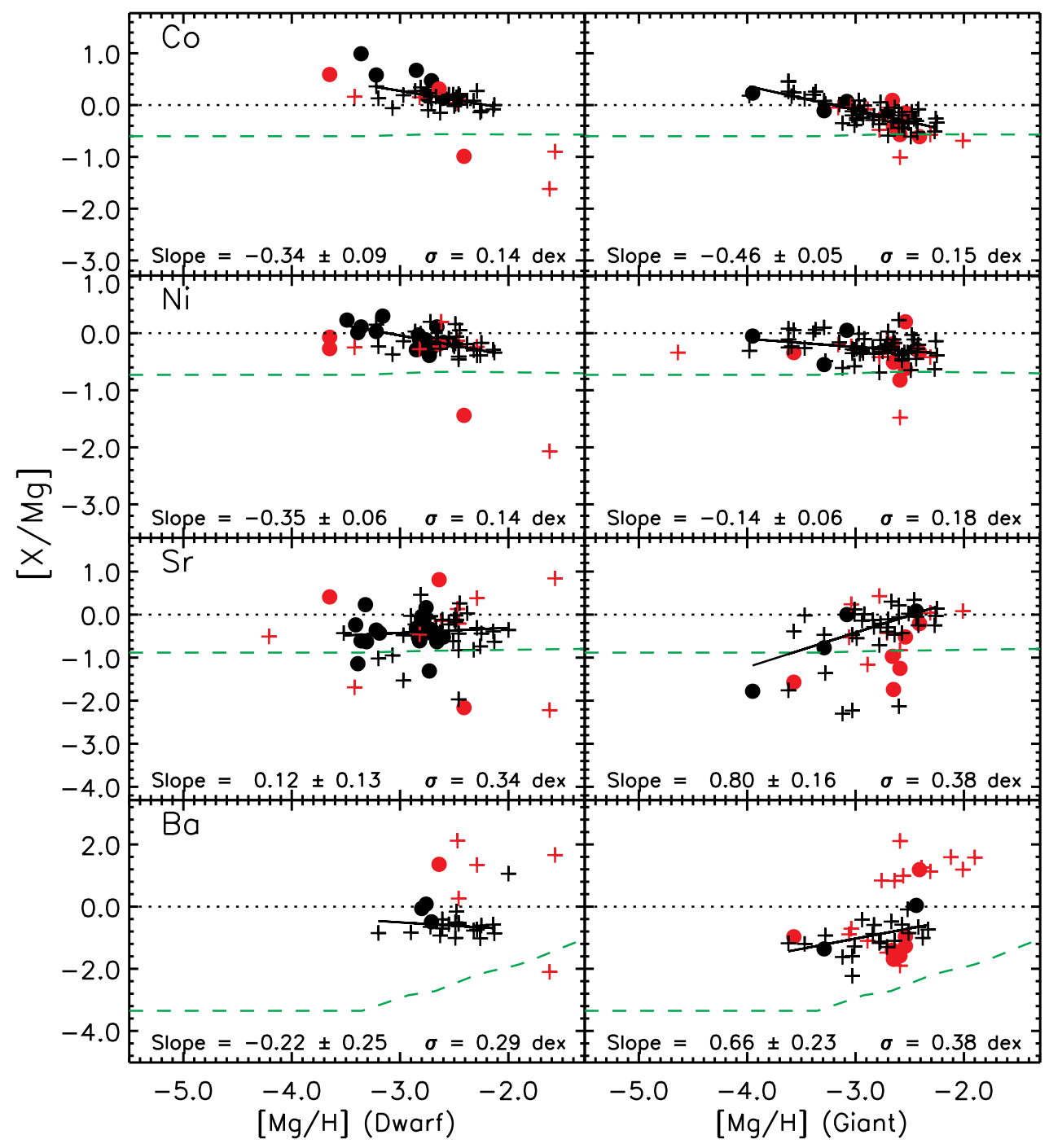

Figure 38. Same as Figure 36, but for the elements Co to Ba.

(A color version of this figure is available in the online journal.)

location of the mass cuts employed in the supernova explosion calculation or with the pre-explosive chemical composition. Of these elements, Ca has the most number of lines measured in our program stars, although the average number of lines is smaller than for $\mathrm{Mg}$, and the average total error for $\mathrm{Ca}$ exceeds that of $\mathrm{Mg}$.

\subsection{Additional Comparisons with Cayrel et al. (2004)}

In the upper panel of Figure 31, we compare the slope from the linear fit of $[\mathrm{X} / \mathrm{Fe}]$ versus $[\mathrm{Fe} / \mathrm{H}]$ between our sample (program + literature stars) and the Cayrel et al. (2004) study. That is, we are comparing the coefficient $a$ from the relation, $[\mathrm{X} / \mathrm{Fe}]=a \times[\mathrm{Fe} / \mathrm{H}]+b$, describing the best fit to our data and the best fit to their data. Since their sample consists exclusively of giants, we use the slope as determined from our giant sample in the comparison. To ensure that this was a proper comparison of gradients, we used our software to determine the slope (and uncertainty) to the Cayrel et al. (2004) data, rather than relying upon the values in their Table 9. As with our sample, we exclude the two CEMP stars and $2 \sigma$ outliers when determining the linear fit to the Cayrel et al. (2004) data. Figure 31 shows that, for Mg,
$\mathrm{Ca}, \mathrm{Sc}, \mathrm{Cr}$, and $\mathrm{Ni}$, the gradients measured in this study and Cayrel et al. (2004) are in very good agreement. For Si, Mn, and $\mathrm{Co}$, the slopes differ by more than $2 \sigma$. For $\mathrm{Si}$, the sign of the gradient differs between the two studies.

In the lower panel of this figure, we also compare the scatter about the linear fit between our giant sample and that of Cayrel et al. (2004). For their data, we again used our software to determine the scatter about the linear fit after eliminating $2 \sigma$ outliers and the two CEMP stars. (We used the relation given in Taylor (1997) for the fractional uncertainty in the dispersion, $1 / \sqrt{2(N-1)}$.) Some elements $(\mathrm{Mg}, \mathrm{Ca}, \mathrm{Mn}, \mathrm{Co}$, and $\mathrm{Ni}$ ) exhibit very good agreement. The elements that differ by more than $2 \sigma$ are $\mathrm{Si}, \mathrm{Sc}$, and $\mathrm{Cr}$. It is not surprising that, for all elements, the dispersions about the linear fit to the Cayrel et al. (2004) data are always equal to or smaller than for our sample of program and literature stars. This could be due, in part, to the fact that the Cayrel et al. (2004) sample is very homogeneous, and their spectra are of very high quality. While our sample includes their stars, we have a more heterogeneous sample, albeit one that was analyzed in a homogeneous manner.

In the lower panel of Figure 31, we also plot a representative measurement uncertainty for each element, the average "total 

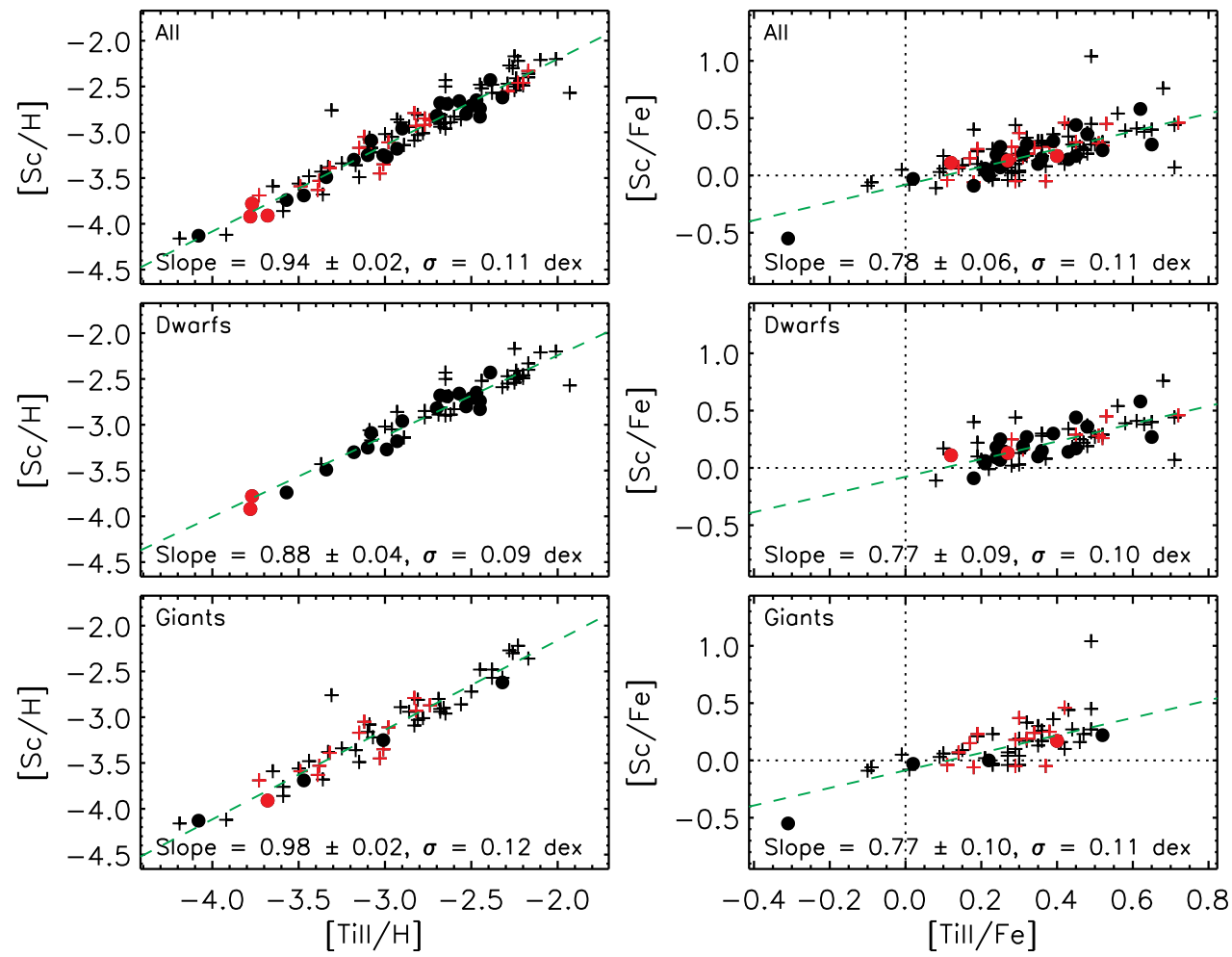

Figure 39. $[\mathrm{Sc} / \mathrm{H}]$ vs. [Ti II/H] (left panels) and [Sc/Fe] vs. [Ti II/Fe] (right panels) for all stars (upper panels), dwarf stars (middle panels), and giant stars (bottom panels). The symbols are the same as in Figure 15, and in each panel we present the linear fit to the data, excluding CEMP objects and $2 \sigma$ outliers. The slope, uncertainty, and dispersion about the slope are shown.

(A color version of this figure is available in the online journal.)

error" for the program stars. For the Cayrel et al. (2004) data, we also plot their estimate of the expected scatter for each element, based on their measurement uncertainties. These are shown as upward facing arrows, and we would expect the observed dispersions to lie on or above these values (for species having an astrophysically significant dispersion). For the Cayrel et al. (2004) data, the observed dispersions are in good agreement with the expected scatter. For our data, some elements $(\mathrm{Mg}$, $\mathrm{Mn}, \mathrm{Co}$ ) exhibit an observed dispersion in good agreement with the expected scatter. However, for Si, the observed dispersion exceeds the expected value; for Sc, the observed dispersion is considerably smaller than the expected value, which may indicate that our measurement uncertainties are overestimated for this element. Nevertheless, it is reassuring that in several cases $(\mathrm{Mg}, \mathrm{Ca}, \mathrm{Co}$, and $\mathrm{Ni}$ ) our dispersions about the linear fit are comparable with the values of Cayrel et al. (2004), suggesting that if indeed a "normal" population exists, our selection criteria were effective in identifying this population, even though our sample of program and literature stars is quite heterogeneous.

\subsection{Comparison with Predictions, and the Curious Case of Scandium and Titanium}

In Figure 14 and Figures 36-38, we overplot the Kobayashi et al. (2006) predictions of Galactic chemical enrichment. Their chemical evolution model includes the following assumptions: (1) one zone centered on the solar neighborhood, (2) no instantaneous recycling approximation, (3) contributions from hypernovae with large explosion energy $\left(E_{51}>10\right)$, Type II supernovae, and Type Ia supernovae, (4) no contributions from low- and intermediate-mass stars, and (5) infall of primordial gas. Figure 14 shows the evolution of $[\mathrm{X} / \mathrm{Fe}]$ against $[\mathrm{Fe} / \mathrm{H}]$. In general, the predictions provide a fair fit to the data in terms of the slope (or lack thereof). For many elements, the mean predictions differ from the mean observations by $\sim 0.5$ dex or more.

As reported in other investigations (e.g., Kobayashi et al. 2006), the predictions for Ti and Sc are underabundant relative to the LTE measurements. In our study, Sc measurements are exclusively from $\mathrm{Sc}$ II lines. We find that the abundance ratios $[\mathrm{Ti} \mathrm{II} / \mathrm{Fe}]$ and $[\mathrm{Sc} / \mathrm{Fe}]$ (and $[\mathrm{Ti} \mathrm{II} / \mathrm{H}]$ versus $[\mathrm{Sc} / \mathrm{H}]$ ) exhibit a highly significant, $\sim 10 \sigma$, correlation (see Figure 39 ). In this figure, we show all stars (upper panel), dwarfs (middle panel), and giants (lower panel). For each sample, the scatter about the mean trend is only $\sim 0.10 \mathrm{dex}$, which is substantially lower than the average total error for either [Ti II $/ \mathrm{Fe}](0.14 \mathrm{dex})$ or $[\mathrm{Sc} / \mathrm{Fe}]$ $(0.20$ dex $)$. We also find a correlation between $[\mathrm{Ti} \mathrm{I} / \mathrm{Fe}]$ and $[\mathrm{Sc} / \mathrm{Fe}]$, but with a lower significance $(\sim 2 \sigma)$ and shallower slope $(0.19 \mathrm{dex} / \mathrm{dex}$ to $0.38 \mathrm{dex} / \mathrm{dex})$. Although $\mathrm{Sc}$ and $\mathrm{Ti}$ are produced via different processes, the correlation we find suggests that the two elements might be produced in similar conditions. Umeda \& Nomoto (2005) suggest that the yield of $\mathrm{Sc}$ in metal-poor supernovae can be greatly increased in lowdensity (i.e., high-entropy) regions. Kobayashi et al. (2011a) suggest that the $v$-process in core-collapse supernovae may produce Sc, although to our knowledge there have been few, if any, studies of the yields of Sc from the $v$-process. The strong correlation between $\mathrm{Sc}$ and $\mathrm{Ti}$ found here might suggest that the $v$-process does not provide a complete explanation of the production of Sc at lowest metallicities.

Figures 36-38 show the evolution of $[\mathrm{X} / \mathrm{Mg}]$ against $[\mathrm{Mg} / \mathrm{H}]$. Again, the predictions provide a fair fit to the slope 


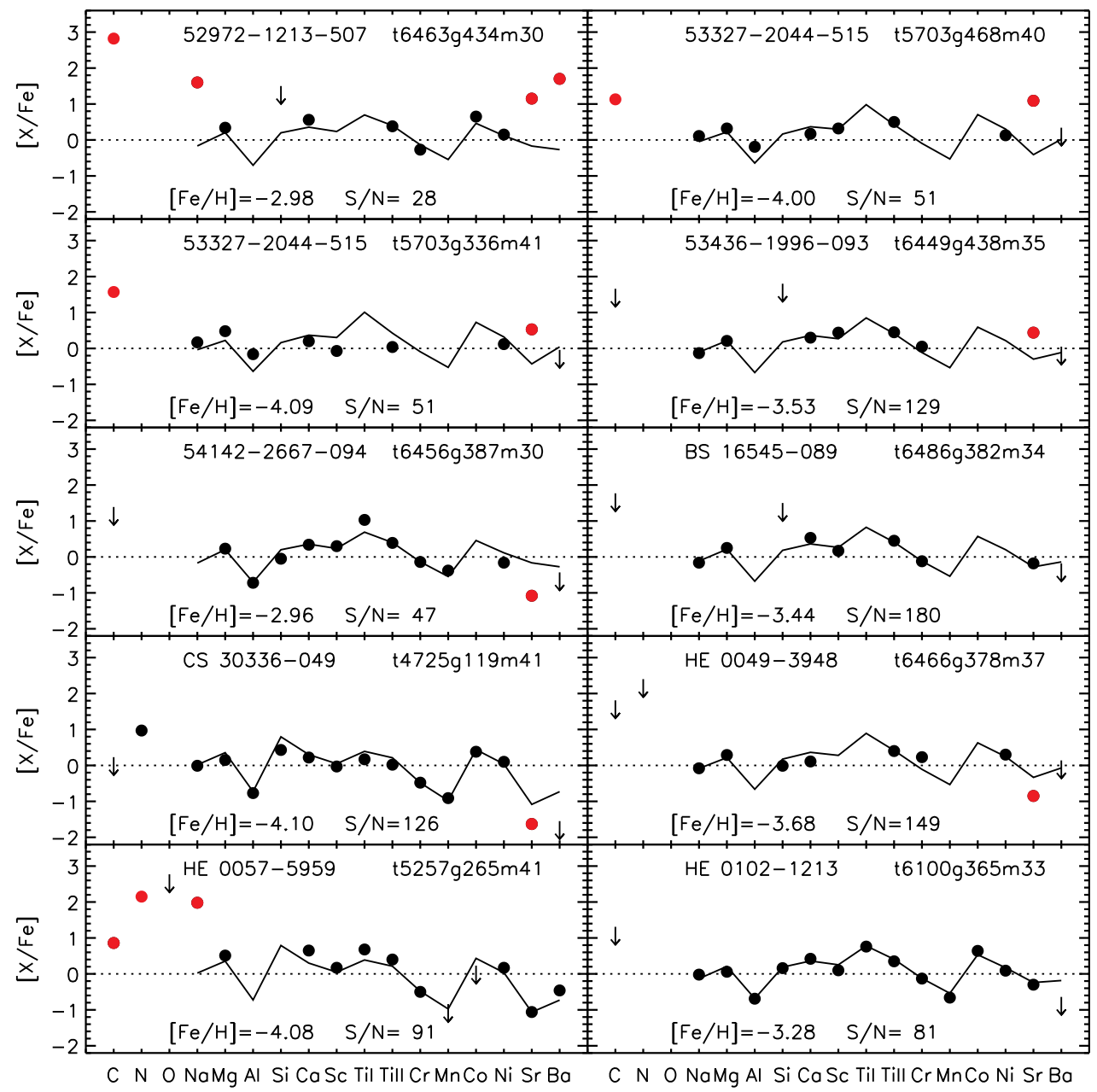

Figure 40. Abundance patterns $[\mathrm{X} / \mathrm{Fe}]$ for each element in our program stars. (Arrows indicate abundance limits.) In each panel, the solid line represents the "normal", $[\mathrm{X} / \mathrm{Fe}]$ abundance ratio for a giant (or dwarf) at the metallicity of each program star (see the text for details). The $\mathrm{S} / \mathrm{N}$ and model parameters are also shown. Red points are for $[\mathrm{C}, \mathrm{N}, \mathrm{O} / \mathrm{Fe}] \geqslant+1.0$, or when $[\mathrm{X} / \mathrm{Fe}]$ differs from the solid line by more than 0.5 dex.

(A color version of this figure is available in the online journal.)

and the mean abundance. In this case, the elements which are poorly fit include $\mathrm{Na}, \mathrm{Sc}$, and Ti.

Presumably, the Kobayashi et al. (2006) model, or other chemical evolution models, could be fine tuned to provide a better fit to this set of observations. We note in particular that the inclusion of yields from intermediate-mass stars cannot account for the underproduction of $\mathrm{Na}$ at low metallicity (Kobayashi et al. 2011b). As discussed earlier, we caution that non-LTE and $3 \mathrm{D}$ effects should be taken into consideration. Chemodynamical models of Galactic formation and evolution (e.g., Kobayashi \& Nakasato 2011), and/or inhomogeneous chemical enrichment models (e.g., Karlsson \& Gustafsson 2005) will enable more comprehensive comparisons with the available observations, including the predicted dispersion in abundance ratios as a function of stellar population characteristics such as metallicity, kinematics, age, and spatial distribution.

\subsection{Chemically Unusual Stars}

In Figures 40-44, we plot for each program star the abundance pattern $[\mathrm{X} / \mathrm{Fe}]$ versus element. (In these figures, we include, as double entries, results of both the dwarf and subgiant analyses of the nine stars for which there was disagreement between spectrophotometric and Balmer-line analyses of the evolutionary status.) The solid line in each panel represents the abundance ratio $[\mathrm{X} / \mathrm{Fe}]$ that a "normal" dwarf $(\log g>3.0)$ or giant $(\log g<3.0)$ star would have at the metallicity of the program star. The "normal" star abundance was taken from the linear trends described above, and plotted in Figures 17-30. For elements that deviate from the solid line by more than $0.50 \mathrm{dex}$, we regard these abundances to be peculiar, and mark them in red. (Note that some peculiar abundances lie above the solid line, while others lie below.) For CEMP objects, we mark their $\mathrm{C}$ abundance in red. For $\mathrm{N}$ and $\mathrm{O}$, we regard ratios $[\mathrm{X} / \mathrm{Fe}] \geqslant$ +1.0 to be unusual, and also mark them in red. Inspection of Figures 40-44 then readily highlights whether a given star has elements that may be regarded as peculiar. Any star with several such elements can be considered as a chemically peculiar star.

Similarly, we examined the 152 star literature sample in order to identify the chemically peculiar objects. In Figures 45-47, we plot the abundance pattern $[\mathrm{X} / \mathrm{Fe}]$ versus element for the subset of program stars with $[\mathrm{Fe} / \mathrm{H}] \leqslant-2.55$ in which there are at least two elements that are unusual (i.e., elements that are at least 0.5 dex above, or below, the $[\mathrm{X} / \mathrm{Fe}]$ ratio of a "normal" dwarf or giant at the same $[\mathrm{Fe} / \mathrm{H}])$. For the two most Fe-poor stars in these figures, the solid lines showing the $[\mathrm{X} / \mathrm{Fe}]$ ratios of a normal star are for $[\mathrm{Fe} / \mathrm{H}]=-4.2$. 


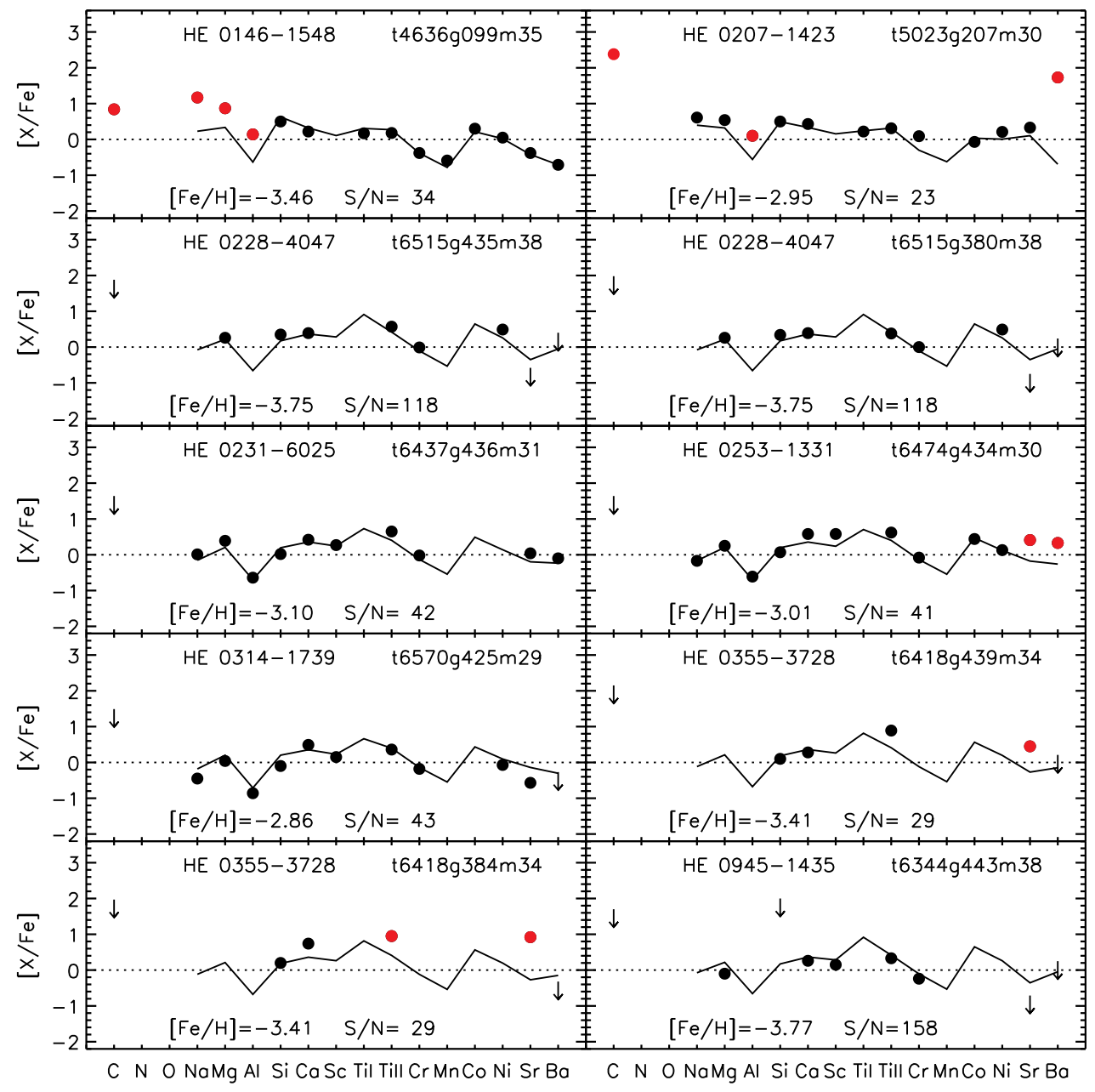

Figure 41. Same as Figure 40, but for the next 10 stars.

(A color version of this figure is available in the online journal.)

In Section 7.2, we noted that in the $[\mathrm{X} / \mathrm{Fe}]$ versus $[\mathrm{Fe} / \mathrm{H}]$ plane, abundance outliers were often, but not always, CEMP objects. We now comment briefly on the C-normal population (i.e., only those stars with $[\mathrm{C} / \mathrm{Fe}]$ measurements, or limits, that enable us to confirm that they are not CEMP stars). We find that of the $109 \mathrm{C}$-normal stars, some 32 are chemically peculiar (i.e., these 32 stars have at least one element, from $\mathrm{Na}$ to $\mathrm{Ba}$, for which the $[\mathrm{X} / \mathrm{Fe}]$ ratio is at least 0.5 dex above, or below, that of a normal star at the same metallicity). These 32 objects have a mean metallicity of $[\mathrm{Fe} / \mathrm{H}]=-3.02$. If we exclude the neutron-capture elements $\mathrm{Sr}$ and $\mathrm{Ba}$ (these elements exhibit a very large dispersion such that many stars will have $[\mathrm{X} / \mathrm{Fe}]$ ratios at least 0.5 dex from the "normal" star abundance), there are $23 \mathrm{C}$-normal stars that are chemically peculiar, and these stars have a mean metallicity of $[\mathrm{Fe} / \mathrm{H}]=-2.91$.

We note that for the majority of these objects, only one element in a given star may be regarded as unusual. If we consider only those C-normal stars that are chemically peculiar for two or more elements, there are seven such objects with a mean metallicity of $[\mathrm{Fe} / \mathrm{H}]=-3.31$. When excluding $\mathrm{Sr}$ and $\mathrm{Ba}$, there are only four $\mathrm{C}$-normal stars, with an average metallicity of $[\mathrm{Fe} / \mathrm{H}]=-3.14$, that are chemically peculiar for two or more elements-HE 1207-3108 (This Study), HE 0024-2523 (Carretta et al. 2002; Cohen et al. 2002), HE 1424-0241 (Cohen et al. 2008), and CS 22873-055 (Cayrel et al. 2004). The main point of this analysis is to note that indeed there are C-normal objects that are chemically peculiar, although the fraction is small, four of 109 objects $(4 \% \pm 2 \%)$. We now discuss some interesting examples of chemically unusual stars.

\subsubsection{Stars with Enhanced $\mathrm{C}, \mathrm{N}, \mathrm{O}, \mathrm{Na}, \mathrm{Mg}$, and/or $\mathrm{Al}$}

HE 0057-5959, HE 1506-0113, and HE 2139-5432 are extremely metal-poor stars, $[\mathrm{Fe} / \mathrm{H}] \leqslant-3.5$, with large enhancements of $\mathrm{C}, \mathrm{N}, \mathrm{O}, \mathrm{Na}, \mathrm{Mg}$, and/or Al. All are CEMP-no objects, i.e., they are a subclass of CEMP stars that have "no strong overabundances of neutron-capture elements," $[\mathrm{Ba} / \mathrm{Fe}]<0$ (Beers \& Christlieb 2005, p. 538). HE 1506-0113 and HE 2139-5432 bear a striking resemblance to HE 1327-2326, the most Fe-poor star known (Frebel et al. 2005; Aoki et al. 2006), as well as to the CEMP-no stars CS 22949-037 and CS 29498-043 (e.g., Aoki et al. 2004). HE 0057-5959 appears to be an extremely rare nitrogen-enhanced metal-poor star (NEMP; Johnson et al. 2007; Pols et al. 2012). Johnson et al. (2007) identified only four stars in the recent literature that could potentially be classified as NEMP objects. HE 0146-1548 also exhibits enhancements of $\mathrm{C}, \mathrm{Na}, \mathrm{Mg}$, and $\mathrm{Al}$. In Paper IV of this series (Norris et al. 2013b), we shall explore the nature of the CEMP-no objects in more detail. 


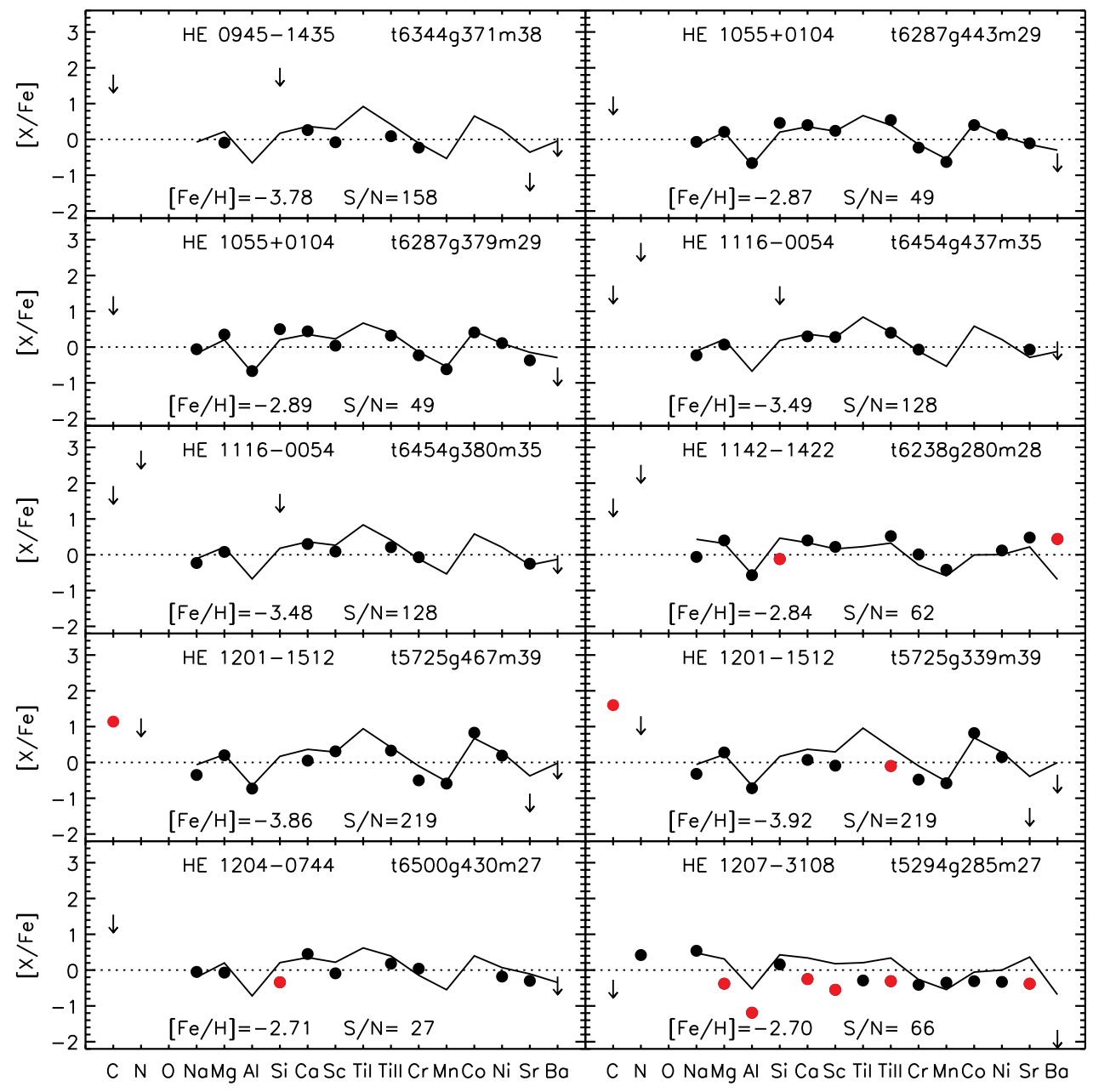

Figure 42. Same as Figure 40, but for the next 10 stars.

(A color version of this figure is available in the online journal.)

\subsubsection{A Star with Enhanced Si}

HE 2136-6030 is a C-normal star with an unusually high Si abundance, $[\mathrm{Si} / \mathrm{Fe}]=+1.20$. However, for all other elements measured, the abundance ratios are in good agreement with a "normal" star at the same metallicity. This object has $T_{\text {eff }}=$ $6409 \mathrm{~K}$ and $[\mathrm{Fe} / \mathrm{H}]=-2.88$. Figure 20 shows that for dwarf stars, the $[\mathrm{Si} / \mathrm{Fe}]$ ratio is almost constant as $[\mathrm{Fe} / \mathrm{H}]$ evolves from -4.0 to -2.5 . While Figure 32 shows a strong trend between $[\mathrm{Si} / \mathrm{Fe}]$ and $T_{\text {eff }}$, stars with $T_{\text {eff }}$ similar to that of HE 2136-6030 all have $[\mathrm{Si} / \mathrm{Fe}] \lesssim+0.5$ dex. In Figure 48, we plot the spectra of HE 2136-6030, along with two Si-normal stars with similar stellar parameters. The Si line is substantially stronger in HE 2136-6030, relative to the two comparison stars. Examination of our spectra shows that $\mathrm{CH}$ blending of the $3905.52 \AA \mathrm{Si}$ line is unlikely, and that the subordinate $4102.94 \AA \mathrm{Si}$ line is likely present. Spectrum synthesis of the $4102.94 \AA \mathrm{Si}$ line returns a $[\mathrm{Si} / \mathrm{Fe}]$ ratio in good agreement with the abundance from the $3905.52 \AA$ line. We are thus confident that the $\mathrm{Si}$ abundance is particularly high in this star.

At the other extreme, Cohen et al. (2007) found a star with an unusually low $\mathrm{Si}$ abundance, $[\mathrm{Si} / \mathrm{Fe}]=-1.01$. This star, HE 1424-0241, also has low abundances of $[\mathrm{Ca} / \mathrm{Fe}]$ and $[\mathrm{Ti} / \mathrm{Fe}]$ but a normal $[\mathrm{Mg} / \mathrm{Fe}]$ ratio. These two stars, HE 2136-6030 and HE 1424-0241, reveal that the [Si/Fe] ratio in metal-poor stars can vary by a factor of 100 .

\subsection{3. "Fe-enhanced" Metal-poor Stars}

$\mathrm{HE} 1207-3108\left(T_{\text {eff }} / \log g /[\mathrm{Fe} / \mathrm{H}]=5294 / 2.85 /-2.70\right)$ is notable for having unusually low abundance ratios $[\mathrm{X} / \mathrm{Fe}]$ for $\mathrm{Mg}, \mathrm{Al}, \mathrm{Ca}, \mathrm{Sc}, \mathrm{Ti}$, and $\mathrm{Sr}$. (With the exception of Ti, and possibly $\mathrm{Sr}$, none of these elements exhibit significant trends with $T_{\text {eff }}$, thus it is highly unlikely that the peculiar abundance pattern can be attributed to any $T_{\text {eff }}$-dependent trends.) The effect is shown in Figure 49, where the filled circles represent [X/Fe] for HE 1207-3108 as a function of atomic species. Indeed, one sees here that for all elements other than $\mathrm{Na}$, the abundance ratios $[\mathrm{X} / \mathrm{Fe}]$ lie below that of a "normal" star at the same metallicity. (We note here that the two $\mathrm{Na}$ lines yield abundances that differ by 0.48 dex. Without knowing which of the lines to reject, we retain both lines and the large error reflects the discordant measurements.) Cayrel et al. (2004) identified another star, CS 22169-035, as being deficient in $\mathrm{Mg}, \mathrm{Si}, \mathrm{Ca}, \mathrm{Ti}, \mathrm{Co}, \mathrm{Ni}$, and $\mathrm{Zn}$ with respect to Fe. They suggested that (p. 1135) "the abundance anomalies are most simply characterized as an enhancement of Fe;" the same description may be applied to HE 1207-3108. Inspection of Figure 42 suggests that if the $\mathrm{Fe}$ abundance were lowered, by say 0.6 dex, HE 1207-3108 would have normal abundance ratios $[\mathrm{X} / \mathrm{Fe}]$ for $\mathrm{Mg}$ and all heavier elements, but $[\mathrm{Na} / \mathrm{Fe}]$ might be regarded as being unusually high.

In Figure 49, we also present the data for CS 22169-035. Here, we we arbitrarily select Sc as the element to which we 


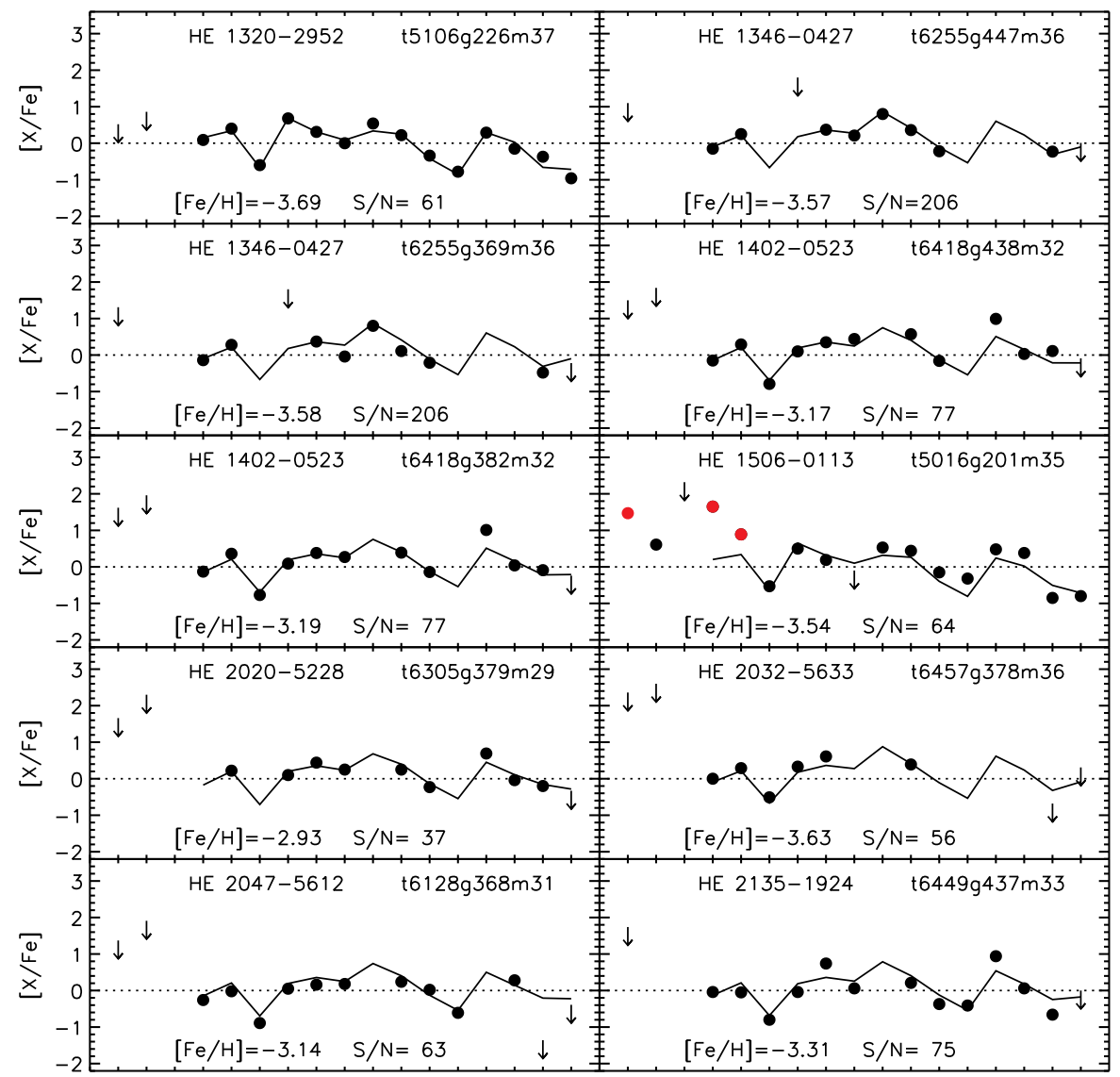

C N O NaMg Al Si CaSc Til Till CrMnCo Ni Sr Ba C N O NaMg Al Si CaSe Til Till Cr MnCo Ni Sr Bo

Figure 43. Same as Figure 40, but for the next 10 stars.

(A color version of this figure is available in the online journal.)

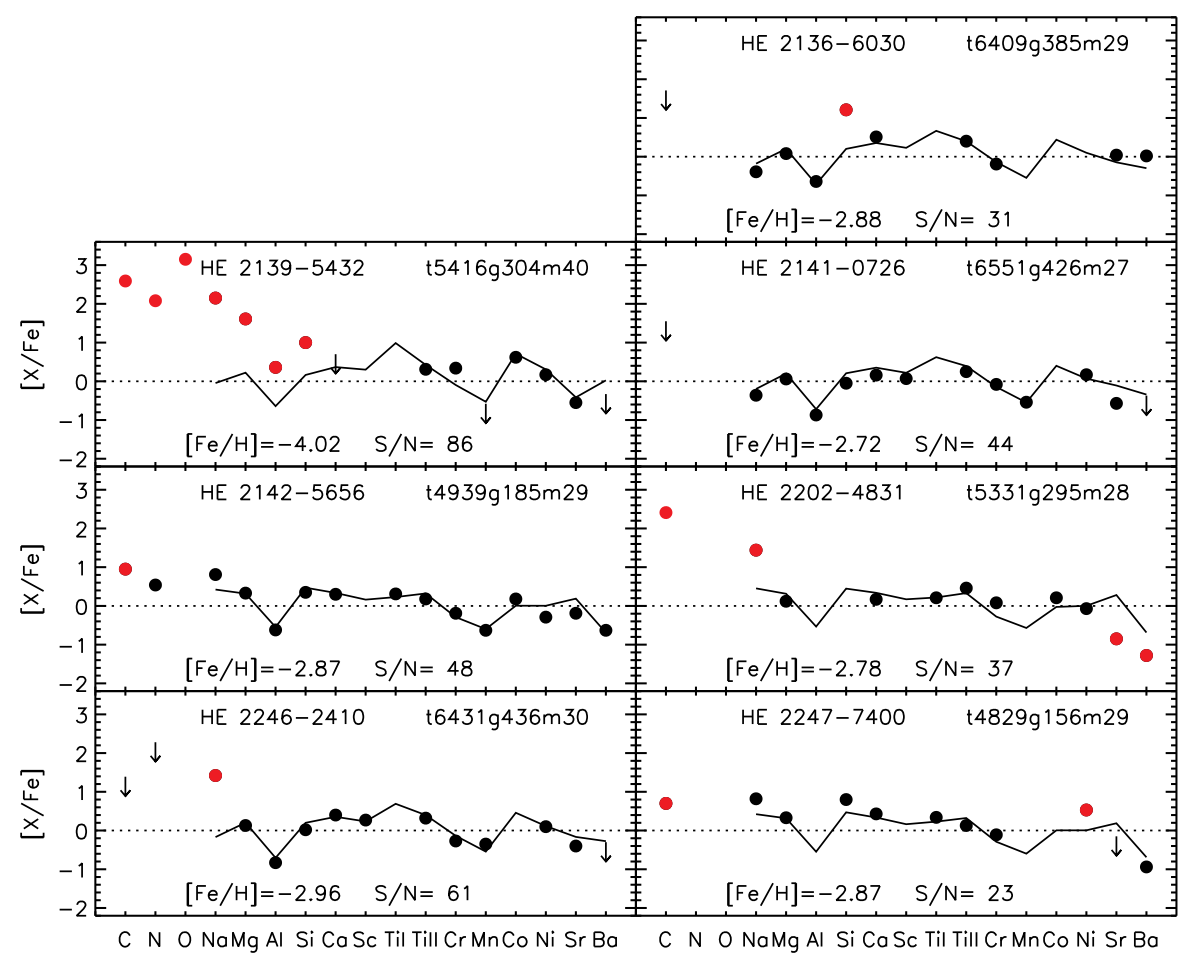

Figure 44. Same as Figure 40, but for the final seven stars.

(A color version of this figure is available in the online journal.) 


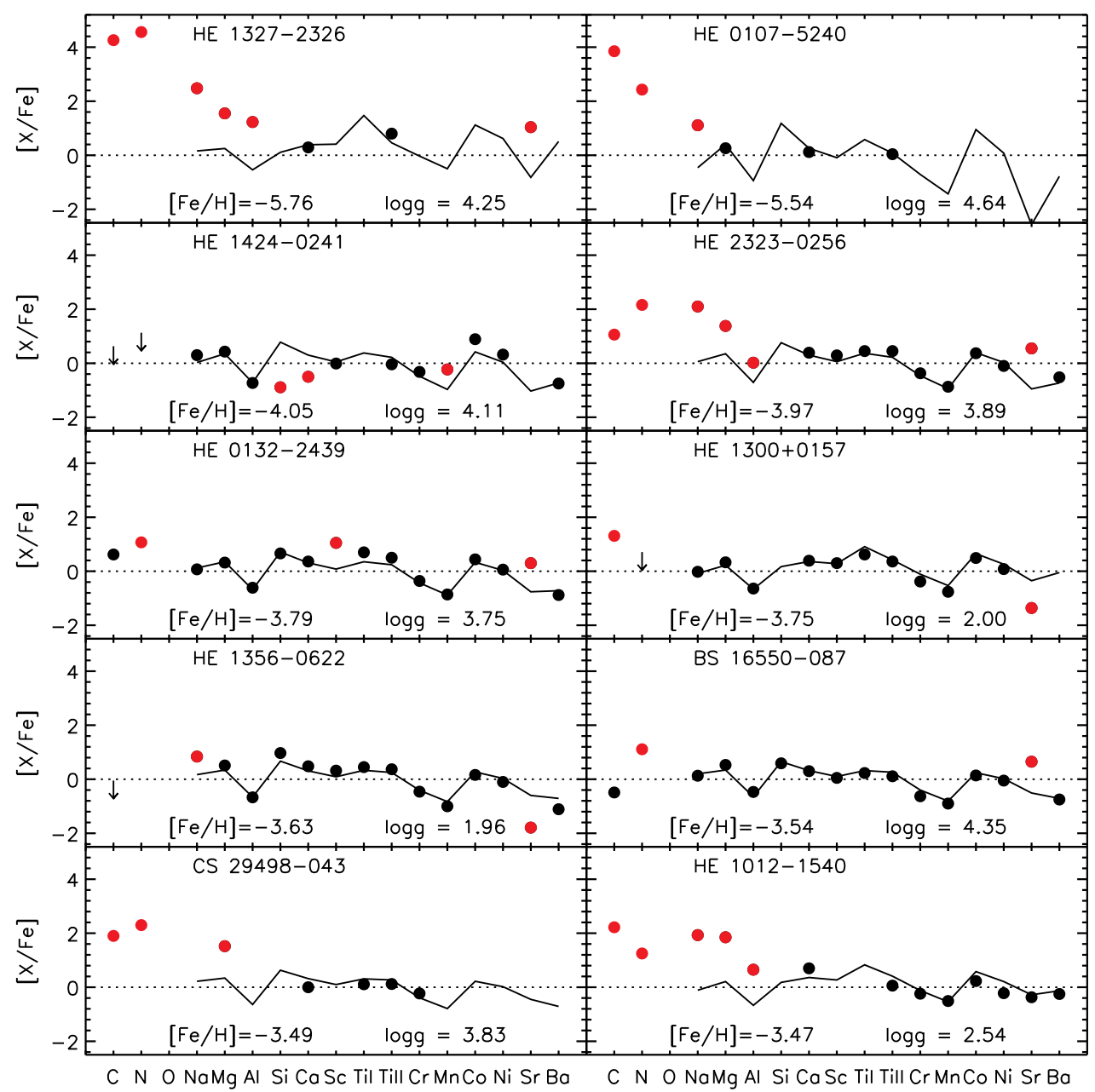

Figure 45. Same as Figure 40, but for the literature sample. The stars are ordered by increasing metallicity, and only stars with [Fe/H] $\leqslant-2.55$ and with two or more unusual elements are plotted. For the two most Fe-poor stars, the "normal" $[\mathrm{X} / \mathrm{Fe}]$ abundance ratios are for $[\mathrm{Fe} / \mathrm{H}]=-4.2$ (see the text for details).

(A color version of this figure is available in the online journal.)

normalize the abundances; this requires a shift of -0.47 dex for CS 22169-035. The solid line shows the abundance ratio that a "normal" giant would have at the metallicity of HE 1207-3108. (An equivalent line for CS 22169-035 would be essentially identical, given that both stars are giants with comparable metallicity, $[\mathrm{Fe} / \mathrm{H}]=-2.70$ and -2.95 for $\mathrm{HE} 1207-3108$ and $\mathrm{CS}$ 22169-035, respectively.) HE 1207-3108 and CS 22169-035 appear to have similar and unusual abundance patterns, i.e., they are chemically distinct from the bulk of the halo stars at the same metallicity. We note that their chemical abundance patterns are not identical, namely, there are differences for $\mathrm{Cr}, \mathrm{Co}, \mathrm{Ni}$, and $\mathrm{Sr}$. That said, their abundance ratios for $\mathrm{Mg}, \mathrm{Al}, \mathrm{Ca}, \mathrm{Ti}$, and $\mathrm{Mn}$ are indistinguishable and unusually low. We suggest that HE 1207-3108 and CS 22169-035 may belong to a relatively new and growing class of "Fe-enhanced metal-poor" stars.

A small number of field halo stars, both dwarfs and giants, are known to have low $[\alpha / \mathrm{Fe}]$ ratios relative to field stars at the same metallicity (e.g., Carney et al. 1997; Nissen \& Schuster 1997; Fulbright 2002; Stephens \& Boesgaard 2002; Ivans et al. 2003; Nissen \& Schuster 2010). These stars cover the metallicity range $-2 \lesssim[\mathrm{Fe} / \mathrm{H}] \lesssim-0.8$. One suggestion is that these stars likely formed from regions in which the interstellar gas was unusually enriched in the products of Type Ia supernovae, relative to
Type II supernovae, and as such they may be regarded as having an unusually high Fe-content relative to their $\alpha$-content. One explanation is that these stars may have been accreted from dwarf galaxies, many of which are known to contain stars that are chemically distinct from the majority of field halo stars (e.g., Tolstoy et al. 2009). While it may be tempting to assign the low $[\alpha / \mathrm{Fe}]$ stars and HE $1207-3108$ and CS 22169-035 into a single group, there are differences in $[\mathrm{Fe} / \mathrm{H}]$ and other $[\mathrm{X} / \mathrm{Fe}]$ ratios. Nevertheless, this intriguing possibility exists.

Venn et al. (2012) have identified a giant star in the Carina dwarf galaxy with a similar chemical pattern to that of HE 1207-3108 and CS 22169-035. Their star, Car-612, with $[\mathrm{Fe} / \mathrm{H}]=-1.3$, is considerably more metal-rich than HE 1207-3108 and CS 22169-035, with $[\mathrm{Fe} / \mathrm{H}]=-2.70$ and -2.95 , respectively. Venn et al. (2012) suggest that this star formed from gas with an unusually high ratio of Type Ia supernovae to Type II supernovae products. They estimate the magnitude of the "excess of $\mathrm{Fe}$ " to be $0.7 \mathrm{dex}$, a value comparable to that of HE 1207-3108 and CS 22169-035. Had we overplotted their data for this star in Figure 49, again normalizing to the Sc abundances, there would have been striking similarities in the some abundance ratios $(\mathrm{Mg}, \mathrm{Ti}$, and $\mathrm{Ni}$ ), as well as notable differences for other elements (Ca, $\mathrm{Cr}$, and $\mathrm{Mn}$ ). 


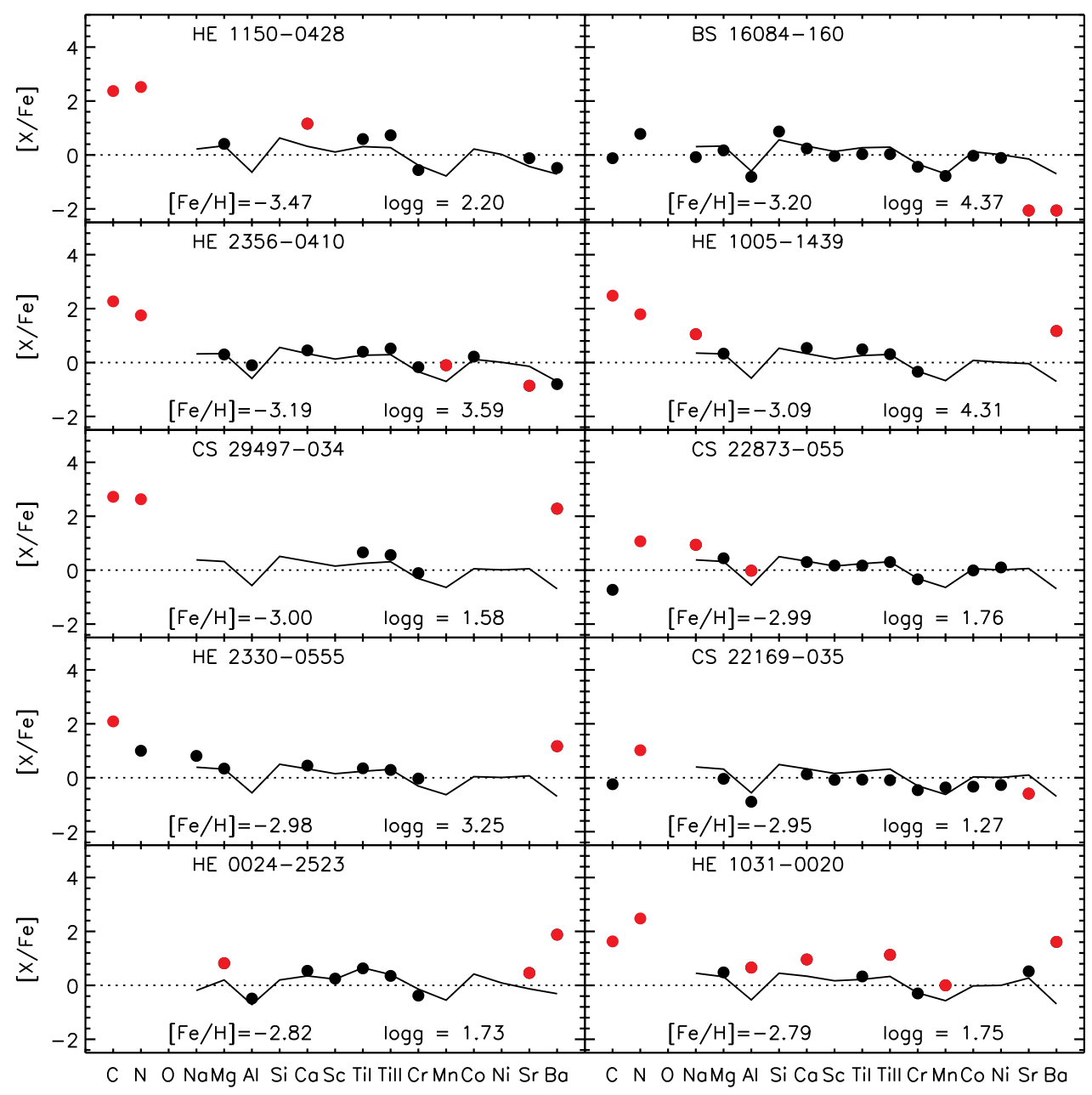

Figure 46. Same as Figure 40, but for the next 10 stars.

(A color version of this figure is available in the online journal.)

As before, we do not overplot these data, since we do not wish to conduct inhomogeneous comparisons. Nevertheless, it is tempting to associate the three stars (HE 1207-3108, CS 22169-035, and Car-612) as belonging to the same class of object. We tentatively speculate that any differences in $[\mathrm{X} / \mathrm{Fe}]$ ratios between Car-612, HE 1207-3108, and CS 22169-035 may reflect differences in the currently unknown formation processes for such stars. (During publication, we were informed of another "Feenhanced metal-poor star" discovered by Cohen \& Huang 2010 in the Ursa Minor dwarf spheroidal galaxy.)

\subsubsection{A CEMP-s Star with Enhanced [Ba/Sr]}

HE 0207-1423 $([\mathrm{Fe} / \mathrm{H}]=-2.95)$ is a CEMP-s star with the unusually large $\mathrm{Ba} / \mathrm{Sr}$ ratio of $[\mathrm{Ba} / \mathrm{Sr}]=+1.40$. (CEMP-s stars are a subclass of CEMP objects that exhibit overabundances of the $s$-process elements, defined by $[\mathrm{Ba} / \mathrm{Fe}]>+1.0$ and $[\mathrm{Ba} / \mathrm{Eu}]>+0.5$; Beers \& Christlieb 2005.) That is to say, the nucleosynthetic site(s) that produced the large $\mathrm{Ba}$ abundance, $[\mathrm{Ba} / \mathrm{Fe}]=+1.73$, in this object, yielded only $[\mathrm{Sr} / \mathrm{Fe}]=+0.33$, considerably smaller than one might have expected. Inspection of Figure 50 presents the dependence of $[\mathrm{Ba} / \mathrm{Sr}]$ on $[\mathrm{Fe} / \mathrm{H}]$ (which we shall discuss in the following section), and shows that there are other (literature) stars that exhibit unusually large $[\mathrm{Ba} / \mathrm{Sr}]$ values (or low $[\mathrm{Sr} / \mathrm{Ba}]$ ). These stars are all CEMP stars with $[\mathrm{Fe} / \mathrm{H}] \geqslant-3.0$.

\subsection{Neutron-capture Elements and CEMP Stars}

Although our spectra are of moderate-to-high quality, we were only able to measure the abundance of two neutron-capture elements, $\mathrm{Sr}$ and $\mathrm{Ba}$. The absence of other neutron-capture elements was not for lack of effort: in the course of our analysis, we tried to measure the equivalent widths of numerous lines of La II, Ce II, Nd II, and Eu II. That said, our measurements of $\mathrm{Sr}$ and $\mathrm{Ba}$ enable us to comment on three issues.

First, previous studies have found a very large scatter in $\mathrm{Sr}$ and $\mathrm{Ba}$ abundances at low metallicity. In Figures 29 and 30, there is a large dispersion about the linear fit to the $[\mathrm{Sr} / \mathrm{Fe}]$ versus $[\mathrm{Fe} / \mathrm{H}]$ and $[\mathrm{Ba} / \mathrm{Fe}]$ versus $[\mathrm{Fe} / \mathrm{H}]$ trends, for both dwarfs and giants, even after excluding CEMP stars and $2 \sigma$ outliers. In all four cases, the dispersion (ranging from 0.30 dex to $0.60 \mathrm{dex}$ ) exceeds the typical measurement uncertainties (ranging from 0.15 dex to 0.22 dex). In Figure 50, we consider [Ba/Sr] versus $[\mathrm{Fe} / \mathrm{H}]$. For dwarfs, the dispersion about the linear fit to the data (with the usual exclusions) is $0.28 \mathrm{dex}$. This is comparable to the dispersions for $[\mathrm{Sr} / \mathrm{Fe}](0.36$ dex, Figure 29$)$ and $[\mathrm{Ba} / \mathrm{Fe}]$ (0.30 dex, Figure 30). For giants, the dispersion about the linear fit to $[\mathrm{Ba} / \mathrm{Sr}]$ versus $[\mathrm{Fe} / \mathrm{H}]$ is 0.56 dex. Again, the value is comparable to the dispersions for $[\mathrm{Sr} / \mathrm{Fe}](0.60 \mathrm{dex}$, Figure 29) and $[\mathrm{Ba} / \mathrm{Fe}](0.43 \mathrm{dex}$, Figure 30$)$. Therefore, our data set suggests that the dispersion about the linear fit to $[\mathrm{Ba} / \mathrm{Sr}]$ versus 


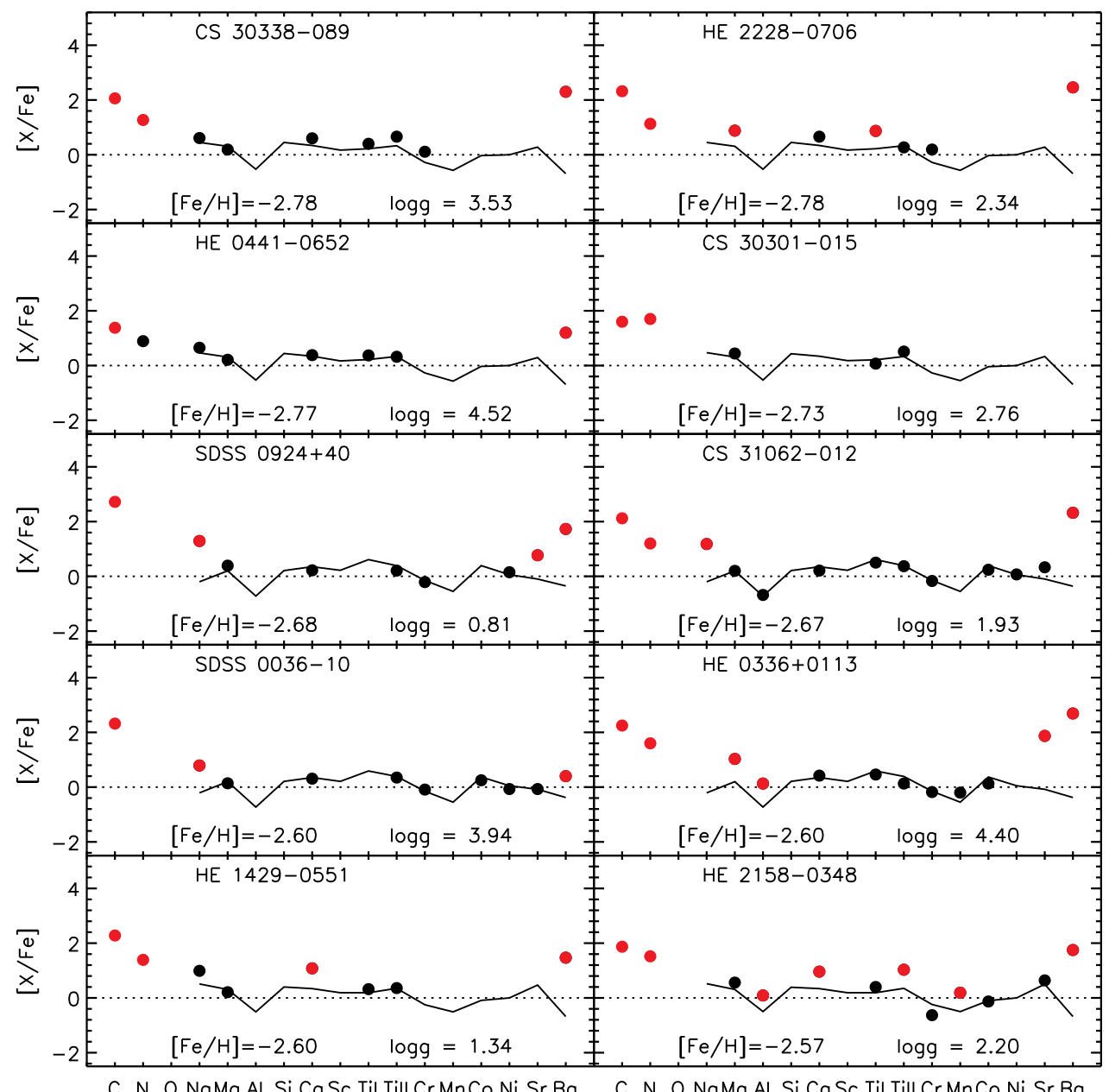

Figure 47. Same as Figure 40, but for the final 10 stars.

(A color version of this figure is available in the online journal.)

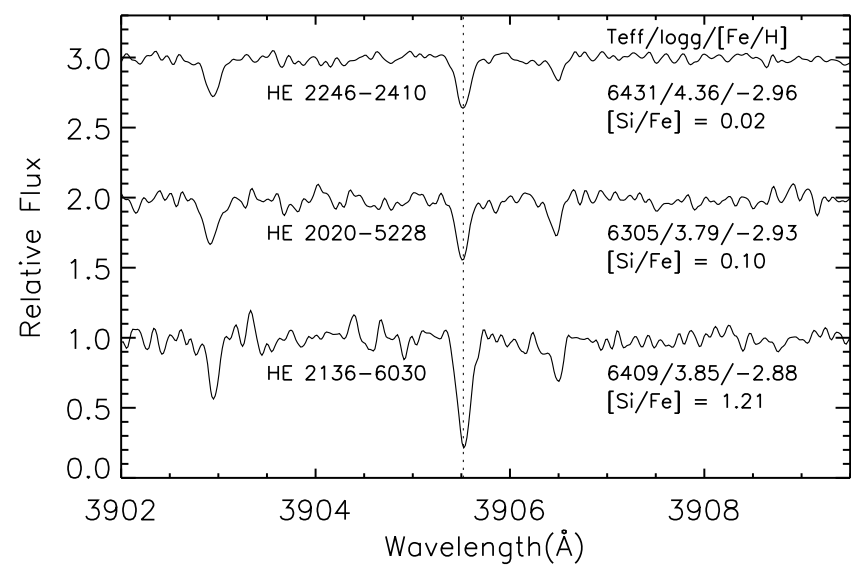

Figure 48. Spectra in the region of the $3905 \AA \mathrm{Si}$ line for two Si-normal stars (HE 2246-2410 and HE 2020-5228), and for the Si-enhanced star (HE 2136-6060). The $T_{\text {eff }}, \log g,[\mathrm{Fe} / \mathrm{H}]$, and $[\mathrm{Si} / \mathrm{Fe}]$ are displayed.

$[\mathrm{Fe} / \mathrm{H}]$ is not smaller than the individual dispersions about the linear fit to $[\mathrm{Sr} / \mathrm{Fe}]$ or $[\mathrm{Ba} / \mathrm{Fe}]$ versus $[\mathrm{Fe} / \mathrm{H}]$, as might have been expected if a correlation existed between the abundances of the two elements.

Second, there is evidence that an additional process (or processes) dominates the production of the lighter neutron-capture elements. François et al. (2007) plotted [(Sr, Y, Zr)/Ba]

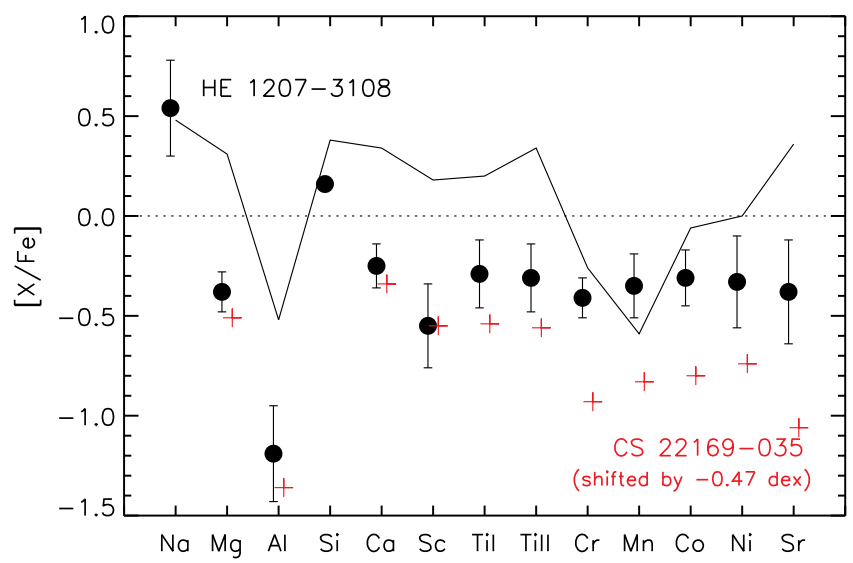

Figure 49. [X/Fe] for the two "Fe-enhanced" metal-poor stars HE 1207-3108 (black circles) and CS 22169-035 (red plus signs). By normalizing the abundances to Sc, we shift the abundance ratios for CS $22169-035$ by -0.43 dex. The solid line represents the "normal" $[\mathrm{X} / \mathrm{Fe}]$ ratio for a giant at the metallicity of HE 1207-3108.

(A color version of this figure is available in the online journal.)

versus $[\mathrm{Ba} / \mathrm{H}]$ (see their Figure 15), and noted that (1) for $[\mathrm{Ba} / \mathrm{H}] \geqslant-2.5$, all ratios are close to the solar value, $(2)$ for $-4.5 \leqslant[\mathrm{Ba} / \mathrm{H}] \leqslant-2.5[(\mathrm{Sr}, \mathrm{Y}, \mathrm{Zr}) / \mathrm{Ba}]$ increases as $[\mathrm{Ba} / \mathrm{H}]$ decreases, and $(3)$ for $[\mathrm{Ba} / \mathrm{H}] \leqslant-4.5$, all ratios drop to solar. 

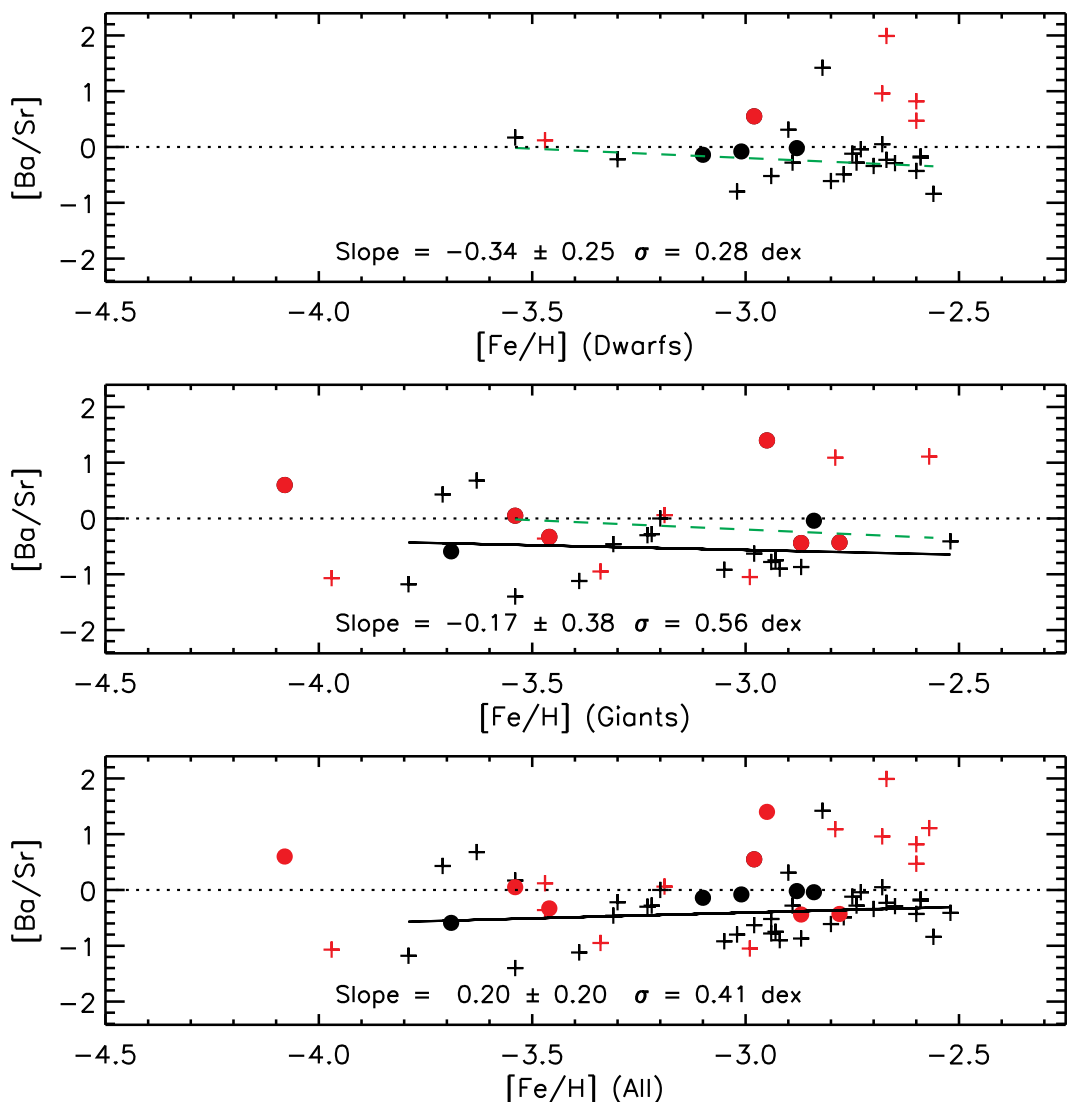

Figure 50. [Ba/Sr] vs. [Fe/H] for dwarfs (upper), giants (middle), and all stars (lower). The symbols are the same as in Figure 15. In each panel we present the linear fit to the data (excluding CEMP stars and $2 \sigma$ outliers). The linear fit to the dwarf data is superimposed upon the giant data. The slopes and uncertainties are shown, along with the dispersion about the slope.

(A color version of this figure is available in the online journal.)
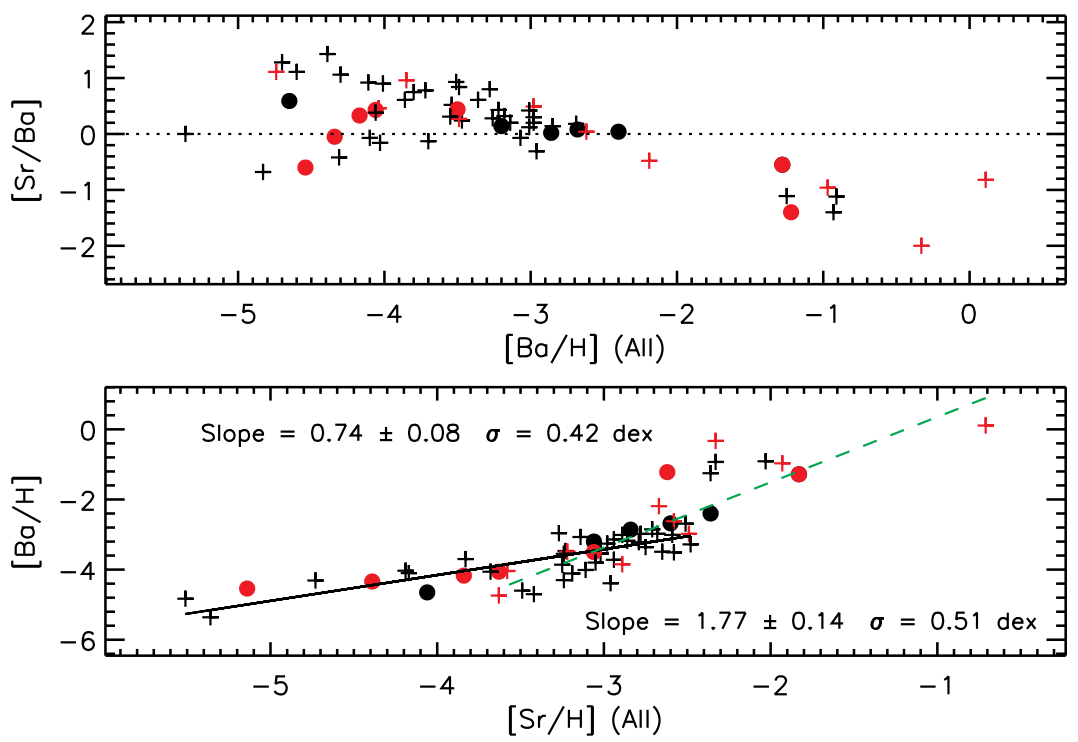

Figure 51. $[\mathrm{Sr} / \mathrm{Ba}]$ vs. $[\mathrm{Ba} / \mathrm{H}]$ (upper) and $[\mathrm{Ba} / \mathrm{H}]$ vs. $[\mathrm{Sr} / \mathrm{H}]$ (lower). The symbols are the same as in Figure 15 . In the lower panel, we present the linear fit to the data (excluding $2 \sigma$ outliers) with $[\mathrm{Sr} / \mathrm{H}] \leqslant-2.4$ (solid black line) and $[\mathrm{Sr} / \mathrm{H}] \geqslant-3.65$ (dashed green line). For the two lines, the slopes and uncertainties are shown, along with the dispersion about the slope.

(A color version of this figure is available in the online journal.) 

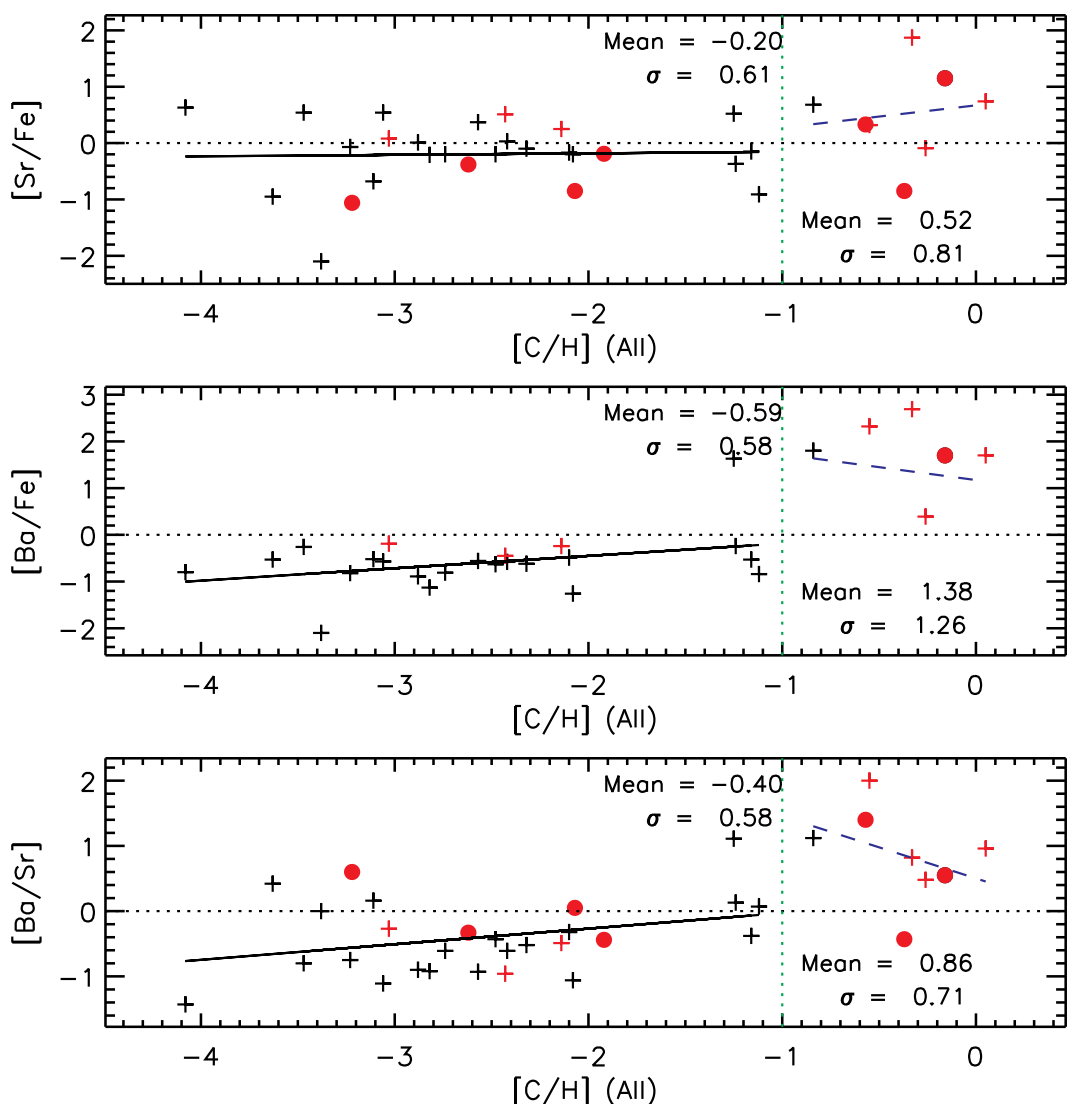

Figure 52. $[\mathrm{Sr} / \mathrm{Fe}]$ (upper), $[\mathrm{Ba} / \mathrm{Fe}]$ (middle), and [Ba/Sr] (lower) vs. $[\mathrm{C} / \mathrm{H}]$. The symbols are the same as in Figure 15 . (A color version of this figure is available in the online journal.)

They concluded that such observations are inconsistent with a single $r$-process. In Figure 51 (upper panel), we consider the ratio $[\mathrm{Sr} / \mathrm{Ba}]$ versus $[\mathrm{Ba} / \mathrm{H}]$. Our data are qualitatively consistent with those of François et al. (2007) and their results, had we overplotted them, would have provided a very good match to ours. However, such an inhomogeneous comparison would be contrary to the spirit of the homogeneous analysis presented herein. Considering only our data below $[\mathrm{Ba} / \mathrm{H}]=$ -2.5 , the dispersion in $[\mathrm{Sr} / \mathrm{Ba}]$ appears to increase with decreasing $[\mathrm{Ba} / \mathrm{H}]$. Indeed, there is a hint of two populations below $[\mathrm{Ba} / \mathrm{H}]=-4.0$, one with high $[\mathrm{Sr} / \mathrm{Ba}]$ and the other with solar, or sub-solar, [Sr/Ba], although the statistics are still relatively poor.

The lower panel of Figure 51 shows $[\mathrm{Ba} / \mathrm{H}]$ versus $[\mathrm{Sr} / \mathrm{H}]$. Within the limited data, there is a hint of two (overlapping) populations, with the boundary at $[\mathrm{Sr} / \mathrm{H}] \simeq-3.2$. Excluding $2 \sigma$ outliers (and in this case retaining CEMP stars), we find that the two populations have significantly different slopes, but very similar dispersions about the linear fit. These data may indicate that at lowest $\mathrm{Sr}$ and $\mathrm{Ba}$ abundances, the nucleosynthetic process(es) that create $\mathrm{Sr}$ and $\mathrm{Ba}$ produces a different ratio of $\mathrm{Sr} / \mathrm{Ba}$ than the nucleosynthetic process(es) that operate when the $\mathrm{Sr}$ and $\mathrm{Ba}$ abundances are higher. The scaled-solar $r$-process distribution would be represented by a line of gradient 1.00 in the $[\mathrm{Sr} / \mathrm{H}]$ versus $[\mathrm{Ba} / \mathrm{H}]$ plane. For the two populations that we identify, neither slope matches the main $r$-process line, which may suggest that there are two (or more) nucleosynthetic sites producing $\mathrm{Sr}$ and $\mathrm{Ba}$ at low metallicity that are distinct from the main $r$-process. Ignoring the sole point with $[\mathrm{Sr} / \mathrm{H}] \geqslant$
-1.5 , one might still argue that the data with highest $[\mathrm{Sr} / \mathrm{H}]$ (admittedly mainly CEMP objects) do not appear to lie on a linear extrapolation of the line fitting the data with lower $[\mathrm{Sr} / \mathrm{H}]$. Again, we note in passing that the François et al. (2007) data confirm the trends seen in our data. Had we included their measurements in the linear fits to the data described above, we would have obtained essentially identical slopes, uncertainties, and dispersions about the linear fits. However, once again we do not overplot these data to avoid inhomogeneous comparisons. Travaglio et al. (2004) noted the need for a primary process to produce $\mathrm{Sr}, \mathrm{Y}$, and $\mathrm{Zr}$ at low metallicity that was different from the $s$ - and the $r$-process. They referred to this as a lighter element primary process (LEPP). At face value, our results are consistent with the suggestion there may be two components to the Travaglio et al. (2004) LEPP. Roederer et al. (2010) suggest that high-entropy neutrino winds from core-collapse supernovae can explain the diversity of neutron-capture element abundances found at low metallicity. It would be interesting to examine whether our $\mathrm{Sr}$ and $\mathrm{Ba}$ measurements can be explained by this scenario.

Third, the relation between the abundances of $\mathrm{C}$ and the neutron capture elements $\mathrm{Ba}$ and $\mathrm{Sr}$ may shed light on the process(es) that created the CEMP-s and CEMP-no classes of stars. As already noted, Beers \& Christlieb (2005) defined CEMP-s stars as those with $[\mathrm{C} / \mathrm{Fe}] \geqslant+1.0,[\mathrm{Ba} / \mathrm{Fe}]>+1.0$, and $[\mathrm{Ba} / \mathrm{Eu}]>+0.5$, and CEMP-no stars as those with $[\mathrm{C} / \mathrm{Fe}] \geqslant$ +1.0 and $[\mathrm{Ba} / \mathrm{Fe}]<0$. The CEMP-s stars are the majority population, $\sim 80 \%$ of all CEMP objects (Aoki 2010), and radial-velocity studies have revealed that the observed binary 
frequency in this subclass is consistent with a binary fraction of $100 \%$ (Lucatello et al. 2005). Mass transfer between an asymptotic giant branch (AGB) primary onto the currently CEMP-s star is believed to be responsible for the $\mathrm{C}$ and $\mathrm{Ba}$ enhancements (see, e.g., Izzard et al. 2009 for a detailed examination of the viability of this scenario), a process that also produces the more metal-rich classes of $\mathrm{CH}$ and $\mathrm{Ba}$ stars. Additionally, the metallicity distributions differ, with CEMP-s being generally more metal rich, $[\mathrm{Fe} / \mathrm{H}]>-3.0$, and CEMP-no being generally more metal poor, $[\mathrm{Fe} / \mathrm{H}]<-3.0$ (e.g., Cohen et al. 2006; Aoki et al. 2007; Aoki 2010). Cohen et al. (2006) and Masseron et al. (2010) have suggested that the CEMP-no stars are likely the extremely metal-poor counterparts of the CEMP-s stars.

Figure 52 shows $[\mathrm{Sr} / \mathrm{Fe}],[\mathrm{Ba} / \mathrm{Fe}]$, and $[\mathrm{Ba} / \mathrm{Sr}]$ versus $[\mathrm{C} / \mathrm{H}]$. In this figure, the CEMP stars with the highest $[\mathrm{Sr} / \mathrm{Fe}]$ and $[\mathrm{Ba} / \mathrm{Fe}]$ ratios tend to have the highest $[\mathrm{C} / \mathrm{H}]$ values. While more data are urgently needed, we speculate that the stars which produce CEMP objects with $[\mathrm{C} / \mathrm{H}] \geqslant-1.0$ synthesize large amounts of $\mathrm{Sr}$ and $\mathrm{Ba}$. The diverse set of abundance ratios seen in the observations calls for production sites capable of diverse yields. Yields of $\mathrm{C}$ and $s$-process elements from AGB stars are strongly dependent on mass and metallicity, such that they may be good candidates for explaining part, or perhaps most, of the large range of $\mathrm{C}$ and $s$-process element abundances (e.g., Karakas \& Lattanzio 2007; Karakas 2010; Cristallo et al. 2011; Lugaro et al. 2012). Regardless, the limited data indicate that stars with $[\mathrm{C} / \mathrm{H}]>-1.0$ have $\mathrm{Sr}$ and $\mathrm{Ba}$ enhancements and $[\mathrm{Ba} / \mathrm{Sr}]>0$. See Papers III and IV in this series for additional discussions of CEMP stars.

\section{CONCLUDING REMARKS}

We present a homogeneous chemical abundance analysis of 16 elements in 190 metal-poor stars, which comprise 38 program stars and 152 literature stars. The sample includes 86 extremely metal-poor stars, $[\mathrm{Fe} / \mathrm{H}] \leqslant-3.0$, and 10 new stars with $[\mathrm{Fe} / \mathrm{H}] \leqslant-3.5$. To our knowledge this represents one of the largest homogeneous chemical abundance analyses of extremely metal-poor stars based on a model atmosphere analysis of equivalent widths measured in high-resolution, high-S/N spectra.

We find strong evidence for large chemical diversity at low metallicity. For a given abundance ratio, $[\mathrm{X} / \mathrm{Fe}]$, the outliers are often, but not always, CEMP objects. Considering dwarfs and giants separately, we define the linear fit to $[\mathrm{X} / \mathrm{Fe}]$ versus $[\mathrm{Fe} / \mathrm{H}]$ excluding CEMP stars and $2 \sigma$ outliers. We regard these trends as defining the "normal" population of metalpoor stars. For many elements, the dispersions about the linear fits are in good agreement with the scatter expected from measurement uncertainties. Therefore, we believe that (1) a "normal" population exists, and (2) that our crude selection criteria were able to identify this population.

For several elements, there are clear discrepancies between dwarfs and giants. The evidence for abundance differences include significantly different slopes in the $[\mathrm{X} / \mathrm{Fe}]$ versus $[\mathrm{Fe} / \mathrm{H}]$ linear fit (Na, Al, Sc, Ti II, Cr, Mn, and Ni) as well as a hint of differences in the mean abundances $(\mathrm{Na}, \mathrm{Si}, \mathrm{Ti}$ I, $\mathrm{Cr}, \mathrm{Co}$, and $\mathrm{Ba}$ ). Similar results were found by Lai et al. (2008) and Bonifacio et al. (2009). Another way to identify abundance differences between dwarfs and giants is to consider $[\mathrm{X} / \mathrm{Fe}]$ versus $T_{\text {eff. }}$. We find statistically significant trends for many elements studied in this work. These effects (differences in the slopes of linear fits to $[\mathrm{X} / \mathrm{Fe}]$ versus $[\mathrm{Fe} / \mathrm{H}]$ between dwarfs and giants, differences in mean abundances between dwarfs and giants, and trends between $[\mathrm{X} / \mathrm{Fe}]$ versus $T_{\text {eff }}$ ) likely signify the presence of non-LTE and/or 3D effects (Asplund 2005). Therefore, we stress the importance of comparing dwarfs with dwarfs and giants with giants as well as caution when comparing abundance trends with nucleosynthesis and/or chemical evolution predictions. Additionally, abundance differences between dwarfs and giants may be due, in part, to radiative levitation and gravitational settling.

Using linear fits between $[\mathrm{X} / \mathrm{Fe}]$ versus $[\mathrm{Fe} / \mathrm{H}]$ for dwarfs and giants, we identified many examples of individual stars with abundance peculiarities, including CEMP-no objects, one star with $[\mathrm{Si} / \mathrm{Fe}]=+1.2$, one with large $[\mathrm{Ba} / \mathrm{Sr}]$, and a star with unusually low $[\mathrm{X} / \mathrm{Fe}]$ for all elements heavier than Na. While many CEMP stars exhibit peculiar abundances for elements other than $\mathrm{C}$, we note that there are chemically peculiar stars which are not CEMP objects. We find a hint for two nucleosynthetic processes for the production of $\mathrm{Sr}$ and $\mathrm{Ba}$ at lowest metallicity, neither of which match the main $s$-process or $r$-process.

Although the present sample is substantial, there is clear need for considerably more data at the lowest metallicities. Further mining of existing data sets (HK, HES, SDSS, etc.) as well as new and upcoming surveys and facilities (e.g., SkyMapper, Keller et al. 2007; LAMOST, Zhao et al. 2006) should increase the numbers of the most metal-poor stars. Additionally, future analyses should incorporate non-LTE and $3 \mathrm{D}$ effects and processes.

We thank B. Edvardsson for computing additional MARCS model atmospheres and A. Chieffi, P. François, C. Kobayashi, A. Korn, and K. Venn for providing electronic data and/or helpful discussions. We thank the referee for helpful comments. D.Y., J.E.N., M.S.B., and M.A. gratefully acknowledge support from the Australian Research Council (grants DP03042613, DP0663562, DP0984924, and FL110100012) for studies of the Galaxy's most metal-poor stars and ultra-faint satellite systems. J.E.N. and D.Y. acknowledge financial support from the Access to Major Research Facilities Program, under the International Science Linkages Program of the Australian Federal Government. Australian access to the Magellan Telescopes was supported through the Major National Research Facilities program. Observations with the Keck Telescope were made under Gemini exchange time programs GN-2007B-C-20 and GN-2008A-C6. N.C. acknowledges financial support for this work through the Global Networks program of Universität Heidelberg and Sonderforschungsbereich SFB 881 "The Milky Way System" (subproject A4) of the German Research Foundation (DFG). T.C.B. acknowledges partial funding of this work from grants PHY 02-16783 and PHY 08-22648: Physics Frontier Center/ Joint Institute for Nuclear Astrophysics (JINA), awarded by the U.S. National Science Foundation. P.S.B. acknowledges support from the Royal Swedish Academy of Sciences and the Swedish Research Council. P.S.B. is a Royal Swedish Academy of Sciences Research Fellow supported by a grant from the Knut and Alice Wallenberg Foundation. The authors recognize and acknowledge the very significant cultural role and reverence that the summit of Mauna Kea has always had within the indigenous Hawaiian community. We are most fortunate to have the opportunity to conduct observations from this mountain. Finally, we are pleased to acknowledge support from the European Southern Observatory's Director's Discretionary Time Program.

Facilities: ATT (DBS), Keck:I (HIRES), Magellan:Clay (MIKE), VLT:Kueyen (UVES) 


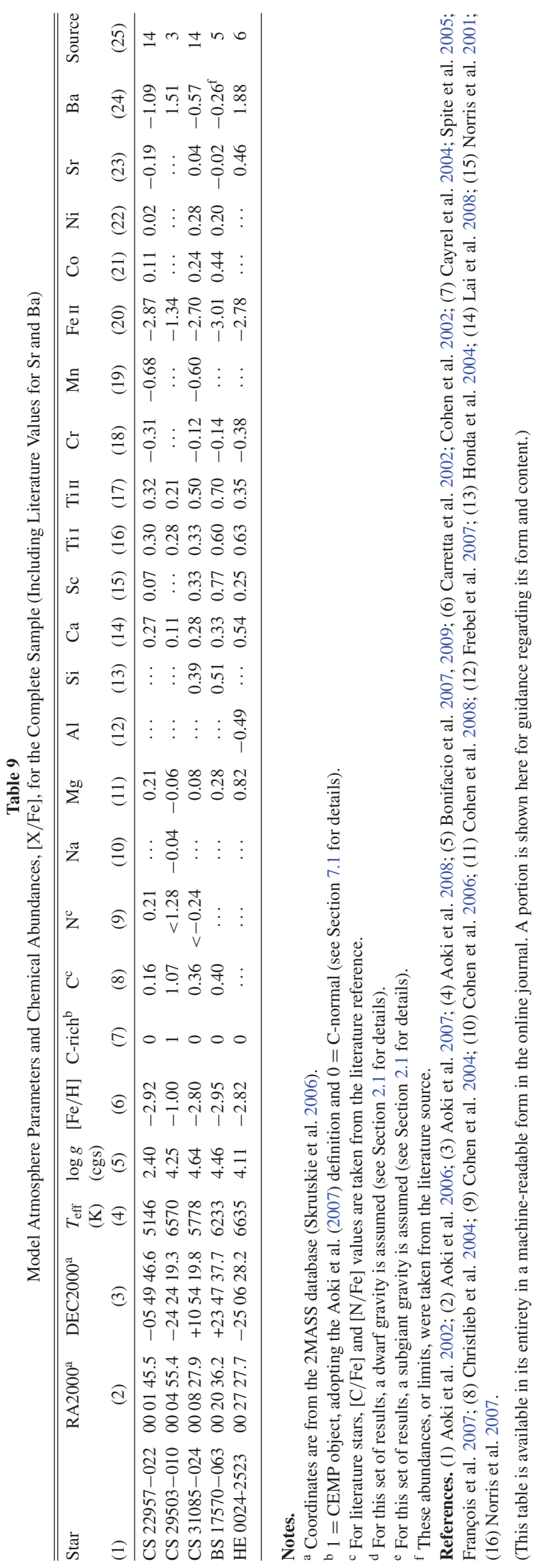




\section{APPENDIX}

\section{STELLAR PARAMETERS AND CHEMICAL ABUNDANCES FOR THE COMPLETE SAMPLE}

In Table 9, we provide the coordinates, stellar parameters, and abundance ratios for all of the program stars and literature stars presented in this work. Below is a description of the columns in the table.

(1) Star; (2) RA2000; (3) DEC2000; (4) effective temperature $\left(T_{\text {eff }}\right) ;(5)$ surface gravity $(\log g) ;(6)$ stellar metallicity $[\mathrm{Fe} /$ $\mathrm{H}]_{\text {derived }}$; (7) CEMP (0= no, $1=$ yes); (8) $[\mathrm{C} / \mathrm{Fe}]$; (9) $[\mathrm{N} / \mathrm{Fe}]$; (10) $[\mathrm{Na} / \mathrm{Fe}]$; (11) $[\mathrm{Mg} / \mathrm{Fe}]$; (12) $[\mathrm{Al} / \mathrm{Fe}]$; (13) $[\mathrm{Si} / \mathrm{Fe}]$; (14) $[\mathrm{Ca} / \mathrm{Fe}] ;$ (15) [Sc/Fe]; (16) [Ti I/Fe]; (17) [Ti II/Fe]; (18) [Cr/ $\mathrm{Fe}]$; (19) [Mn/Fe]; (20) [Fe II/H]; (21) [Co/Fe]; (22) [Ni/Fe]; (23) $[\mathrm{Sr} / \mathrm{Fe}]$; (24) $[\mathrm{Ba} / \mathrm{Fe}]$; (25) Source.

The $\mathrm{C}$ and $\mathrm{N}$ abundances for literature stars were taken directly from the literature sources. For the abundances of $\mathrm{Sr}$ and $\mathrm{Ba}$, in the cases in which the literature sources did not provide an equivalent width (i.e., they determined abundances using spectrum synthesis), we include (when available) the abundance measurements or limits. These literature values are flagged appropriately in the table.

\section{REFERENCES}

Abazajian, K. N., Adelman-McCarthy, J. K., Agüeros, M. A., et al. 2009, ApJS, 182,543

Allende Prieto, C., Barklem, P. S., Lambert, D. L., \& Cunha, K. 2004, A\&A, 420, 183

Alvarez, R., \& Plez, B. 1998, A\&A, 330, 1109

Alves-Brito, A., Meléndez, J., Asplund, M., Ramírez, I., \& Yong, D. 2010, A\&A, 513,35

Andrievsky, S. M., Spite, M., Korotin, S. A., et al. 2007, A\&A, 464, 1081 Andrievsky, S. M., Spite, M., Korotin, S. A., et al. 2008, A\&A, 481, 481 Andrievsky, S. M., Spite, F., Korotin, S. A., et al. 2011, A\&A, 530, A105

Aoki, W. 2010, in IAU Symp. 265, Chemical Abundances in the Universe: Connecting First Stars to Planets, ed. K. Cunha, M. Spite, \& B. Barbuy (Cambridge: Cambridge Univ. Press), 111

Aoki, W., Beers, T. C., Christlieb, N., et al. 2007, ApJ, 655, 492

Aoki, W., Beers, T. C., Sivarani, T., et al. 2008, ApJ, 678, 1351

Aoki, W., Frebel, A., Christlieb, N., et al. 2006, ApJ, 639, 897

Aoki, W., Honda, S., Beers, T. C., et al. 2005, ApJ, 632, 611

Aoki, W., Norris, J. E., Ryan, S. G., Beers, T. C., \& Ando, H. 2002, PASJ, 54,933

Aoki, W., Norris, J. E., Ryan, S. G., et al. 2004, ApJ, 608, 971

Arnone, E., Ryan, S. G., Argast, D., Norris, J. E., \& Beers, T. C. 2005, A\&A, 430, 507

Asplund, M. 2005, ARA\&A, 43, 481

Asplund, M., \& García Pérez, A. E. 2001, A\&A, 372, 601

Asplund, M., Grevesse, N., Sauval, A. J., \& Scott, P. 2009, ARA\&A, 47, 481

Barklem, P. S., Christlieb, N., Beers, T. C., et al. 2005, A\&A, 439, 129

Beers, T. C., \& Christlieb, N. 2005, ARA\&A, 43, 531

Bergemann, M. 2011, MNRAS, 413, 2184

Bergemann, M., \& Gehren, T. 2008, A\&A, 492, 823

Bergemann, M., Lind, K., Collet, R., Asplund, M., \& Magic, Z. 2012, MNRAS, 427, 27

Bergemann, M., Pickering, J. C., \& Gehren, T. 2010, MNRAS, 401, 1334

Bessell, M. S., \& Norris, J. 1984, ApJ, 285, 622

Bonifacio, P., Molaro, P., Sivarani, T., et al. 2007, A\&A, 462, 851

Bonifacio, P., Spite, M., Cayrel, R., et al. 2009, A\&A, 501, 519

Caffau, E., Bonifacio, P., François, P., et al. 2011, Natur, 477, 67

Carney, B. W., \& Peterson, R. C. 1981, ApJ, 245, 238

Carney, B. W., Wright, J. S., Sneden, C., et al. 1997, AJ, 114, 363

Carretta, E., Gratton, R., Cohen, J. G., Beers, T. C., \& Christlieb, N. 2002, AJ, 124,481

Casagrande, L., Ramírez, I., Meléndez, J., Bessell, M., \& Asplund, M. 2010, A\&A, 512, A54

Castelli, F., \& Kurucz, R. L. 2003, in IAU Symp. 210, Modelling of Stellar Atmospheres, ed. N. Piskunov, W. W. Weiss, \& D. F. Gray (San Francisco, CA: ASP), A20

Cayrel, R., Depagne, E., Spite, M., et al. 2004, A\&A, 416, 1117

Chamberlain, J. W., \& Aller, L. H. 1951, ApJ, 114, 52
Chieffi, A., Limongi, M., \& Straniero, O. 2000, in The Evolution of the Milky Way: Stars versus Clusters, ed. F. Matteucci \& F. Giovannelli (Dordrecht: Kluwer), 417

Christlieb, N., Bessell, M. S., Beers, T. C., et al. 2002, Natur, 419, 904

Christlieb, N., Gustafsson, B., Korn, A. J., et al. 2004, ApJ, 603, 708

Cleveland, W. S. 1979, J. Am. Stat. Assoc., 74, 829

Cohen, J. G., Christlieb, N., Beers, T. C., Gratton, R., \& Carretta, E. 2002, AJ, 124,470

Cohen, J. G., Christlieb, N., McWilliam, A., et al. 2004, ApJ, 612, 1107

Cohen, J. G., Christlieb, N., McWilliam, A., et al. 2008, ApJ, 672, 320

Cohen, J. G., \& Huang, W. 2010, ApJ, 719, 931

Cohen, J. G., McWilliam, A., Christlieb, N., et al. 2007, ApJL, 659, 161

Cohen, J. G., McWilliam, A., Shectman, S., et al. 2006, AJ, 132, 137

Collet, R., Hayek, W., Asplund, M., et al. 2011, A\&A, 528, A32

Cristallo, S., Piersanti, L., Straniero, O., et al. 2011, ApJS, 197, 17

Demarque, P., Woo, J., Kim, Y., \& Yi, S. K. 2004, ApJS, 155, 667

Dobrovolskas, V., Kučinskas, A., Andrievsky, S. M., et al. 2012, A\&A, 540, A 128

François, P., Depagne, E., Hill, V., et al. 2007, A\&A, 476, 935

Frebel, A. 2010, AN, 331, 474

Frebel, A., Aoki, W., Christlieb, N., et al. 2005, Natur, 434, 871

Frebel, A., \& Norris, J. E. 2011, arXiv:1102.1748

Frebel, A., Norris, J. E., Aoki, W., et al. 2007, ApJ, 658, 534

Fulbright, J. P. 2002, AJ, 123, 404

Griffin, R., Gustafsson, B., Vieira, T., \& Griffin, R. 1982, MNRAS, 198, 637

Gustafsson, B., Bell, R. A., Eriksson, K., \& Nordlund, A. 1975, A\&A, 42, 407

Gustafsson, B., Edvardsson, B., Eriksson, K., et al. 2008, A\&A, 486, 951

Heger, A., \& Woosley, S. E. 2010, ApJ, 724, 341

Hollek, J. K., Frebel, A., Roederer, I. U., et al. 2011, ApJ, 742, 54

Honda, S., Aoki, W., Ando, H., et al. 2004, ApJS, 152, 113

Hosford, A., García Pérez, A. E., Collet, R., et al. 2010, A\&A, 511, A47

Iben, I., Jr. 1964, ApJ, 140, 1631

Ito, H., Aoki, W., Honda, S., \& Beers, T. C. 2009, ApJL, 698, 37

Ivans, I. I., Sneden, C., James, C. R., et al. 2003, ApJ, 592, 906

Johnson, J. A. 2002, ApJS, 139, 219

Johnson, J. A., Herwig, F., Beers, T. C., \& Christlieb, N. 2007, ApJ, 658, 1203

Karakas, A., \& Lattanzio, J. C. 2007, PASA, 24, 103

Karakas, A. I. 2010, MNRAS, 403, 1413

Karlsson, T., \& Gustafsson, B. 2005, A\&A, 436, 879

Keller, S. C., Schmidt, B. P., Bessell, M. S., et al. 2007, PASA, 24, 1

Kobayashi, C., Izutani, N., Karakas, A. I., et al. 2011a, ApJL, 739, 57

Kobayashi, C., Karakas, A. I., \& Umeda, H. 2011b, MNRAS, 414, 3231

Kobayashi, C., \& Nakasato, N. 2011, ApJ, 729, 16

Kobayashi, C., Umeda, H., Nomoto, K., Tominaga, N., \& Ohkubo, T. 2006, ApJ, 653,1145

Korn, A. J. 2010, in Proc. 11th Symp. Nuclei in the Cosmos, Published online at http://pos.sissa.it/cgi-bin/reader/conf.cgi?confid=100, id.7

Korn, A. J., Grundahl, F., Richard, O., et al. 2007, ApJ, 671, 402

Kurucz, R., \& Bell, B. 1995, Atomic Line Data, Kurucz CD-ROM No. 23 (Cambridge, MA: Smithsonian Astrophysical Observatory), 23

Lai, D. K., Bolte, M., Johnson, J. A., et al. 2008, ApJ, 681, 1524

Lai, D. K., Rockosi, C. M., Bolte, M., et al. 2009, ApJL, 697, 63

Li, H. N., Christlieb, N., Schörck, T., et al. 2010, A\&A, 521, A10

Limongi, M., \& Chieffi, A. 2012, ApJS, 199, 38

Limongi, M., Chieffi, A., \& Bonifacio, P. 2003, ApJL, 594, 123

Lind, K., Asplund, M., \& Barklem, P. S. 2009a, A\&A, 503, 541

Lind, K., Asplund, M., Barklem, P. S., \& Belyaev, A. K. 2011, A\&A, 528, A103

Lind, K., Bergemann, M., \& Asplund, M. 2012, MNRAS, 427, 50

Lind, K., Korn, A. J., Barklem, P. S., \& Grundahl, F. 2008, A\&A, 490, 777

Lind, K., Primas, F., Charbonnel, C., Grundahl, F., \& Asplund, M. 2009b, A\&A, 503, 545

Lucatello, S., Tsangarides, S., Beers, T. C., et al. 2005, ApJ, 625, 825

Lugaro, M., Karakas, A. I., Stancliffe, R. J., \& Rijs, C. 2012, ApJ, 747, 2

Mashonkina, L., Gehren, T., Shi, J.-R., Korn, A. J., \& Grupp, F. 2011, A\&A, 528, A87

Masseron, T., Johnson, J. A., Plez, B., et al. 2010, A\&A, 509, 93

McWilliam, A. 1998, AJ, 115, 1640

McWilliam, A., Preston, G. W., Sneden, C., \& Searle, L. 1995, AJ, 109, 2757

Meléndez, J., Asplund, M., Alves-Brito, A., et al. 2008, A\&A, 484, L21

Meynet, G., Ekström, S., \& Maeder, A. 2006, A\&A, 447, 623

Nissen, P. E., Hoeg, E., \& Schuster, W. J. 1997, in Hipparcos: Venice '97, ed. R. M. Bonnet, E. Høg, P. L. Bernacca, L. Emiliani, A. Blaauw, C. Turon, J. Kovalevsky, L. Lindegren, H. Hassan, M. Bouffard, B. Strim, D. Heger,

M. A. C. Perryman, \& L. Woltjer (ESP-402; Noordwijk: ESA), 225

Nissen, P. E., \& Schuster, W. J. 1997, A\&A, 326, 751

Nissen, P. E., \& Schuster, W. J. 2010, A\&A, 511, L10

Norris, J. E., Bessell, M. S., Yong, D., et al. 2013a, ApJ, 762, 25 (Paper I) 
Norris, J. E., Christlieb, N., Korn, A. J., et al. 2007, ApJ, 670, 774

Norris, J. E., Gilmore, G., Wyse, R. F. G., Yong, D., \& Frebel, A. 2010a, ApJL, 722,104

Norris, J. E., Ryan, S. G., \& Beers, T. C. 1997, ApJL, 489, 169

Norris, J. E., Ryan, S. G., \& Beers, T. C. 2001, ApJ, 561, 1034

Norris, J. E., Yong, D., Bessell, M. S., et al. 2013b, ApJ, 762, 28 (Paper IV)

Norris, J. E., Yong, D., Gilmore, G., \& Wyse, R. F. G. 2010b, ApJ, 711, 350

Plez, B., \& Cohen, J. G. 2005, A\&A, 434, 1117

Pols, O. R., Izzard, R. G., Stancliffe, R. J., \& Glebbeek, E. 2012, A\&A, 547, A76

Ramírez, I., \& Meléndez, J. 2005, ApJ, 626, 465

Reddy, B. E., Tomkin, J., Lambert, D. L., \& Allende Prieto, C. 2003, MNRAS, 340, 304

Richard, O., Michaud, G., \& Richer, J. 2002a, ApJ, 580, 1100

Richard, O., Michaud, G., Richer, J., et al. 2002b, ApJ, 568, 979

Roederer, I. U. 2009, AJ, 137, 272

Roederer, I. U., Cowan, J. J., Karakas, A. I., et al. 2010, ApJ, 724, 975

Ryan, S. G., Norris, J. E., \& Beers, T. C. 1996, ApJ, 471, 254

Sbordone, L., Bonifacio, P., Caffau, E., \& Ludwig, H.-G. 2012, in ASP Conf. Ser. 458, Galactic Archaeology: Near-Field Cosmology and the Formation of the Milky Way, ed. W. Aoki, M. Ishigaki, T. Suda, T. Tsujimoto, \& N. Arimoto (San Francisco, CA: ASP), 69
Schörck, T., Christlieb, N., Cohen, J. G., et al. 2009, A\&A, 507, 817 Skrutskie, M. F., Cutri, R. M., Stiening, R., et al. 2006, AJ, 131, 1163 Sneden, C. 1973, ApJ, 184, 839

Sobeck, J. S., Kraft, R. P., Sneden, C., et al. 2011, AJ, 141, 175

Spite, M., Andrievsky, S. M., Spite, F., et al. 2012, A\&A, 541, A143

Spite, M., Cayrel, R., Plez, B., et al. 2005, A\&A, 430, 655

Stephens, A., \& Boesgaard, A. M. 2002, AJ, 123, 1647

Suda, T., Katsuta, Y., Yamada, S., et al. 2008, PASJ, 60, 1159

Suda, T., Yamada, S., Katsuta, Y., et al. 2011, MNRAS, 412, 843

Taylor, J. 1997, Introduction to Error Analysis, the Study of Uncertainties in

Physical Measurements (2nd ed.; Mill Valley, CA: Univ. Science Books)

Thévenin, F., \& Idiart, T. P. 1999, ApJ, 521, 753

Tolstoy, E., Hill, V., \& Tosi, M. 2009, ARA\&A, 47, 37

Tominaga, N., Umeda, H., \& Nomoto, K. 2007, ApJ, 660, 516

Travaglio, C., Gallino, R., Arnone, E., et al. 2004, ApJ, 601, 864

Umeda, H., \& Nomoto, K. 2005, ApJ, 619, 427

Venn, K. A., Shetrone, M. D., Irwin, M. J., et al. 2012, ApJ, 751, 102

Wallerstein, G., Greenstein, J. L., Parker, R., Helfer, H. L., \& Aller, L. H. 1963, ApJ, 137, 280

Wisotzki, L., Koehler, T., Groote, D., \& Reimers, D. 1996, A\&AS, 115, 227

Yong, D., Norris, J. E., Bessell, M. S., et al. 2013, ApJ, 762, 27 (Paper III)

Zhao, G., Chen, Y.-Q., Shi, J.-R., et al. 2006, ChJAA, 6, 265 